\title{
Silver-Mediated Synthesis of Substituted Benzofuran- and Indole-Pyrroles via Sequential Reaction of ortho-Alkynylaromatics with Methylene Isocyanides
}

Jian-Quan Liu, ${ }^{+\ddagger}$ Xinyi Chen, ${ }^{\dagger}$ Andrey Shatskiy, ${ }^{\ddagger}$ Markus D. Kärkäs, ${ }^{*} \ddagger$ Xiang-Shan Wang ${ }^{*, \dagger}$

+ School of Chemistry and Materials Science, Jiangsu Key Laboratory of Green Synthesis for Functional Materials Jiangsu Normal University, Xuzhou, Jiangsu 221116, China

‡ Department of Chemistry, KTH Royal Institute of Technology, SE-100 44 Stockholm, Sweden

*E-mail:karkas@kth.se; xswang1974@yahoo.com 


\section{Table of Contents}

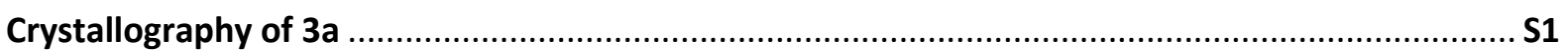

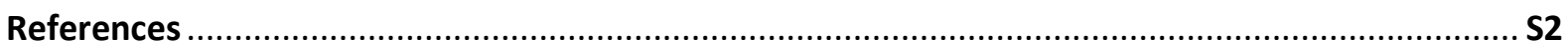

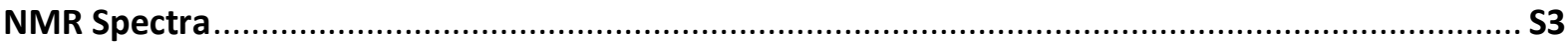

Compound 1a

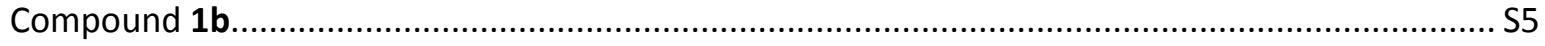

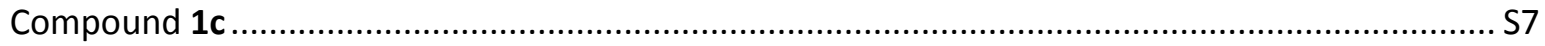

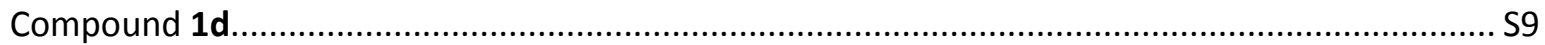

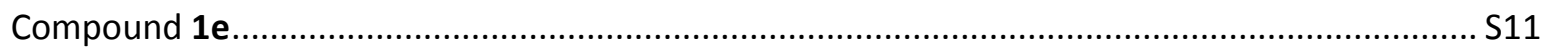

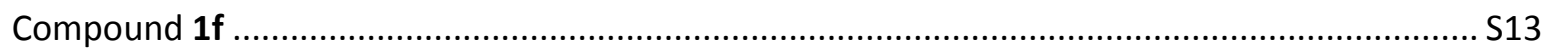

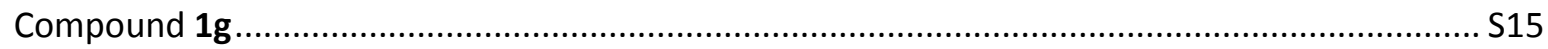

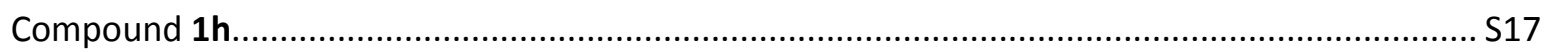

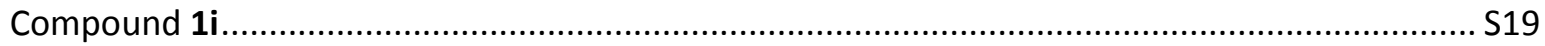

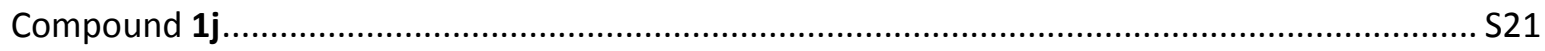

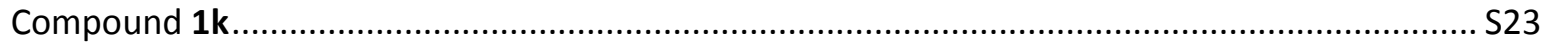

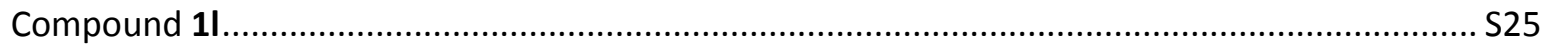

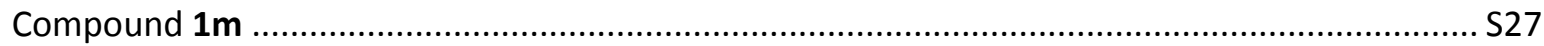

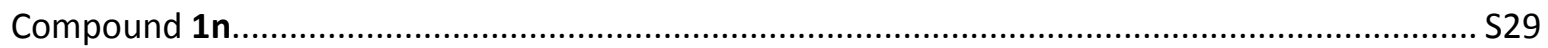

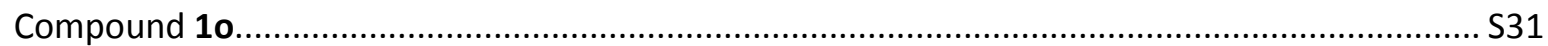

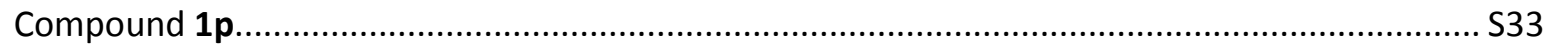

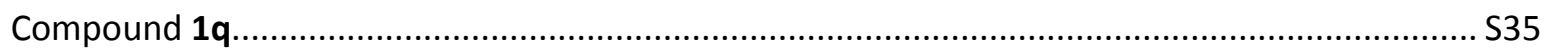

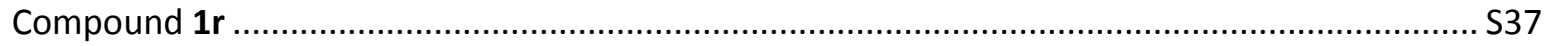

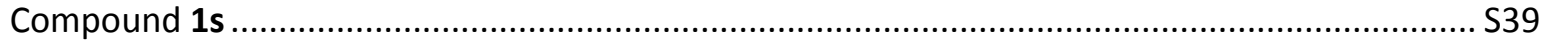

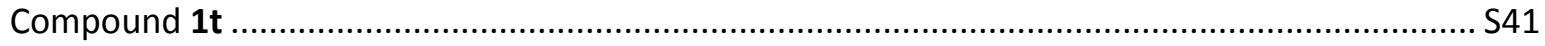

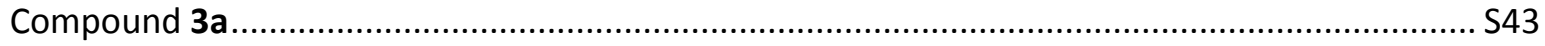

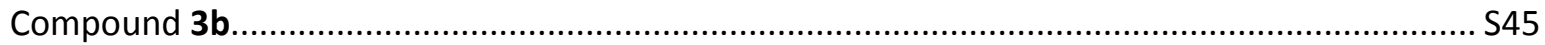

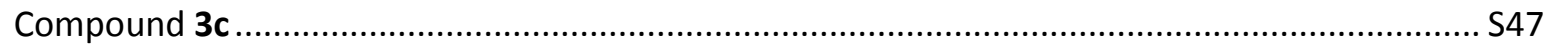

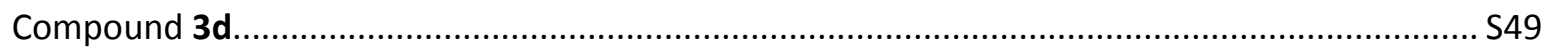

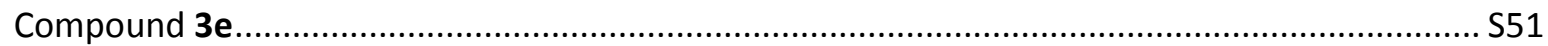

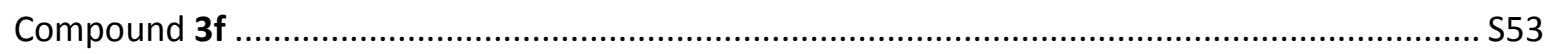

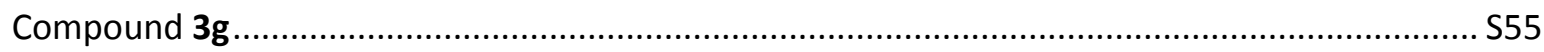

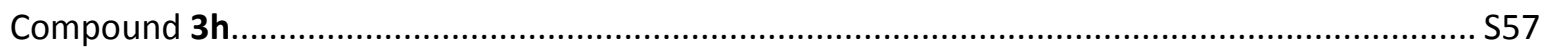

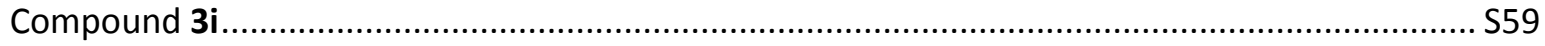




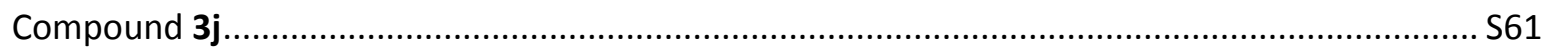

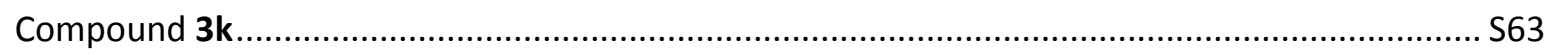

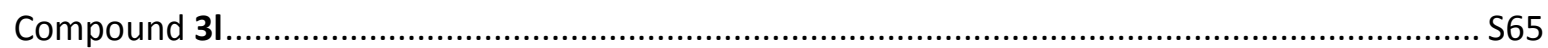

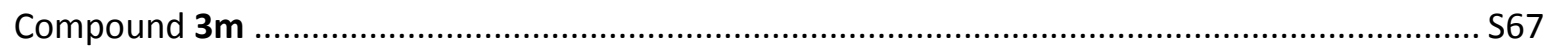

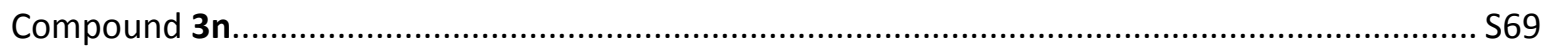

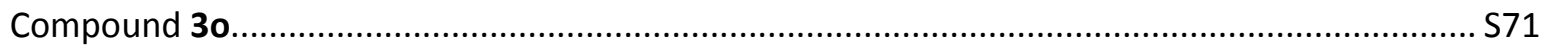

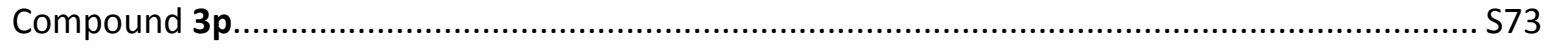

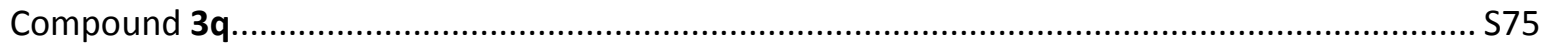

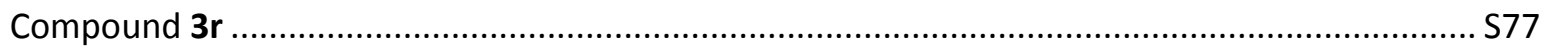

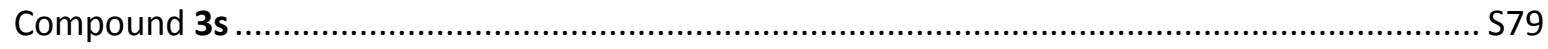

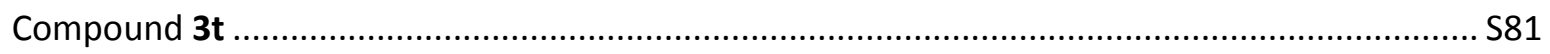

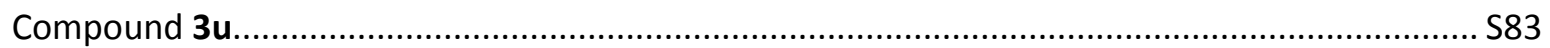

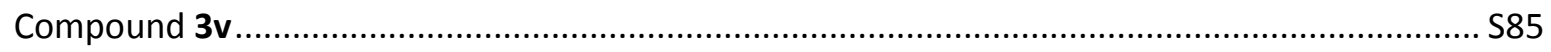

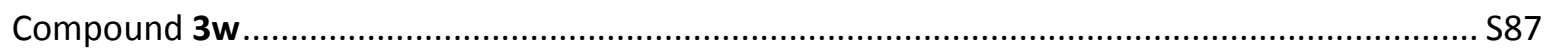

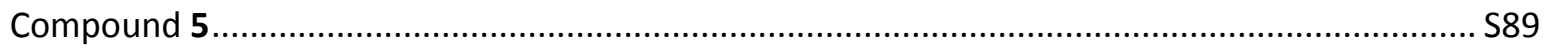

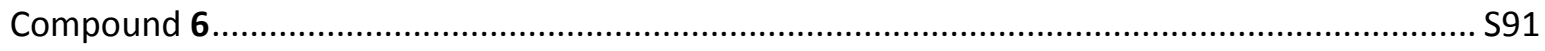

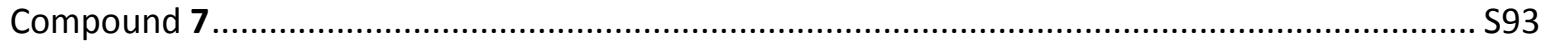

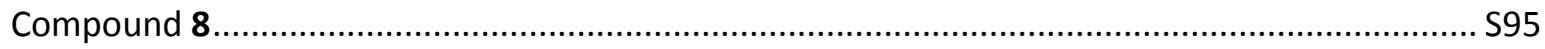




\section{Crystallography of 3a}

The structure of 3a was further established by X-ray diffraction. Single-crystals were obtained from a petroleum ether/ethyl acetate (3:1) solution. X-ray diffraction data for the reported compound was recorded at a temperature of 296(2) K on an Oxford Diffraction Gemini R Ultra diffractometer, using a $\omega$ scan technique with Mo-K $\alpha$ radiation $(\lambda=0.71073 \AA ̊)$. The structure was solved by Direct Method of SHELXS-97 and refined by full-matrix least-squares techniques using the SHELXL-97 program. ${ }^{1}$ Non-hydrogen atoms were refined with anisotropic temperature parameters, and hydrogen atoms of the ligands were refined as rigid groups. Basic information pertaining to crystal parameters and structure refinement are summarized in Table S1.

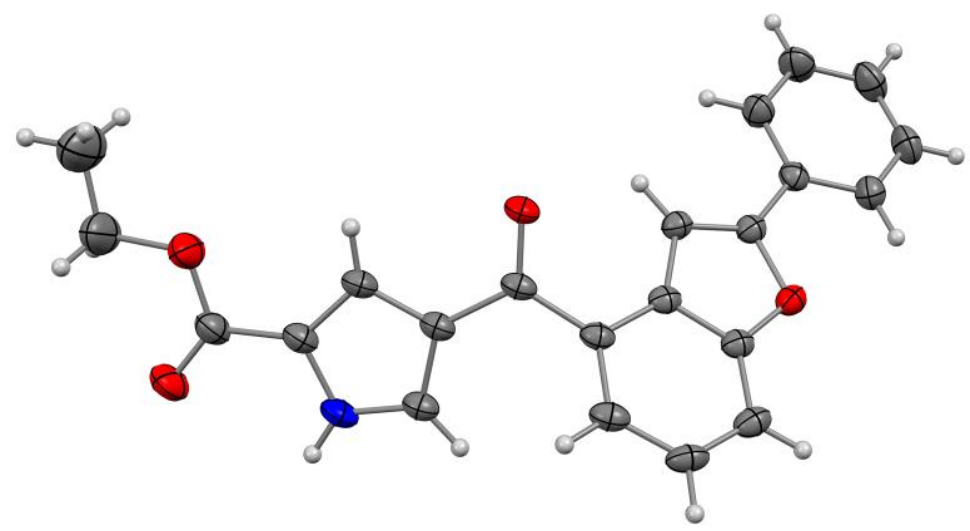

Table S1. Crystal data of 3a with 30\% probability levels and structure refinement.

\begin{tabular}{ll}
\hline Empirical formula & $\mathrm{C}_{22} \mathrm{H}_{17} \mathrm{NO}_{4}$ \\
Formula weight & 359.37 \\
Temperature & $296(2) \mathrm{K}$ \\
Wavelength & $0.71073 \AA$ \\
Crystal system & Orthorhombic \\
Space group & $\mathrm{Pna2}(1)$ \\
Unit cell dimensions & $\mathrm{a}=14.418(4) \AA$ \\
& $\mathrm{b}=18.761(6) \AA$ \\
& $\mathrm{c}=6.767(2) \AA$ \\
& alpha $=90 \mathrm{deg}$. \\
& beta $=90 \mathrm{deg}$. \\
& gamma $=90 \mathrm{deg}$. \\
Volume & $1830.3(9) \AA^{3}$ \\
Z & 4 \\
Calculated density & $1.304 \mathrm{Mg} / \mathrm{m}^{3}$ \\
Absorption coefficient & $1.242 \mathrm{~mm}$ \\
F(000) & 752 \\
Crystal size & $0.250 \times 0.250 \times 0.150 \mathrm{~mm}$ \\
\hline
\end{tabular}




\begin{tabular}{ll}
\hline Theta range for data collection & 3.03 to 25.02 deg. \\
Index ranges & $-17<=\mathrm{h}<=15,-22<=\mathrm{k}<=22,-8<=\mathrm{I}<=7$ \\
Reflections collected / unique & $10521 / 3093[\mathrm{R}(\mathrm{int})=0.0281]$ \\
Refinement method & Full-matrix least-squares on $\mathrm{F}^{2}$ \\
Data / restraints / parameters & $3093 / 2 / 248$ \\
Goodness-of-fit on $\mathrm{F}^{2}$ & 1.069 \\
Final $\mathrm{R}$ indices [I>2sigma(I)] & $\mathrm{R} 1=0.0416, \mathrm{WR} 2=0.0919$ \\
$\mathrm{R}$ indices (all data) & $\mathrm{R} 1=0.0519, \mathrm{wR} 2=0.0960$ \\
\hline
\end{tabular}

\section{References}

(1) (a) G. M. Sheldrick, SHELXS-97, Program for Solution of Crystal Structures, University of Gottingen, Germany, 1997. (b) G. M. Sheldrick, SHELXL-97, Program for Refinement of Crystal Structures, University of Gottingen, Germany, 1997. 
NMR Spectra

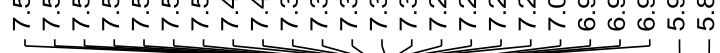

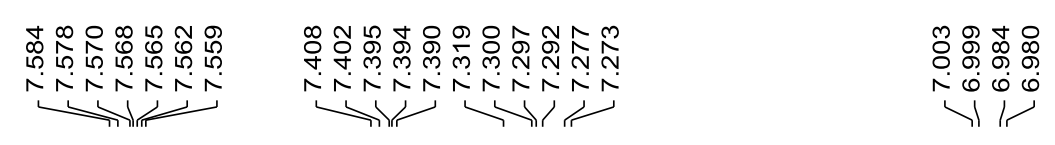
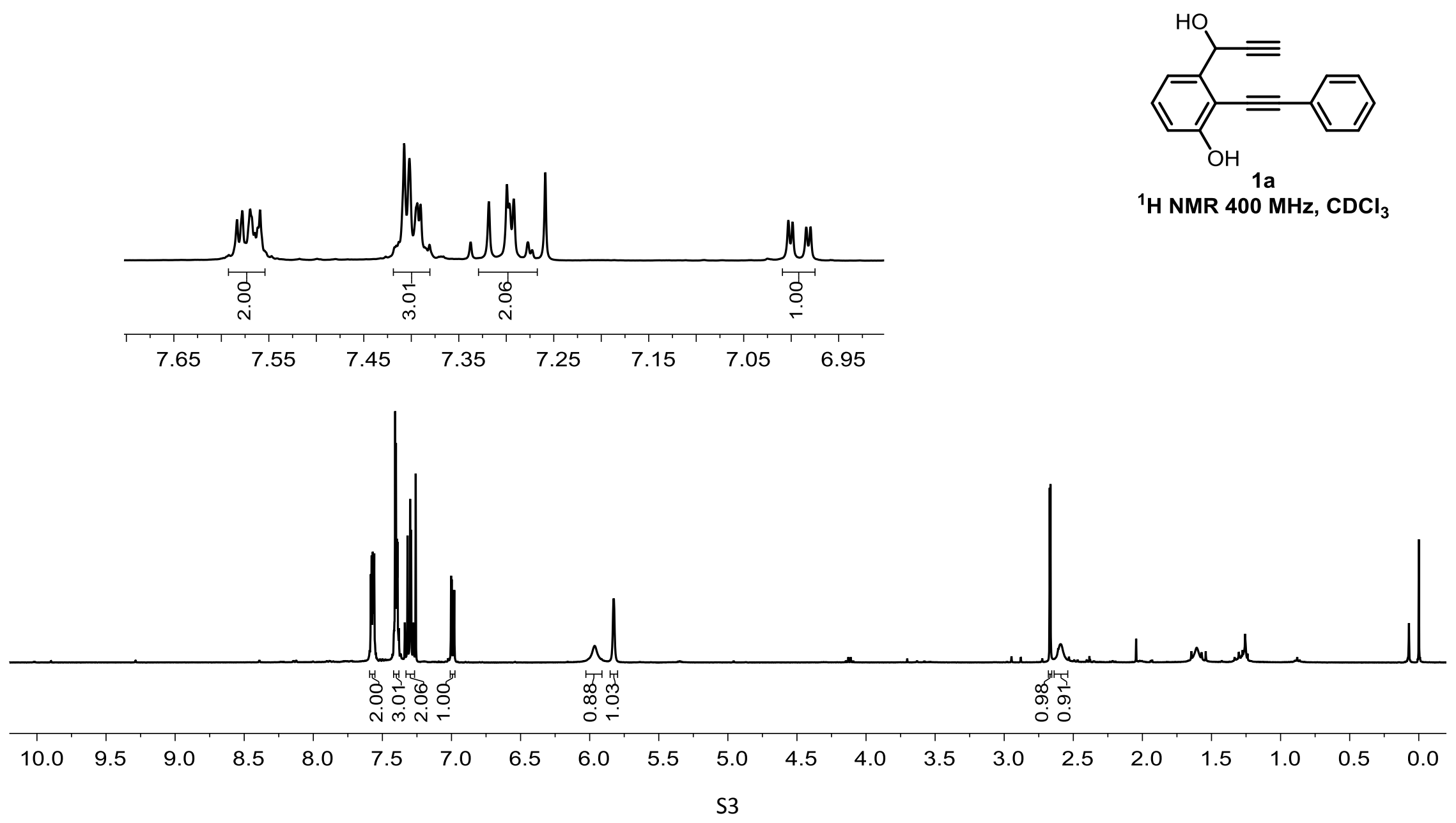

${ }^{1} \mathrm{H}$ NMR $400 \mathrm{MHz}, \mathrm{CDCl}_{3}$ 


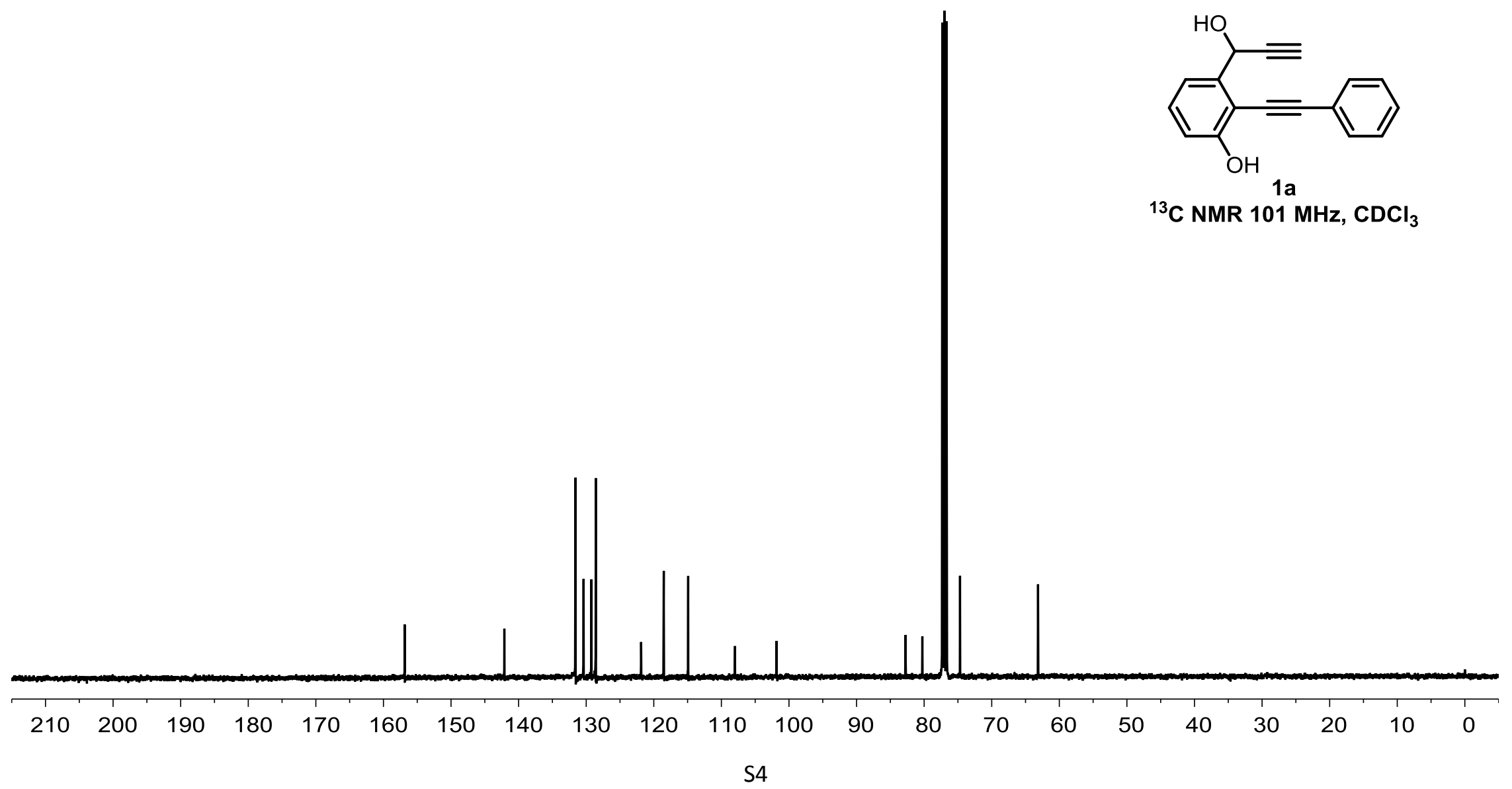




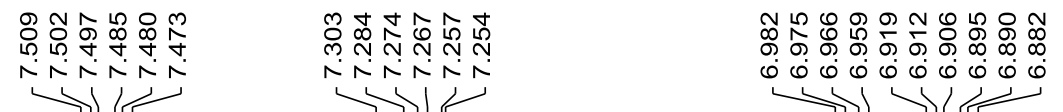

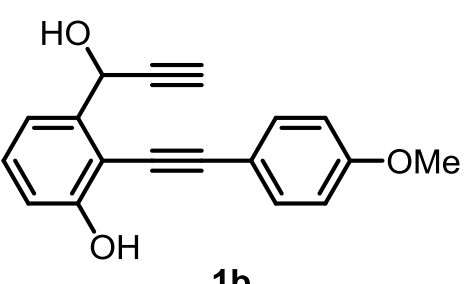

${ }^{1} \mathrm{H}$ NMR $400 \mathrm{MHz}, \mathrm{CDCl}_{3}$
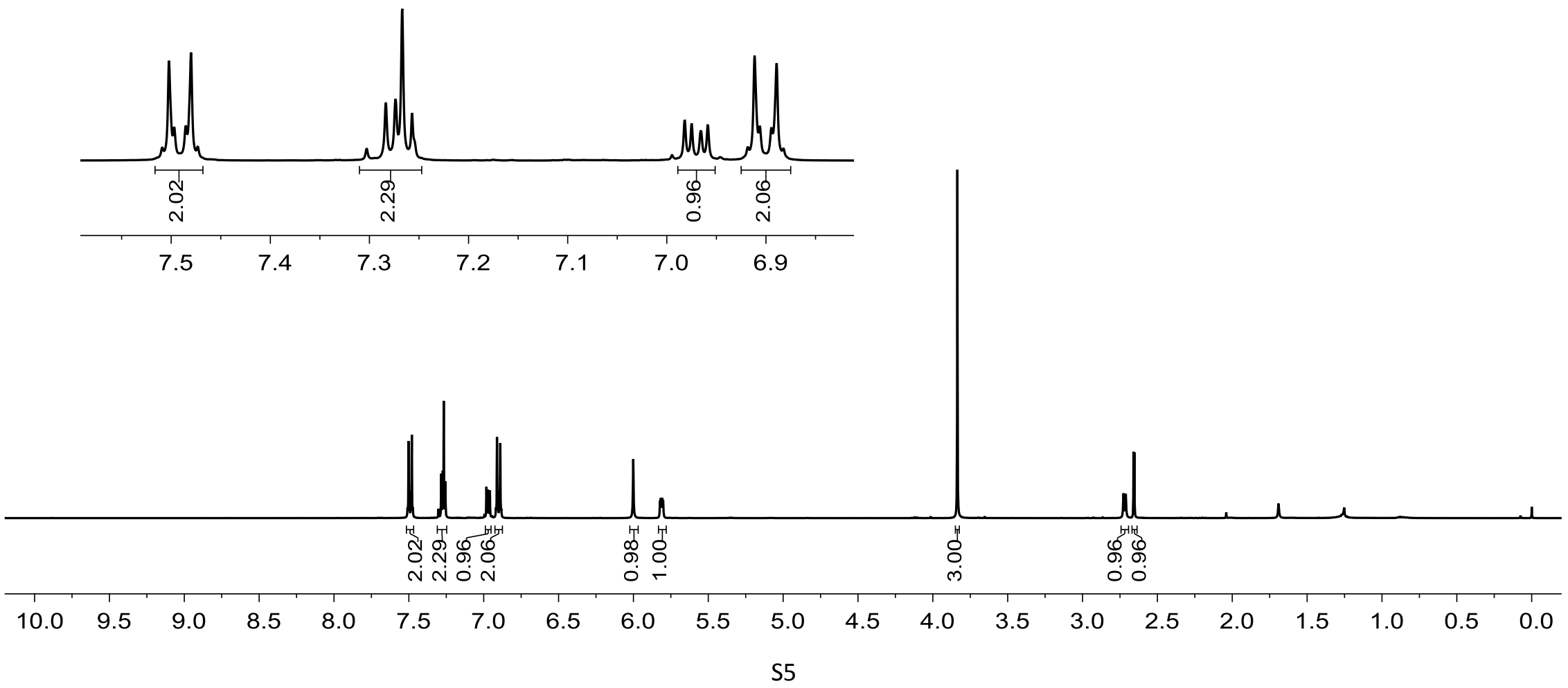


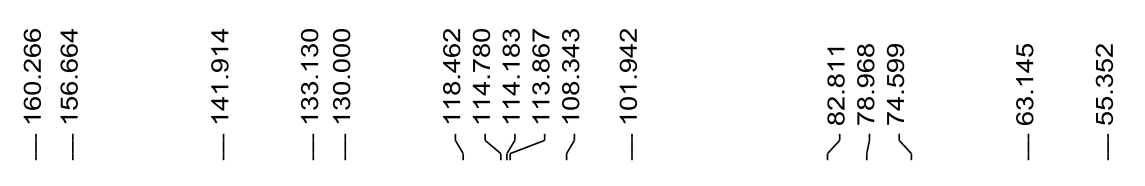
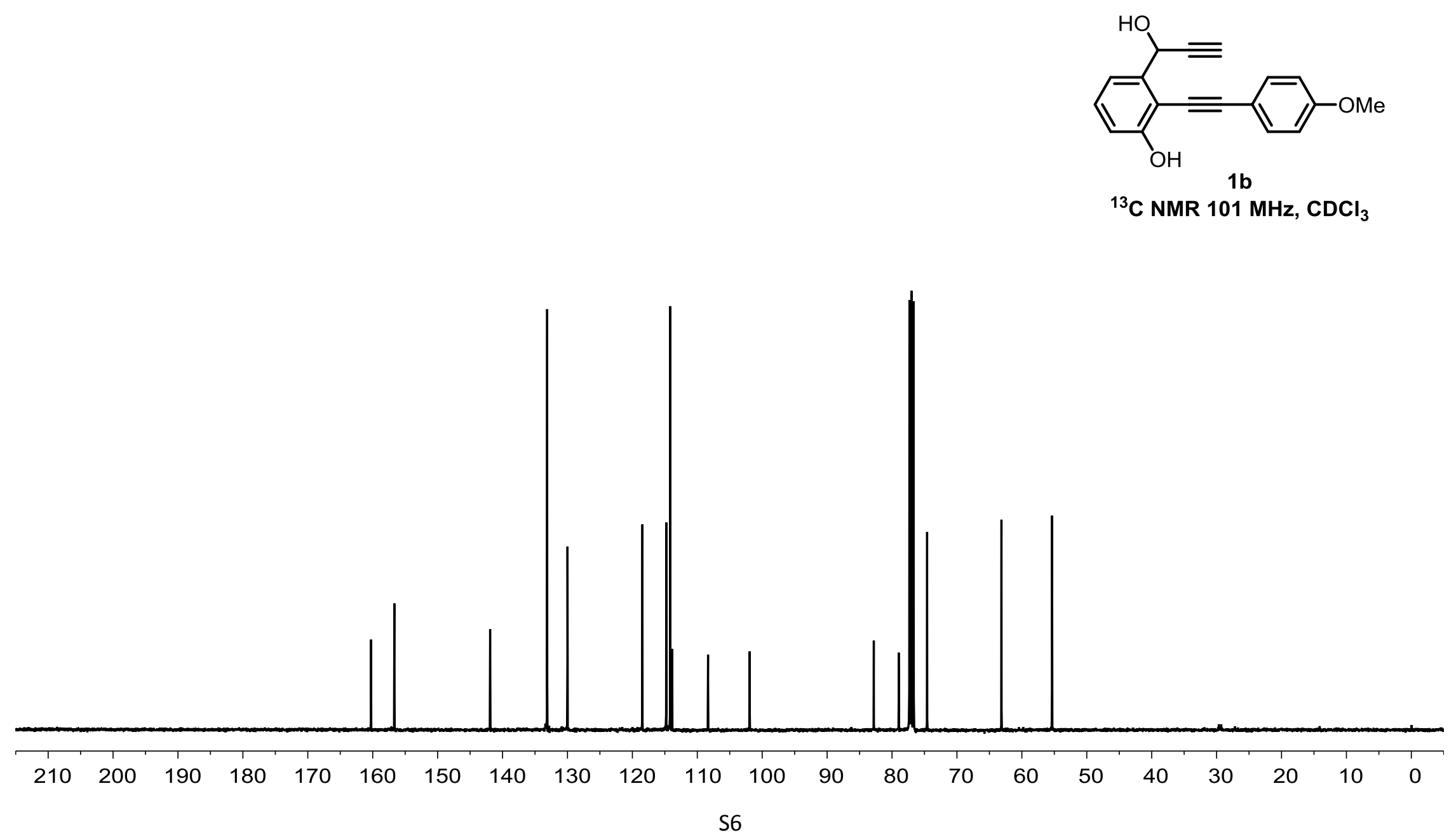


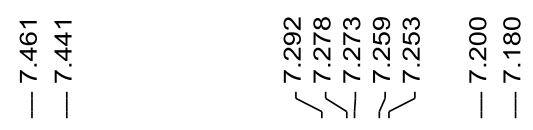

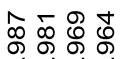

0

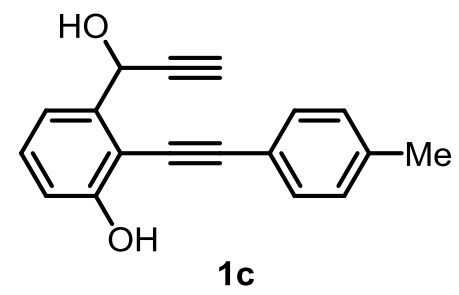

${ }^{1} \mathrm{H}$ NMR $400 \mathrm{MHz}^{\mathrm{CDCl}_{3}}$

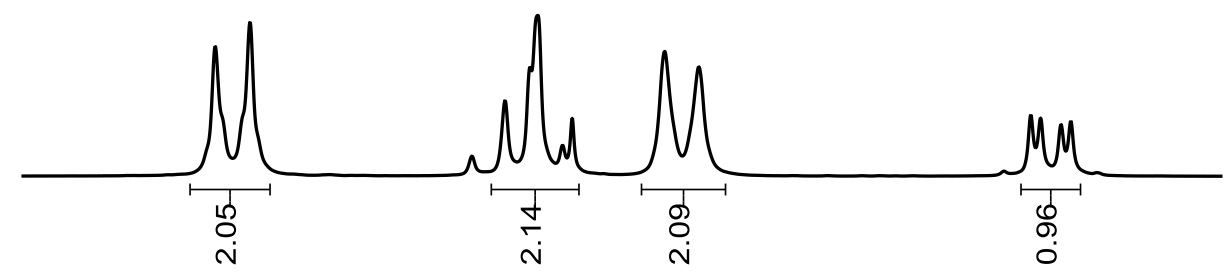

อ.

$7.557 .507 .457 .407 .357 .307 .257 .207 .157 .107 .05 \quad 7.006 .956 .90$

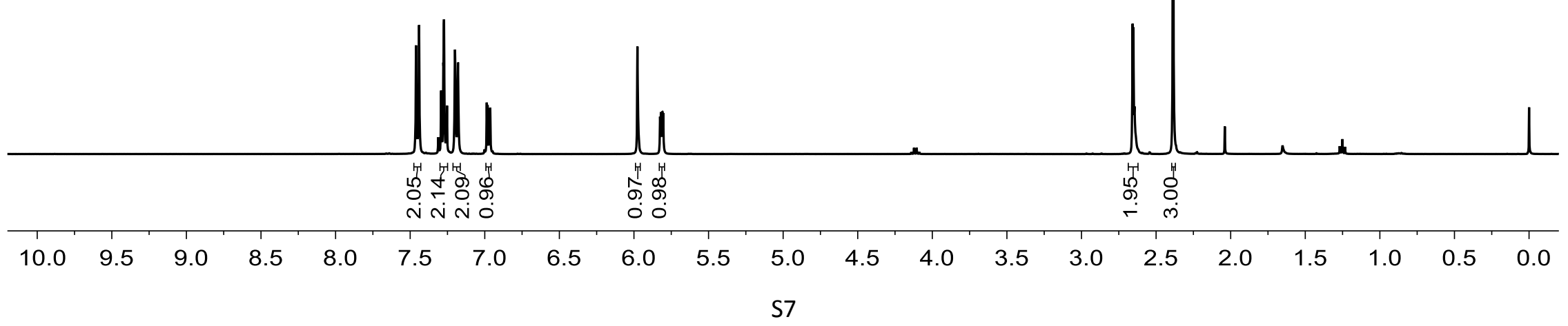




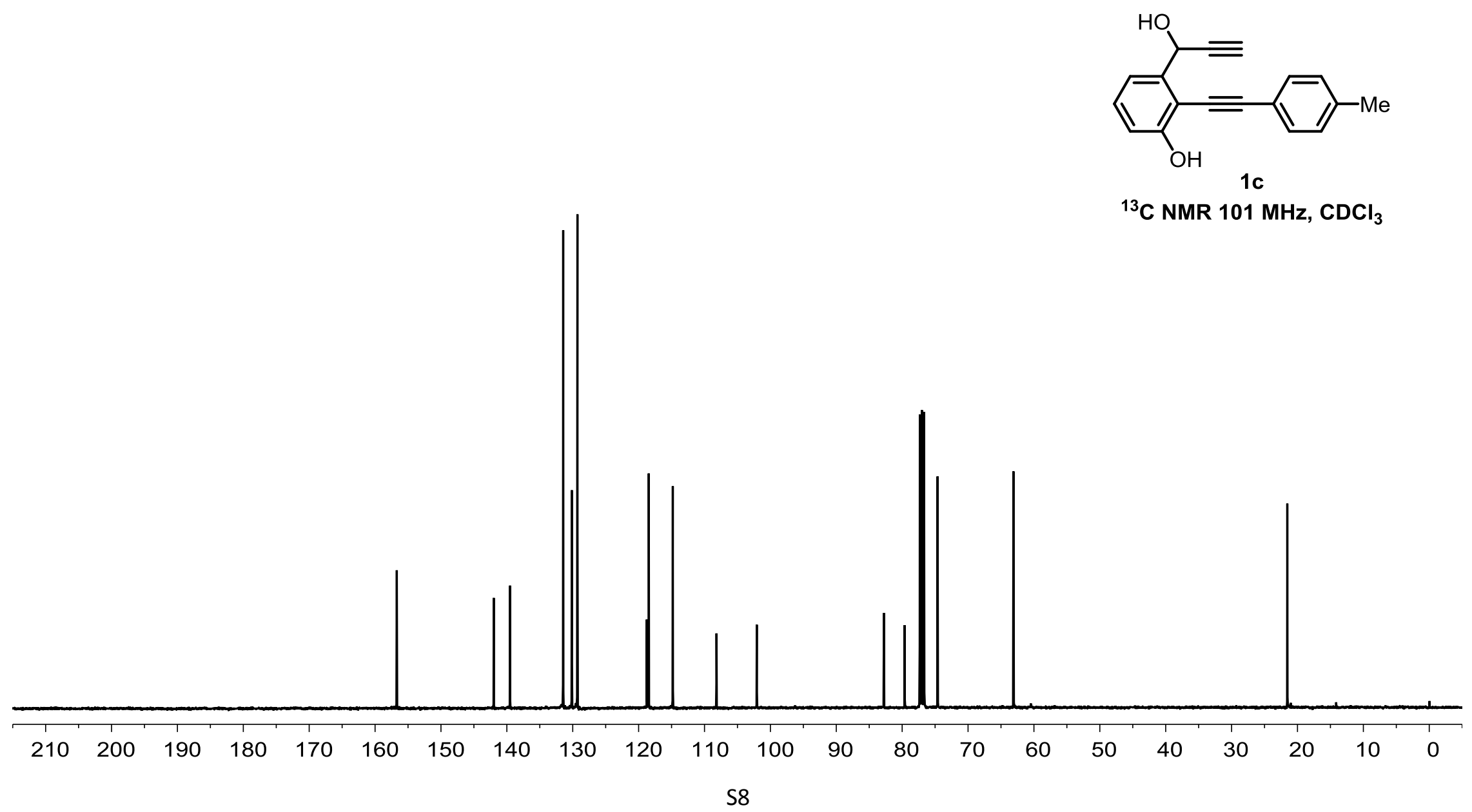




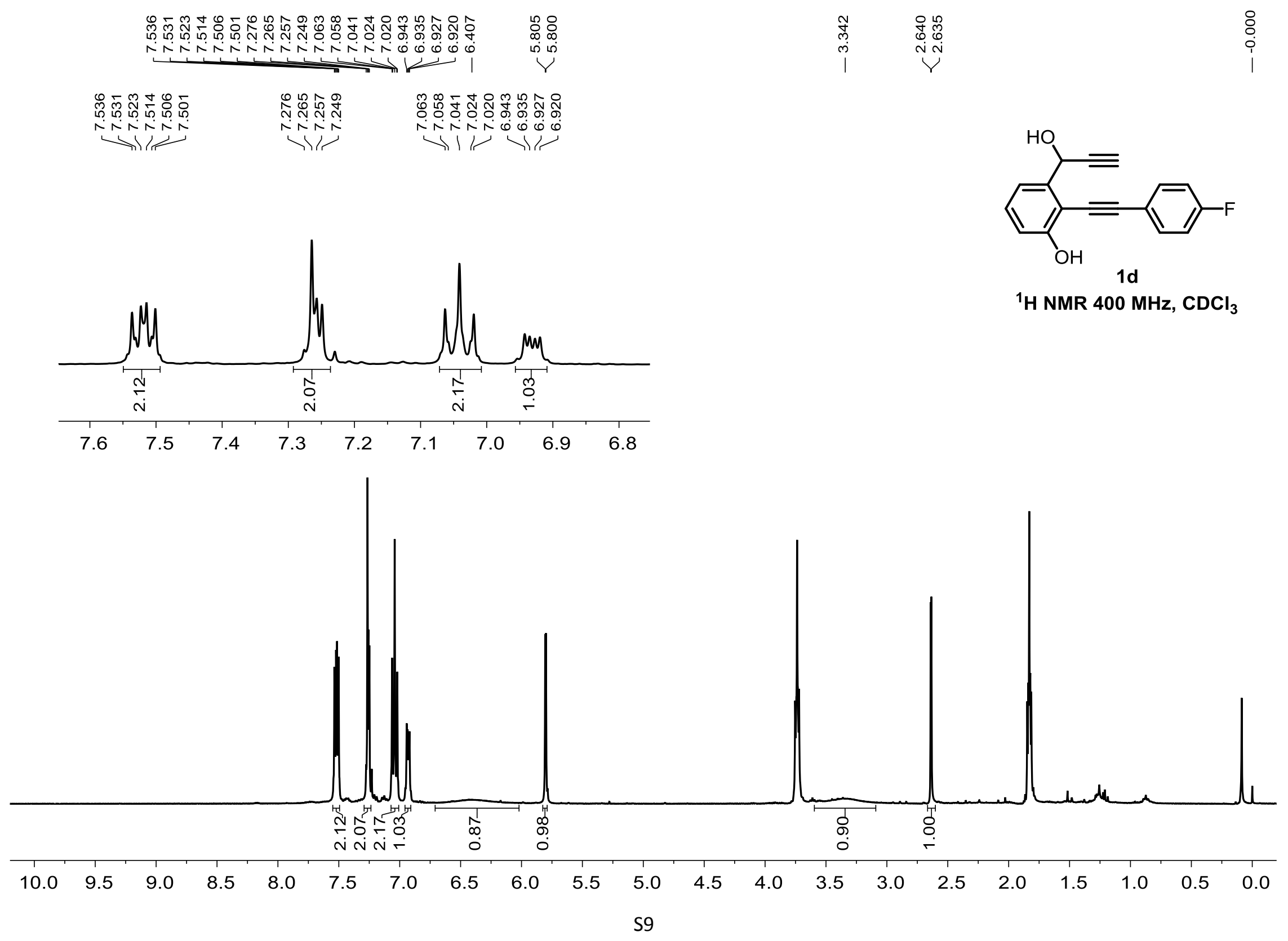




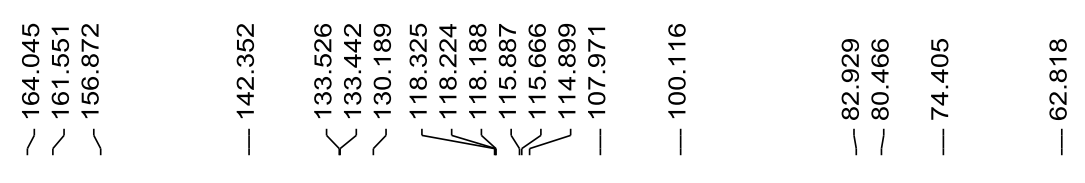

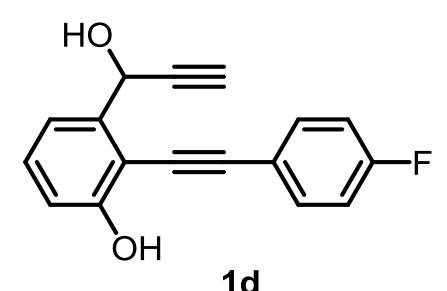

${ }^{13} \mathrm{C}$ NMR $101 \mathrm{MHz}, \mathrm{CDCl}_{3}$

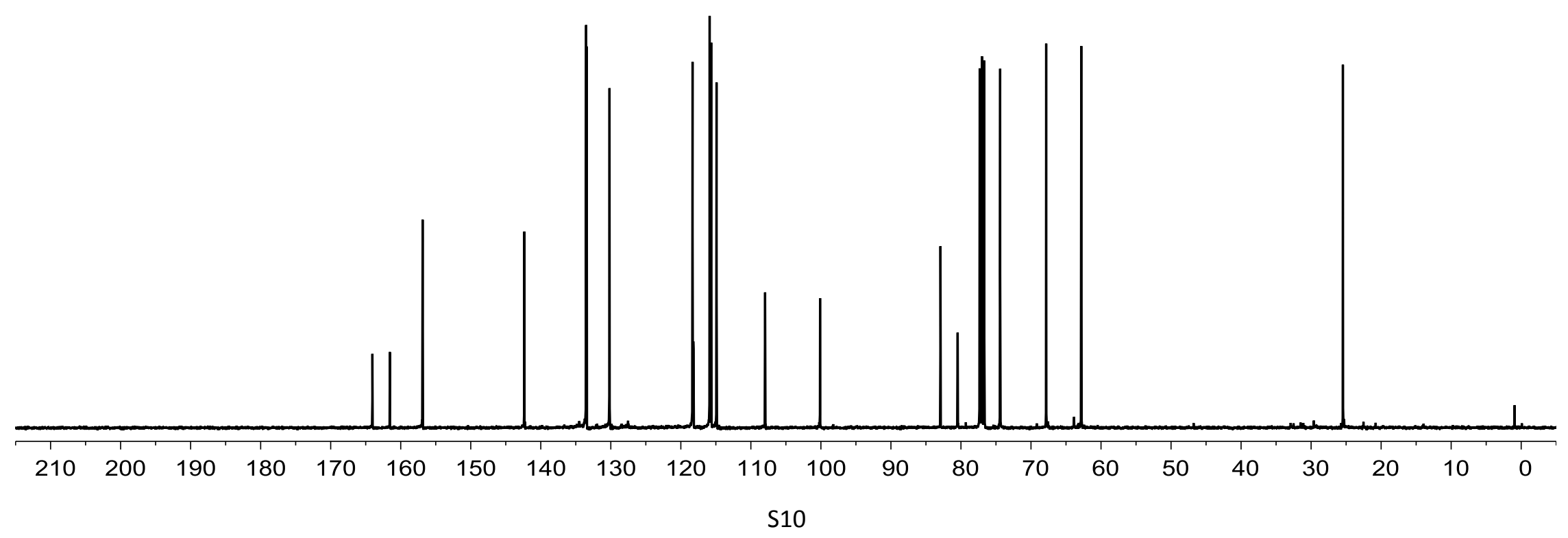



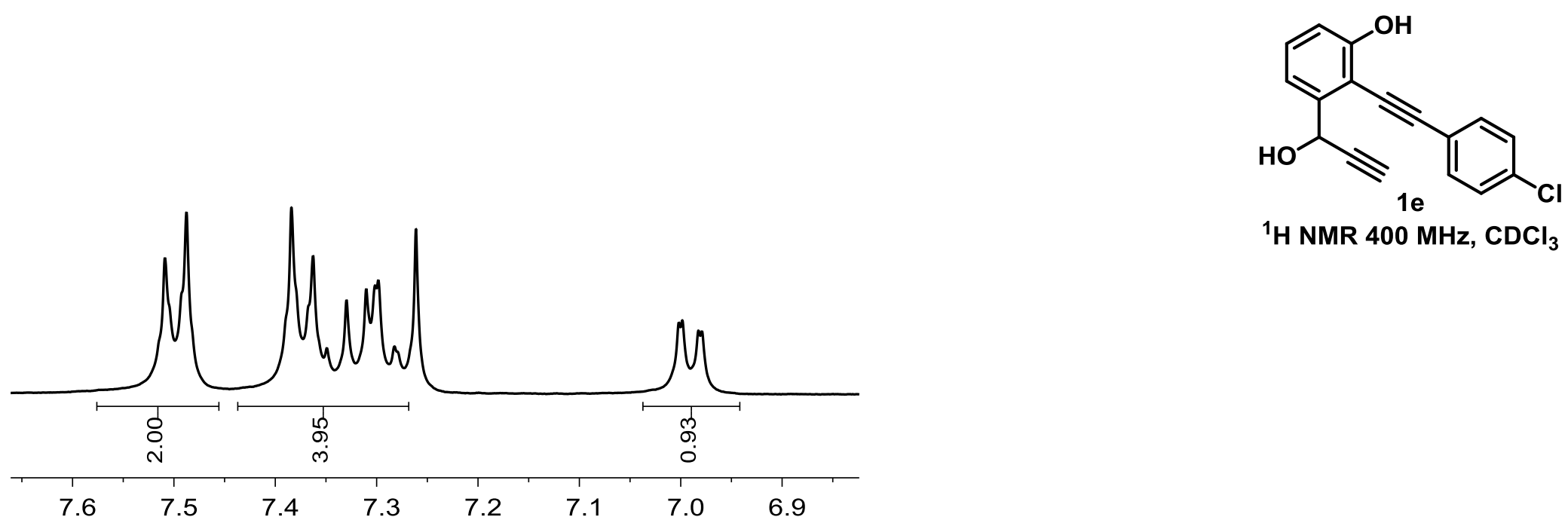

${ }^{1} \mathrm{H}$ NMR $400 \mathrm{MHz}, \mathrm{CDCl}_{3}$

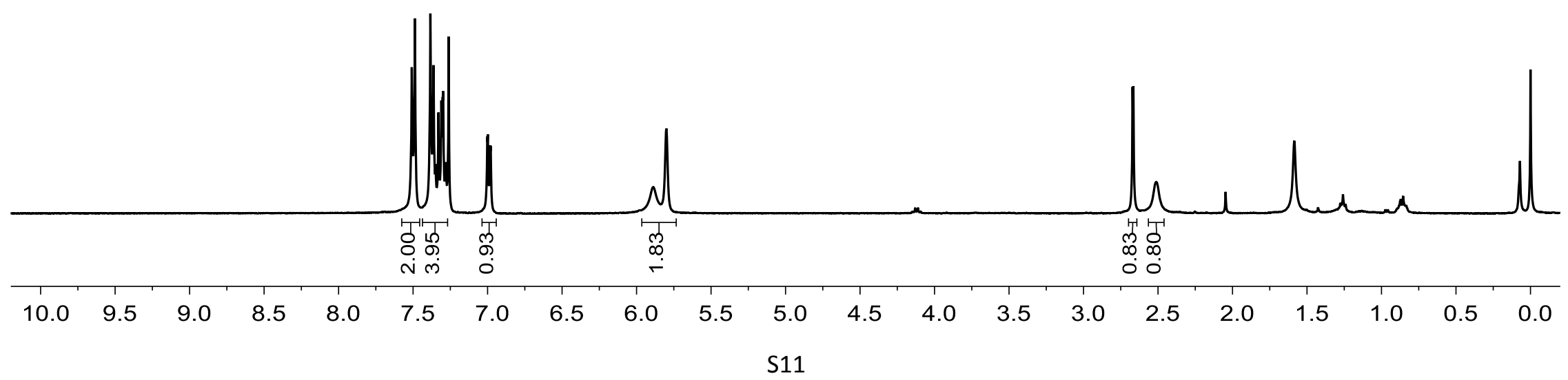




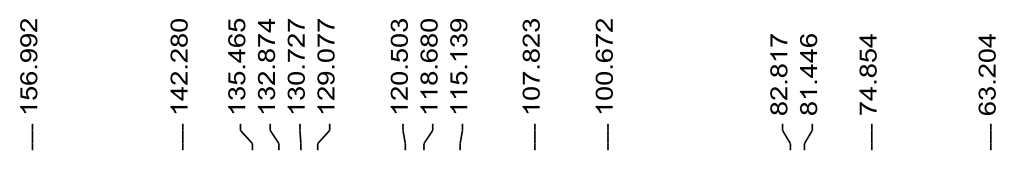
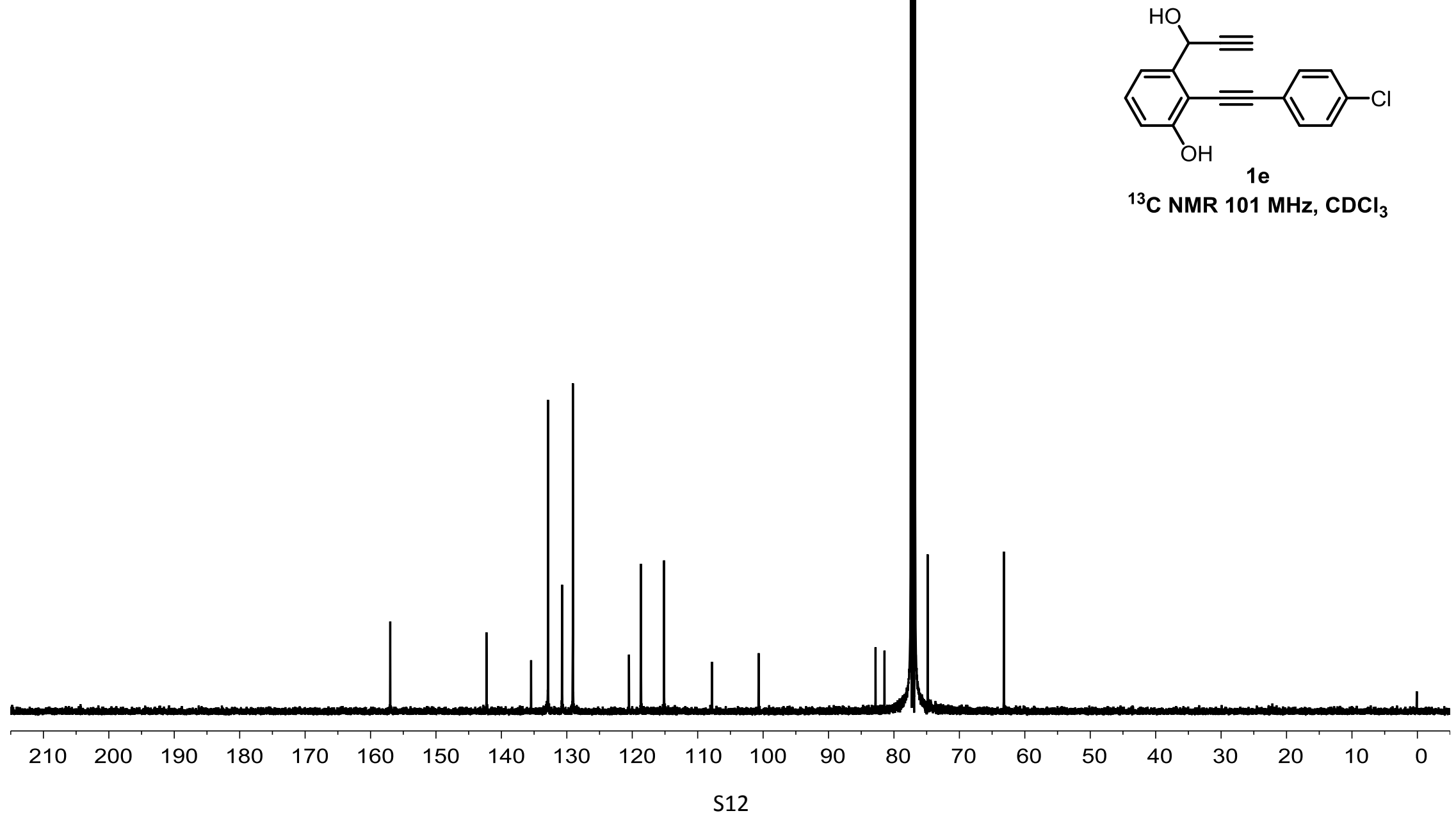


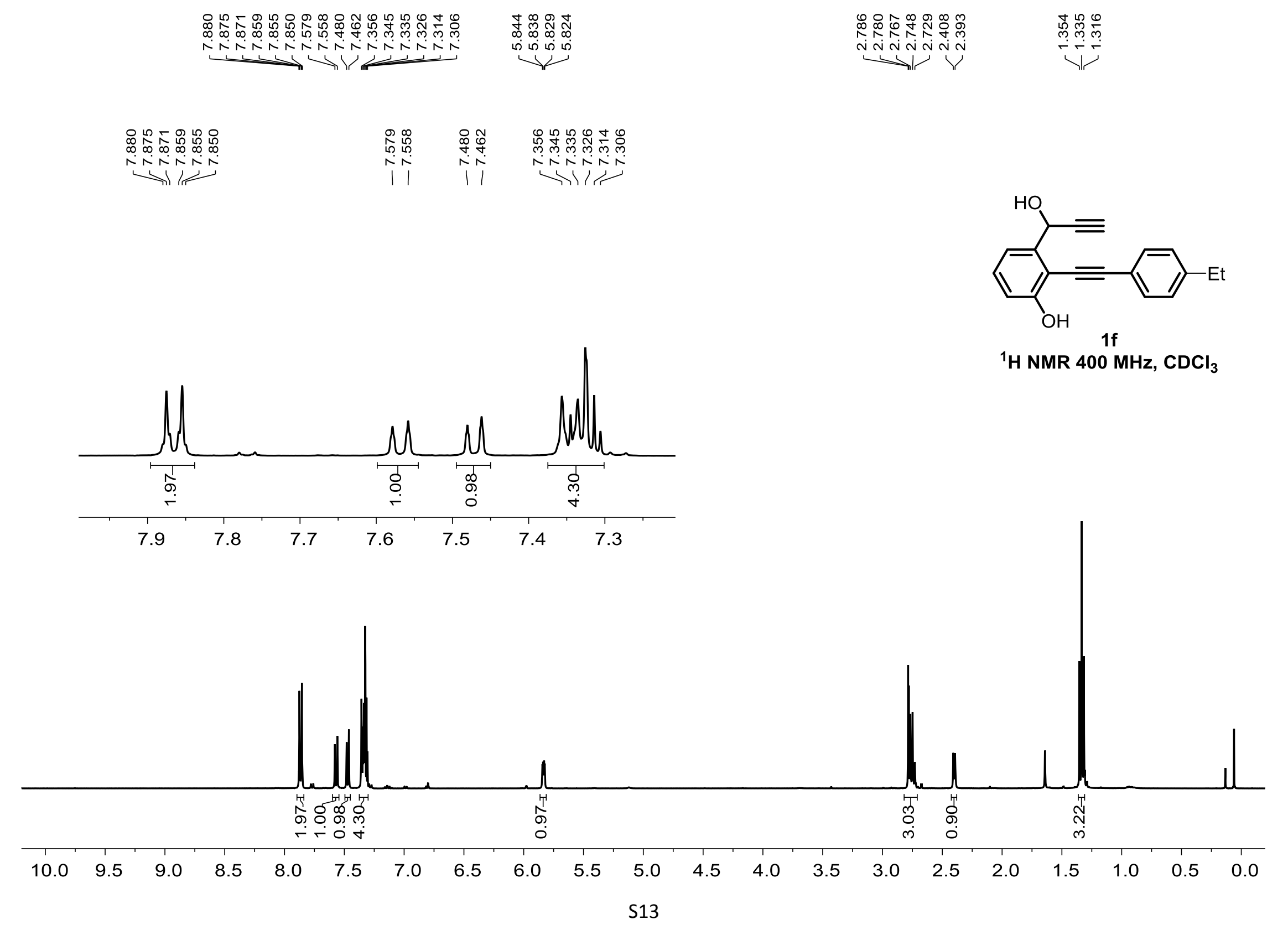




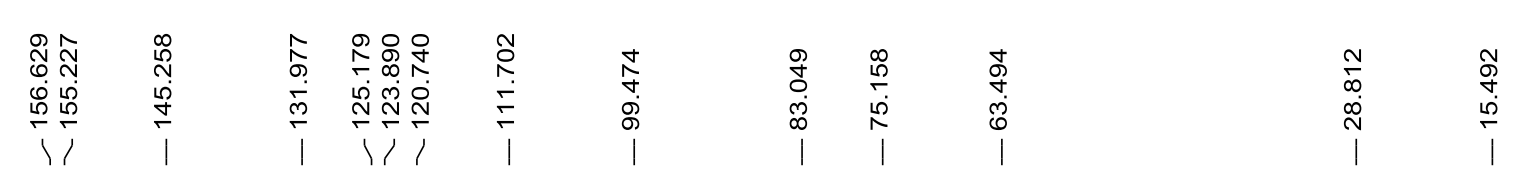

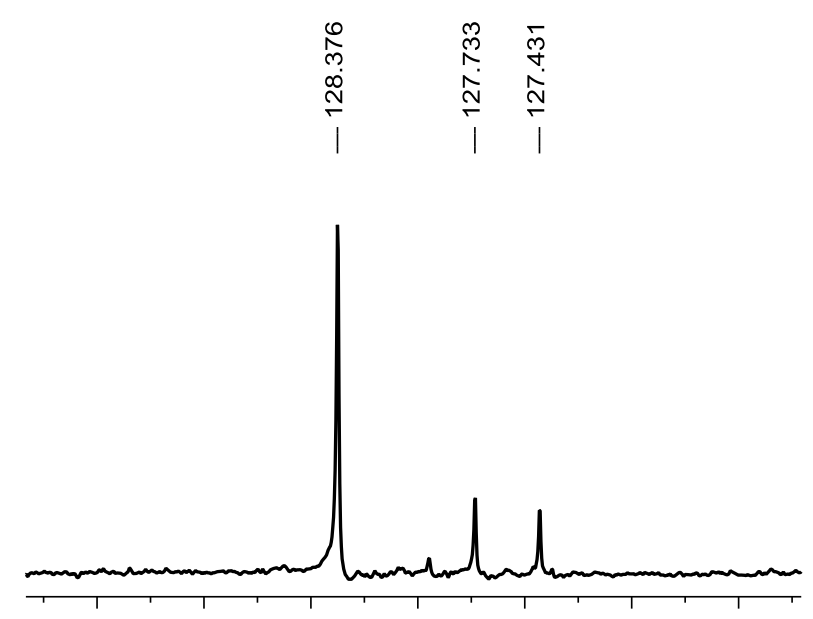

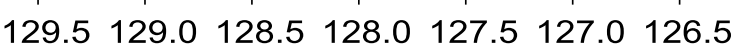

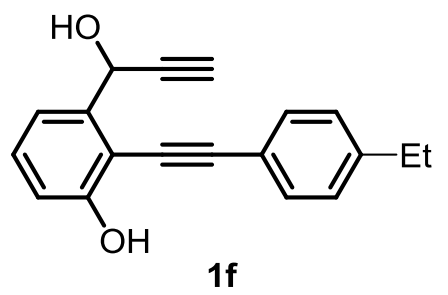

${ }^{13} \mathrm{C}$ NMR $101 \mathrm{MHz}, \mathrm{CDCl}_{3}$

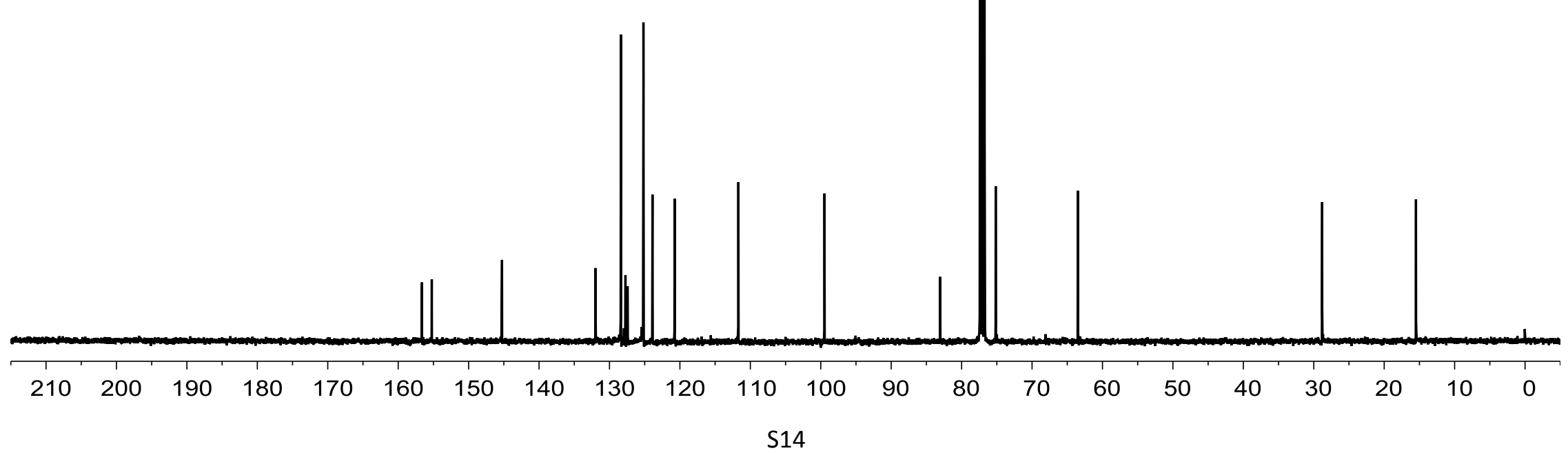




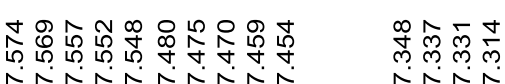
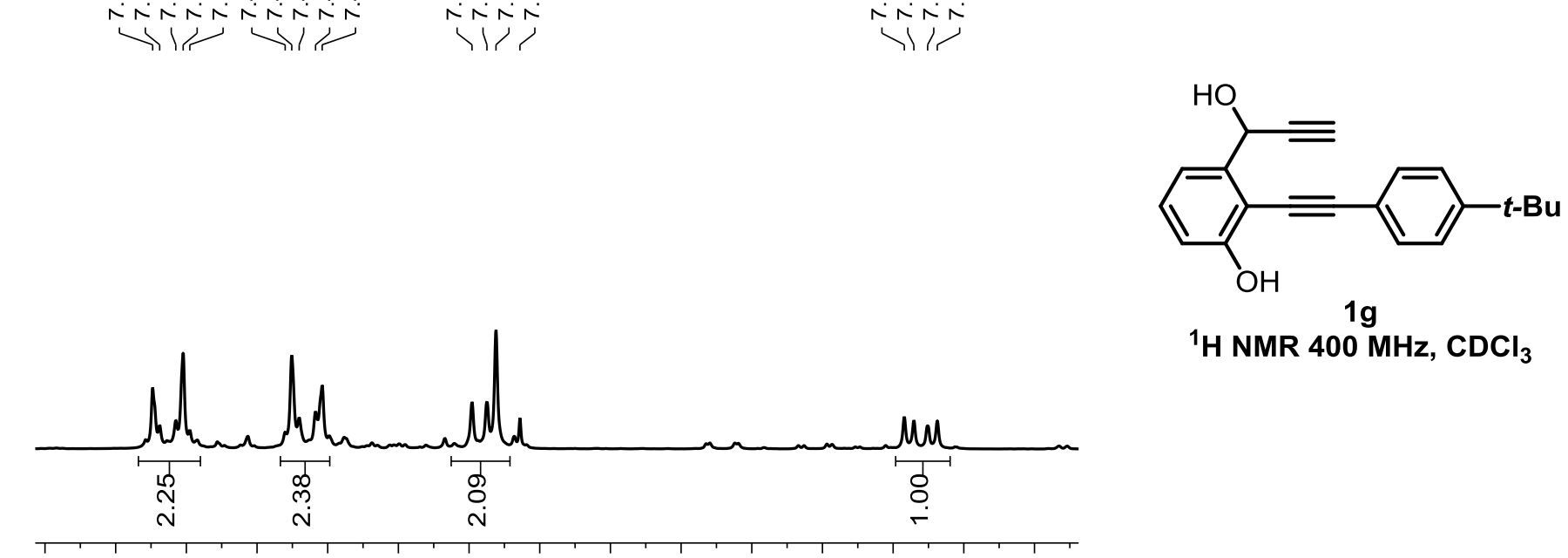

${ }^{1} \mathrm{H}$ NMR $400 \mathrm{MHz}^{1 \mathrm{~g}} \mathrm{CDCl}_{3}$

7.65 7.60 7.55 7.50 7.45 7.40 7.35 7.30 7.25 7.20 7.15 7.10 7.05 7.00 6.95

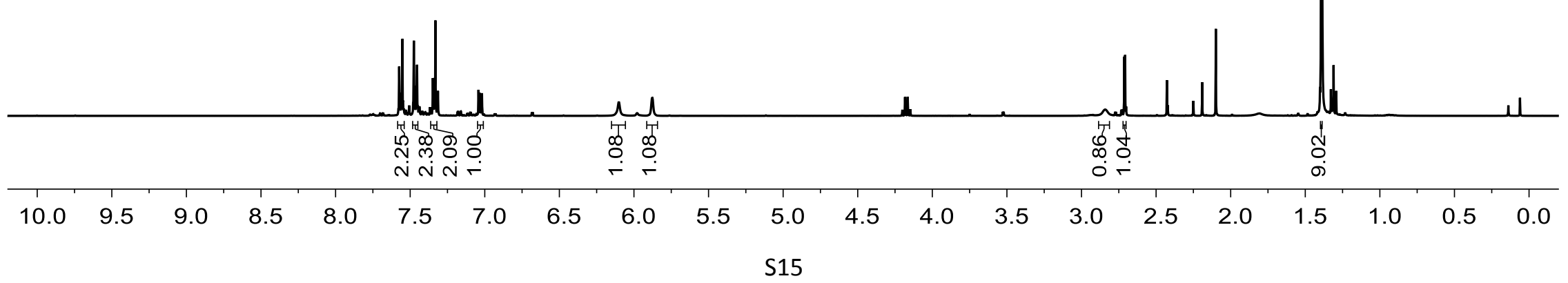




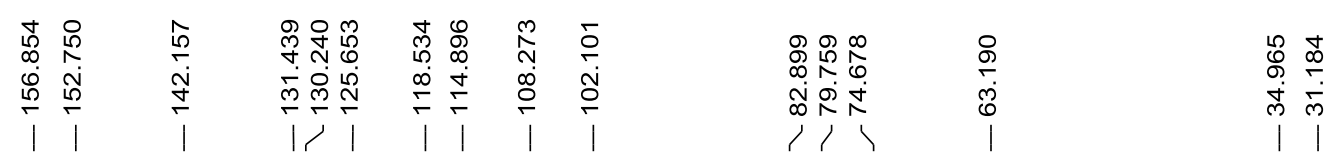
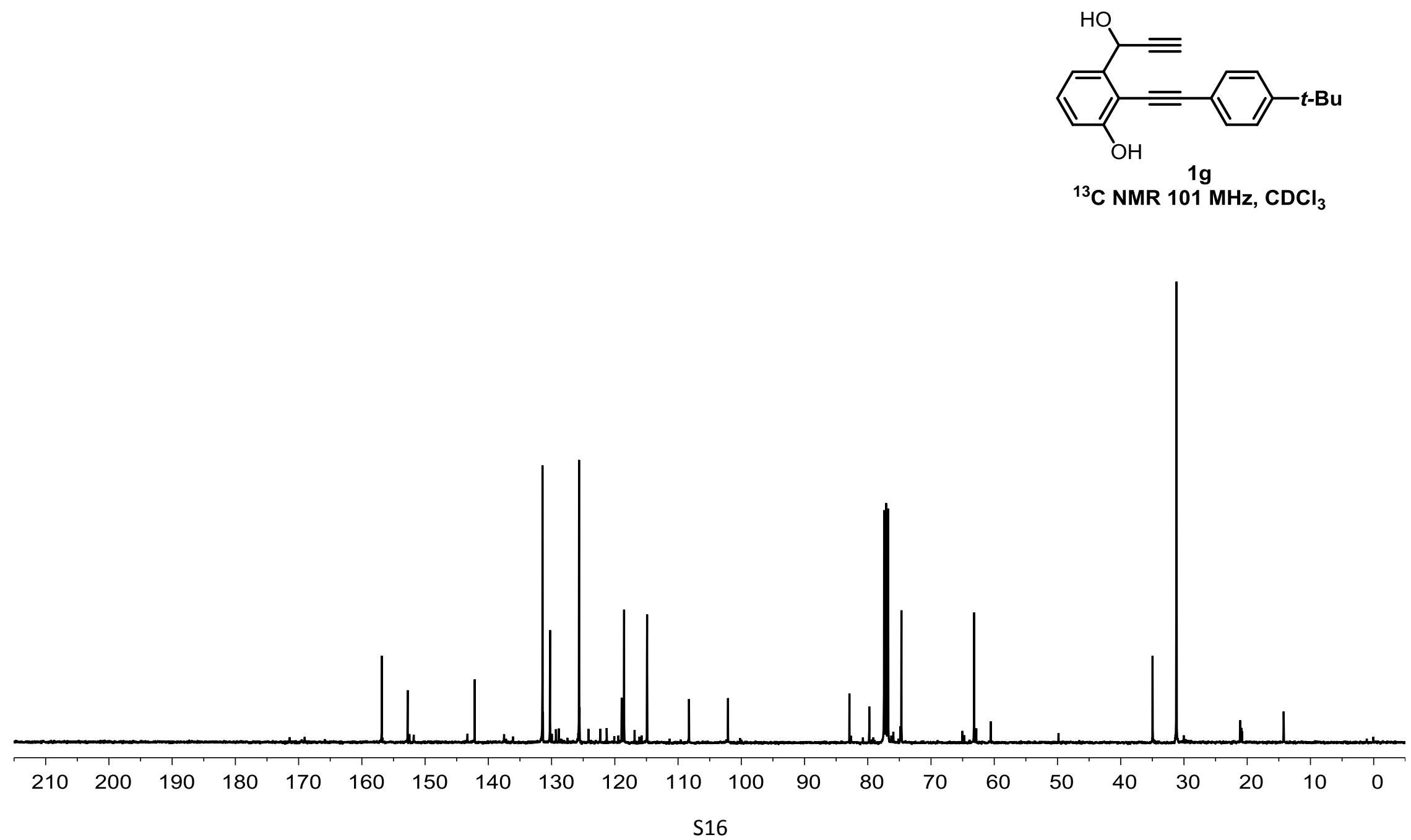


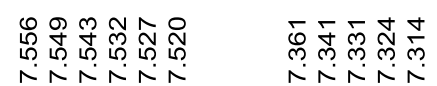

우ㅇㅠㅠ.

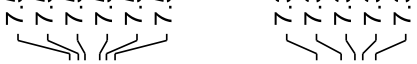

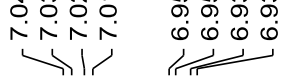
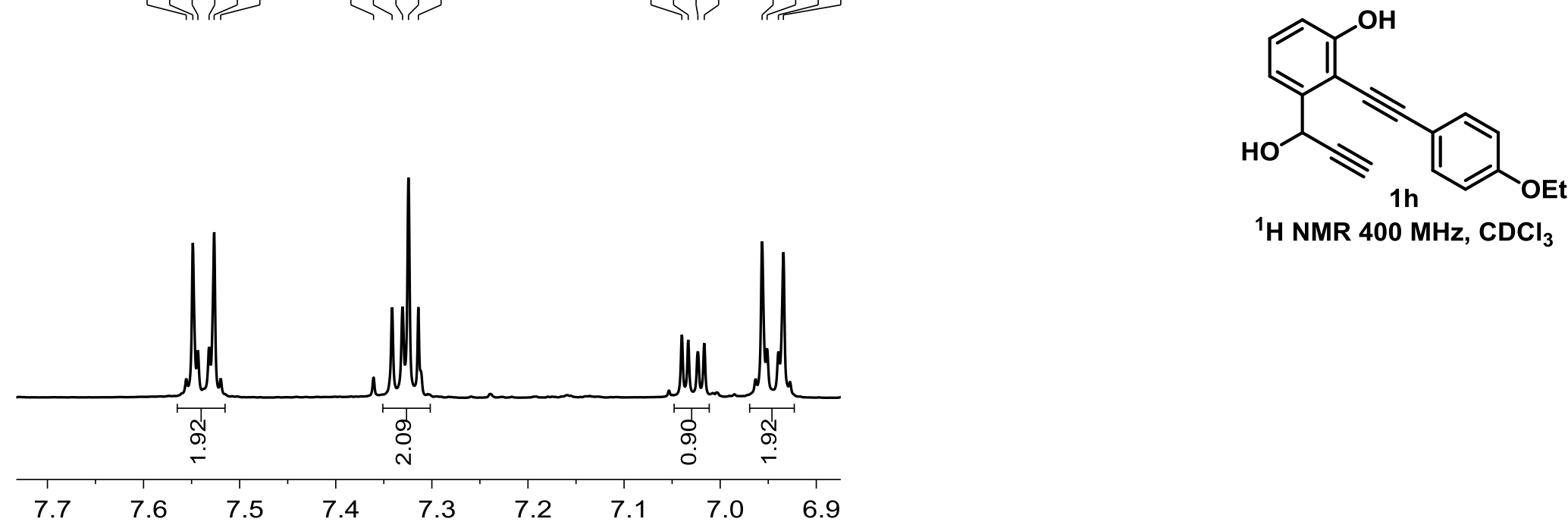

${ }^{1} \mathrm{H}$ NMR $400 \mathrm{MHz}, \mathrm{CDCl}_{3}$

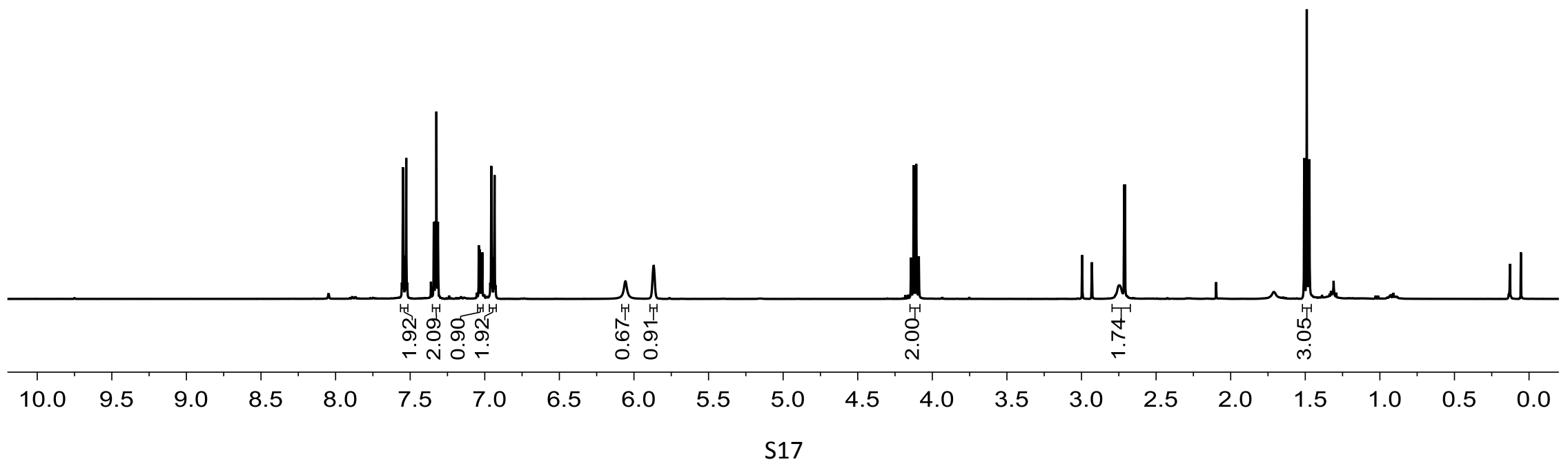




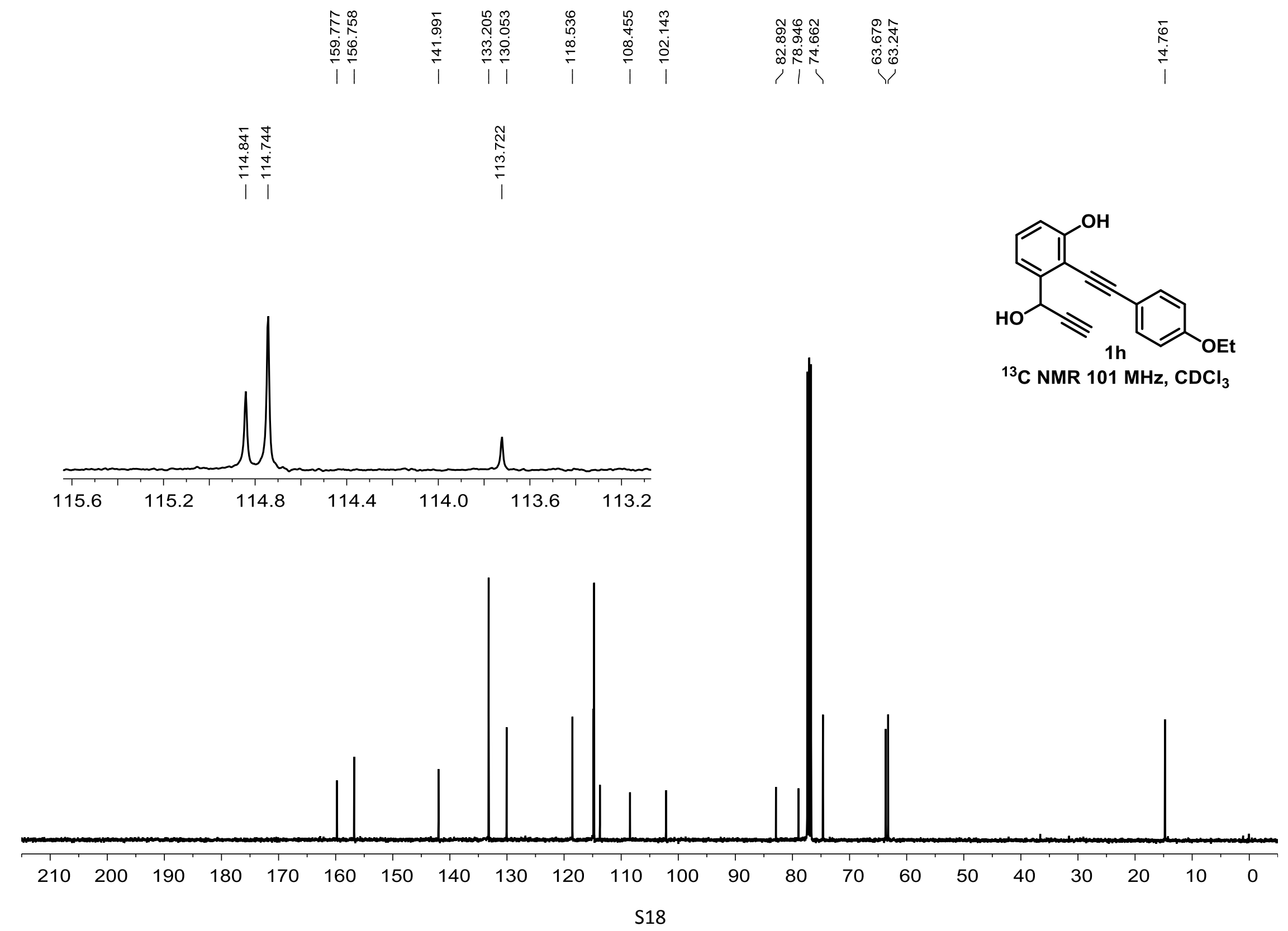




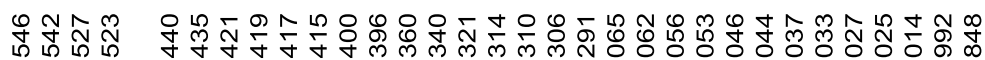

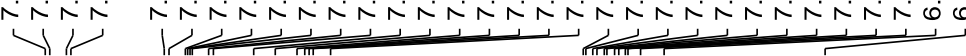
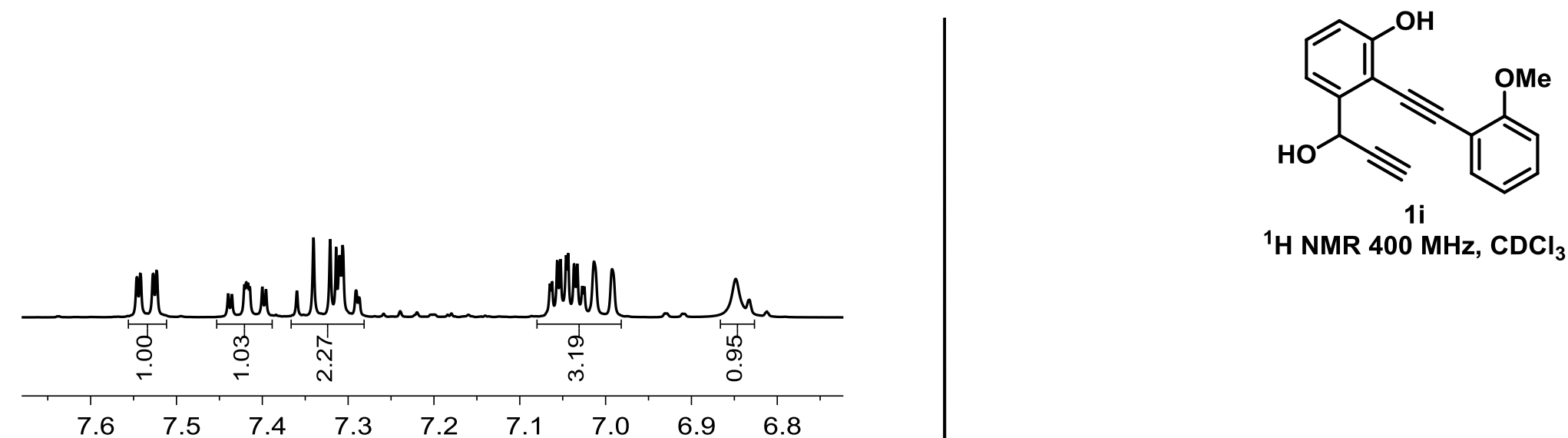

${ }^{1} \mathrm{H}$ NMR $400 \mathrm{MHz}, \mathrm{CDCl}_{3}$

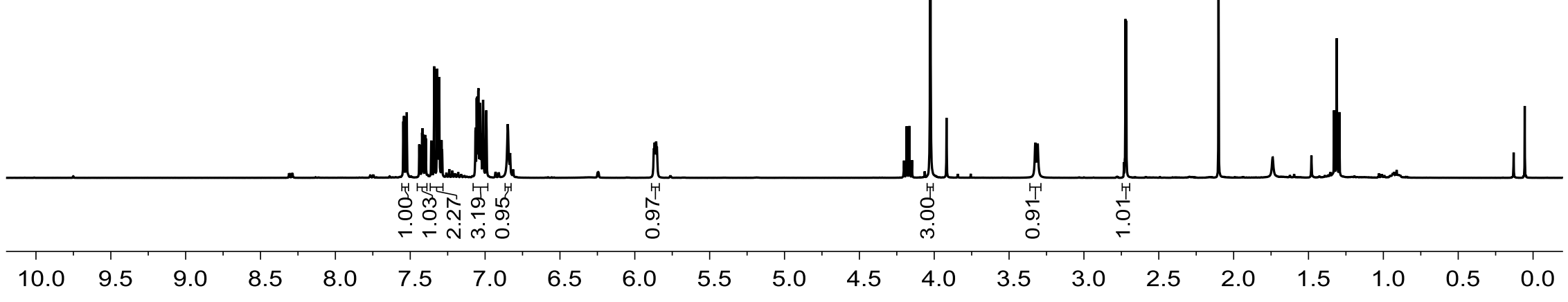




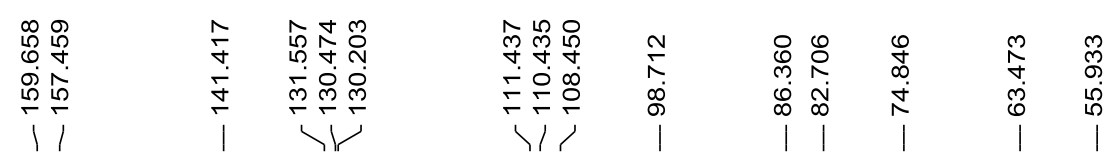

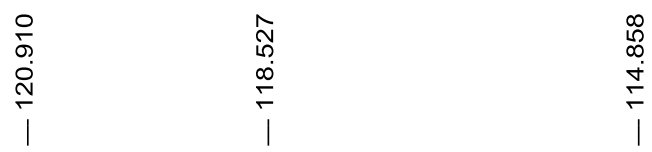
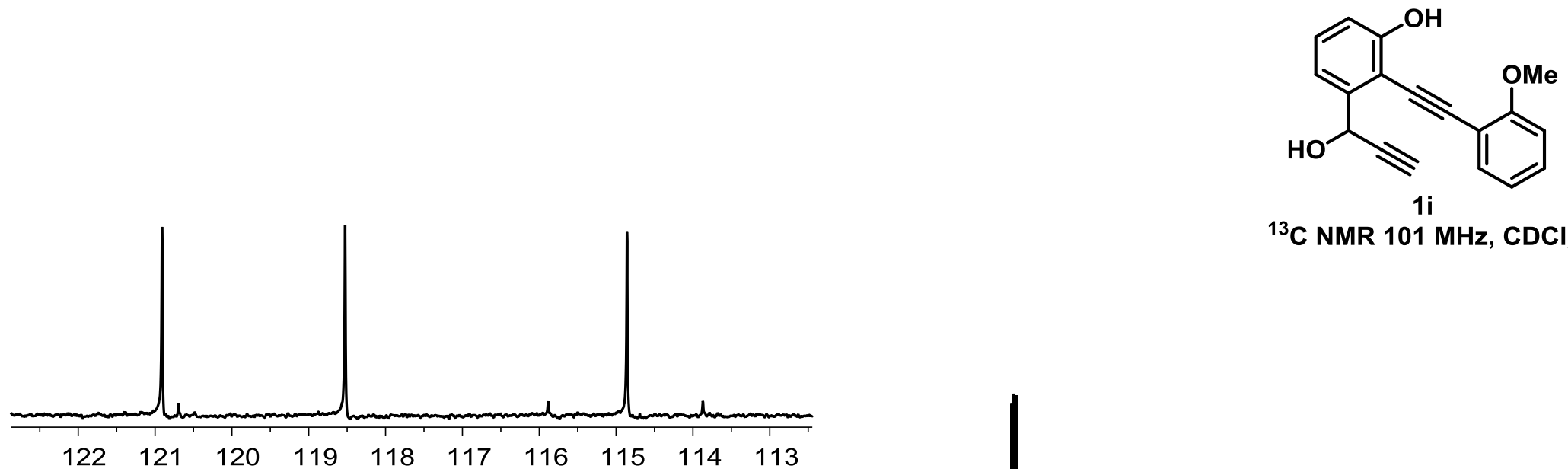

${ }^{13} \mathrm{C}$ NMR $101 \mathrm{MHz}, \mathrm{CDCl}_{3}$

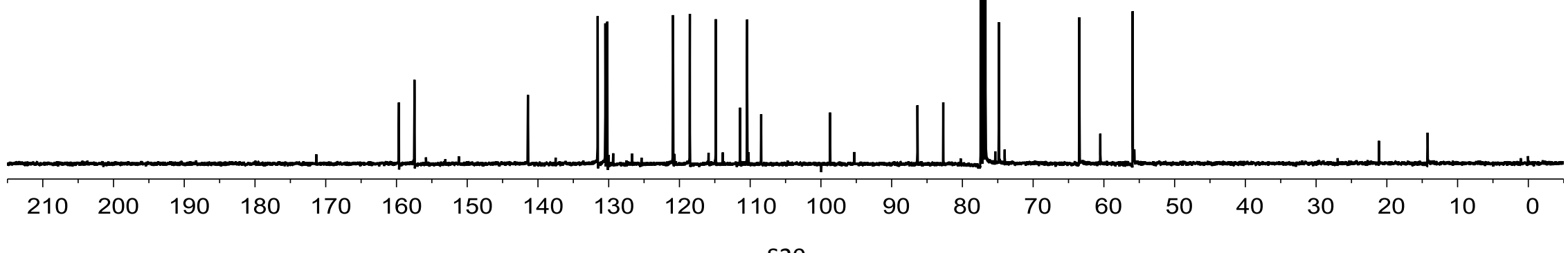




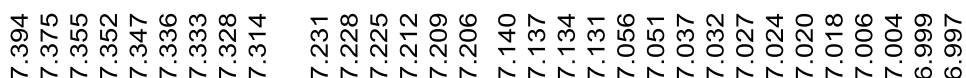

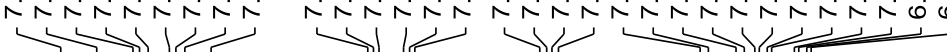

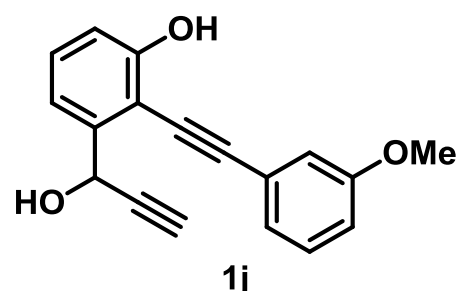

${ }^{1} \mathrm{H}$ NMR $400 \mathrm{MHz}, \mathrm{CDCl}_{3}$

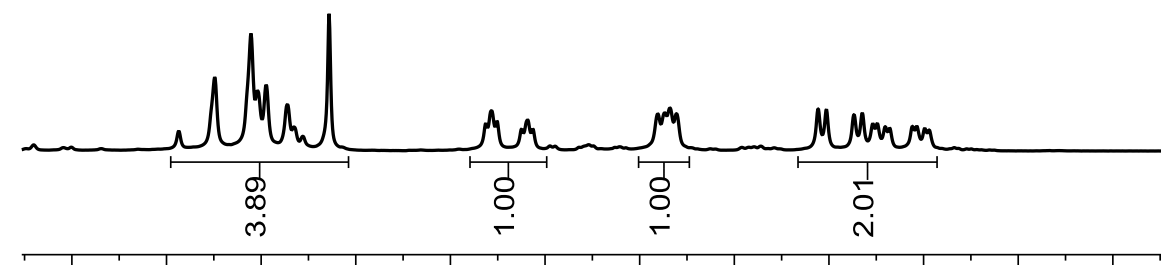

$\begin{array}{llllllllllll}7.45 & 7.40 & 7.35 & 7.30 & 7.25 & 7.20 & 7.15 & 7.10 & 7.05 & 7.00 & 6.95 & 6.90\end{array}$

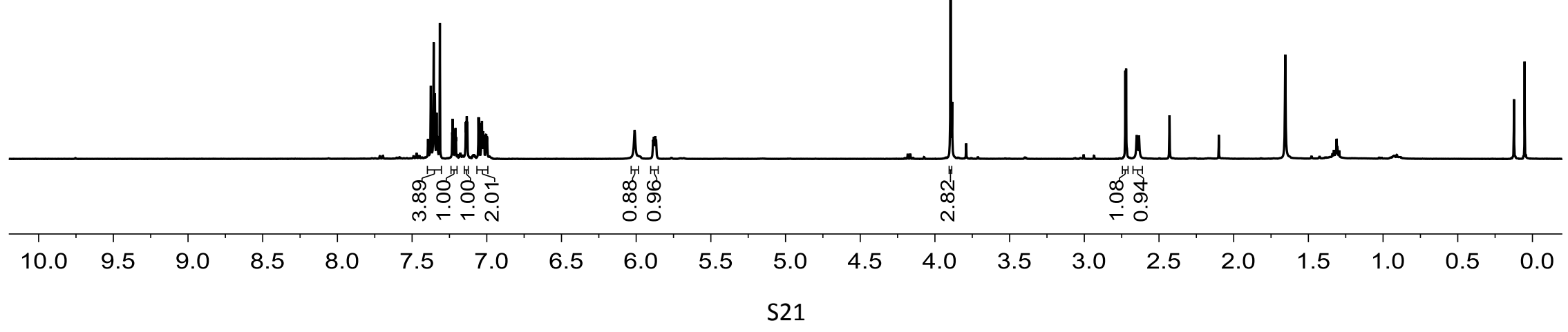




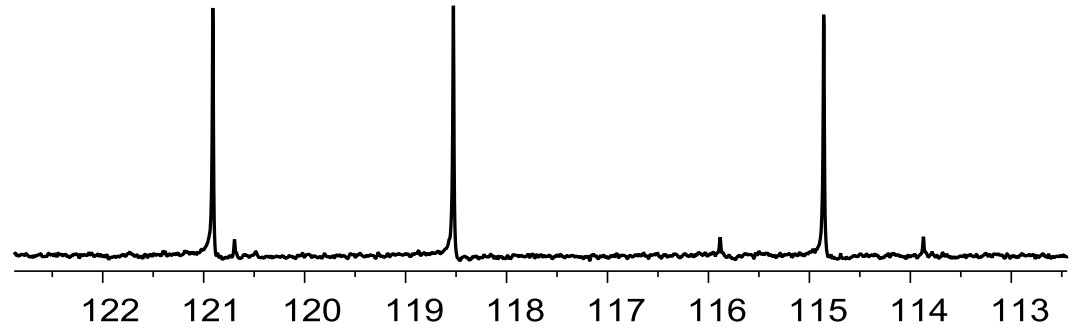

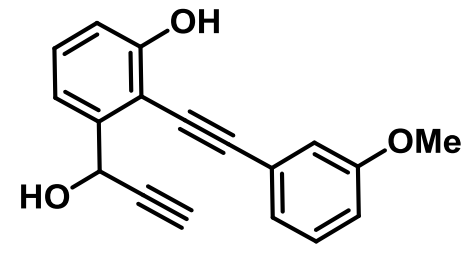

$1 \mathrm{j}$

${ }^{13} \mathrm{C}$ NMR $101 \mathrm{MHz}, \mathrm{CDCl}_{3}$

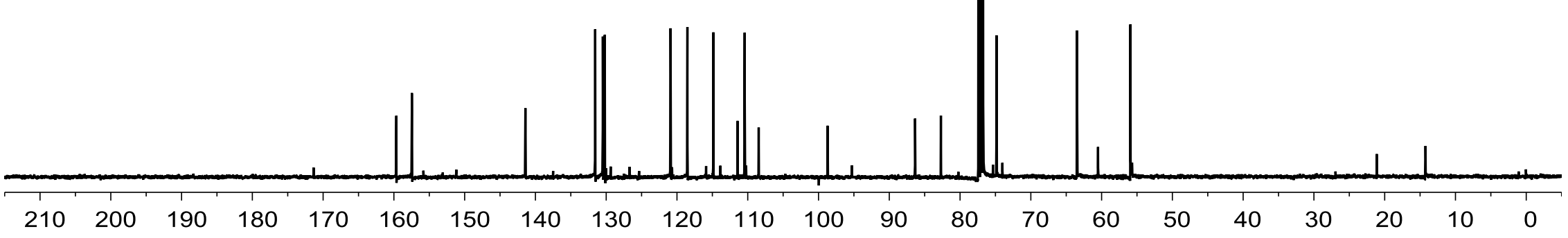




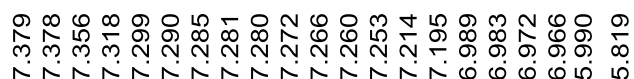

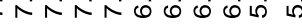

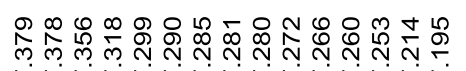

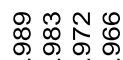
(

○ं

प1 1

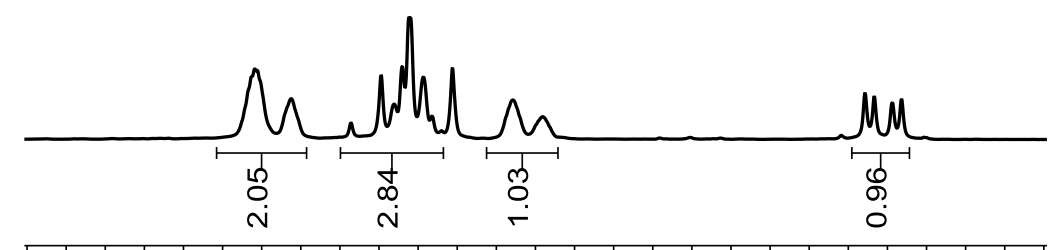

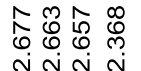

7.50 7.45 7.40 7.35 7.30 7.25 7.20 7.15 7.10 7.05 7.00 6.95 6.90

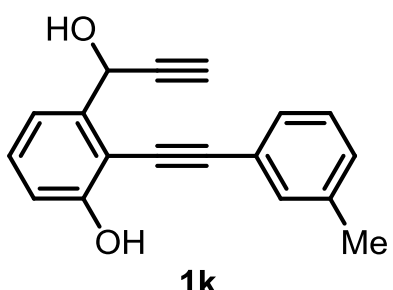

${ }^{1} \mathrm{H}$ NMR $400 \mathrm{MHz}^{\mathrm{CDCl}_{3}}$

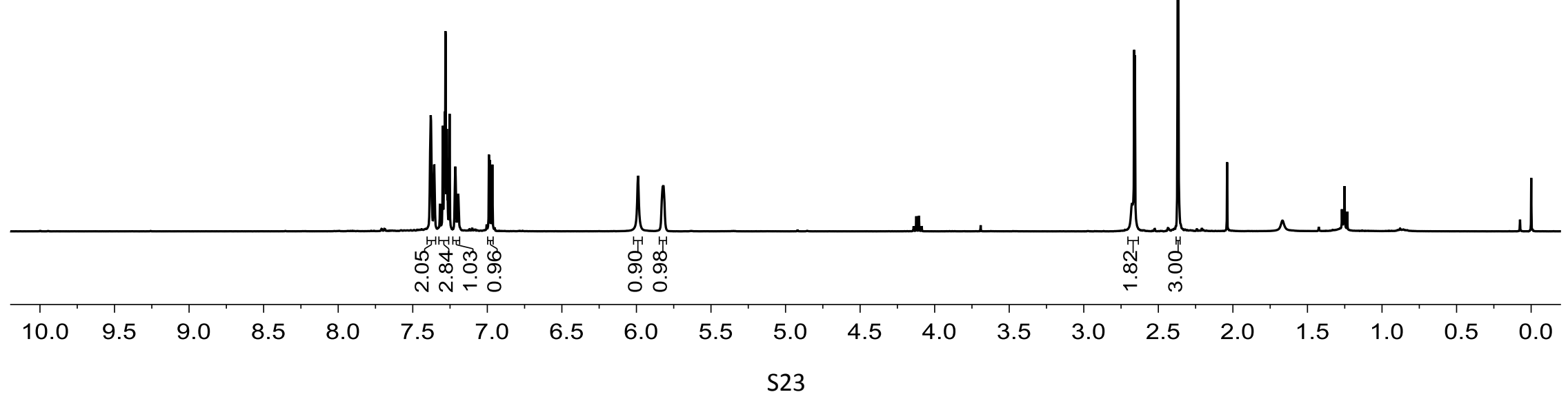




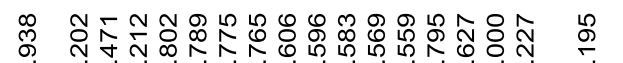

传
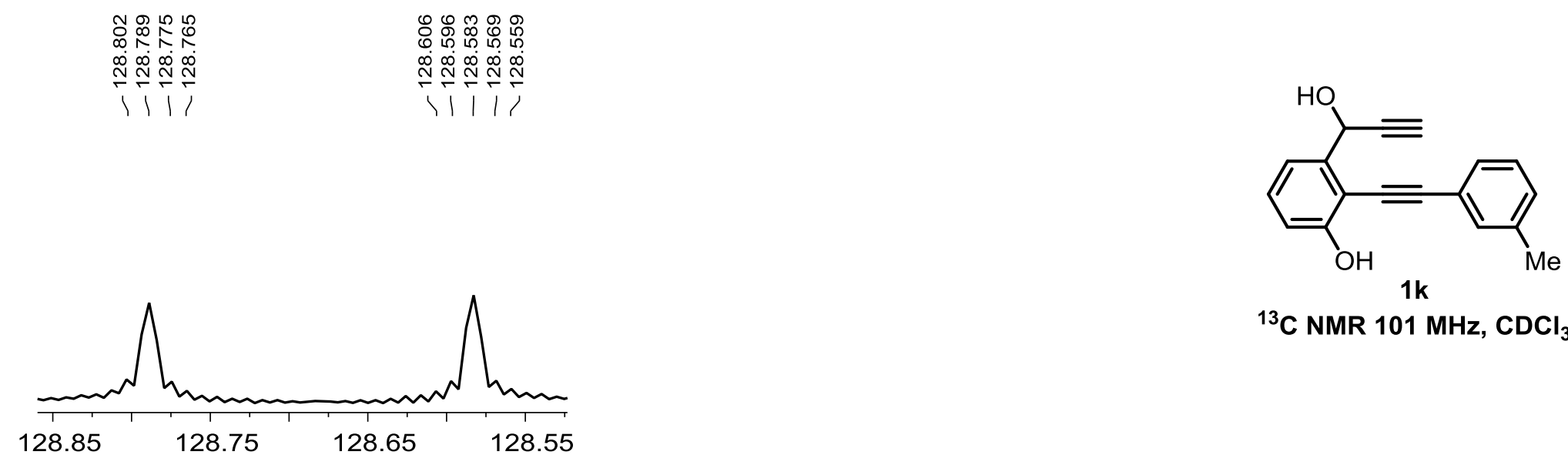

${ }^{13} \mathrm{C}$ NMR $101 \mathrm{MHz}, \mathrm{CDCl}_{3}$

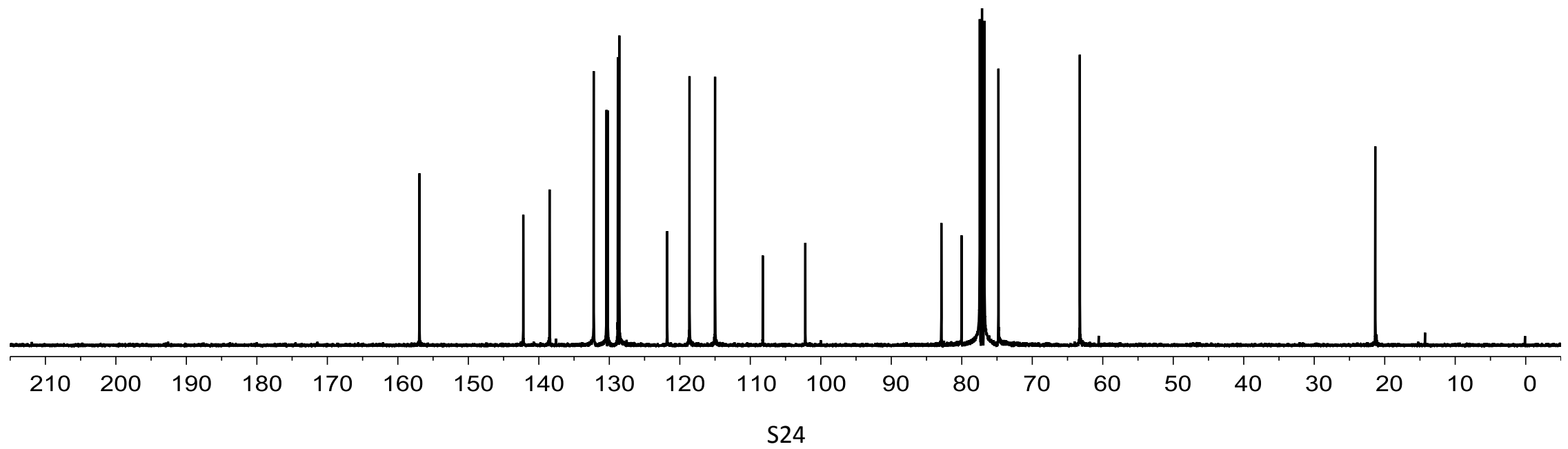




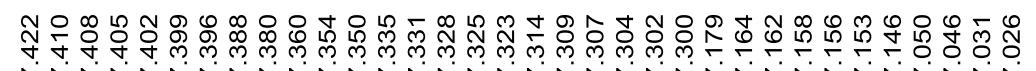

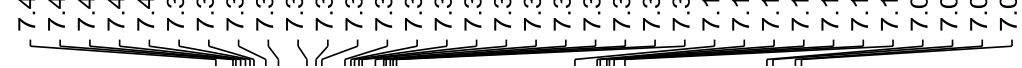

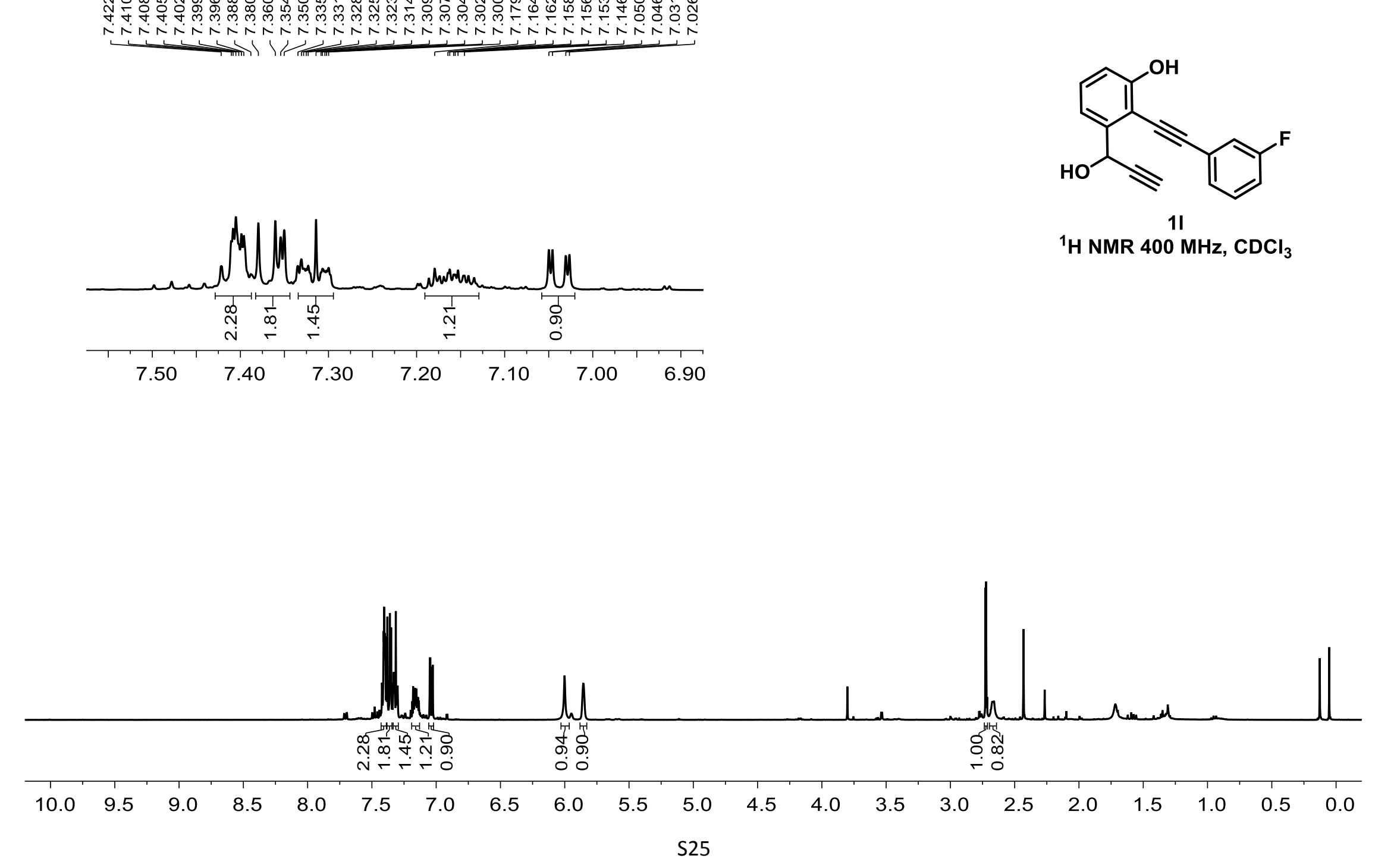




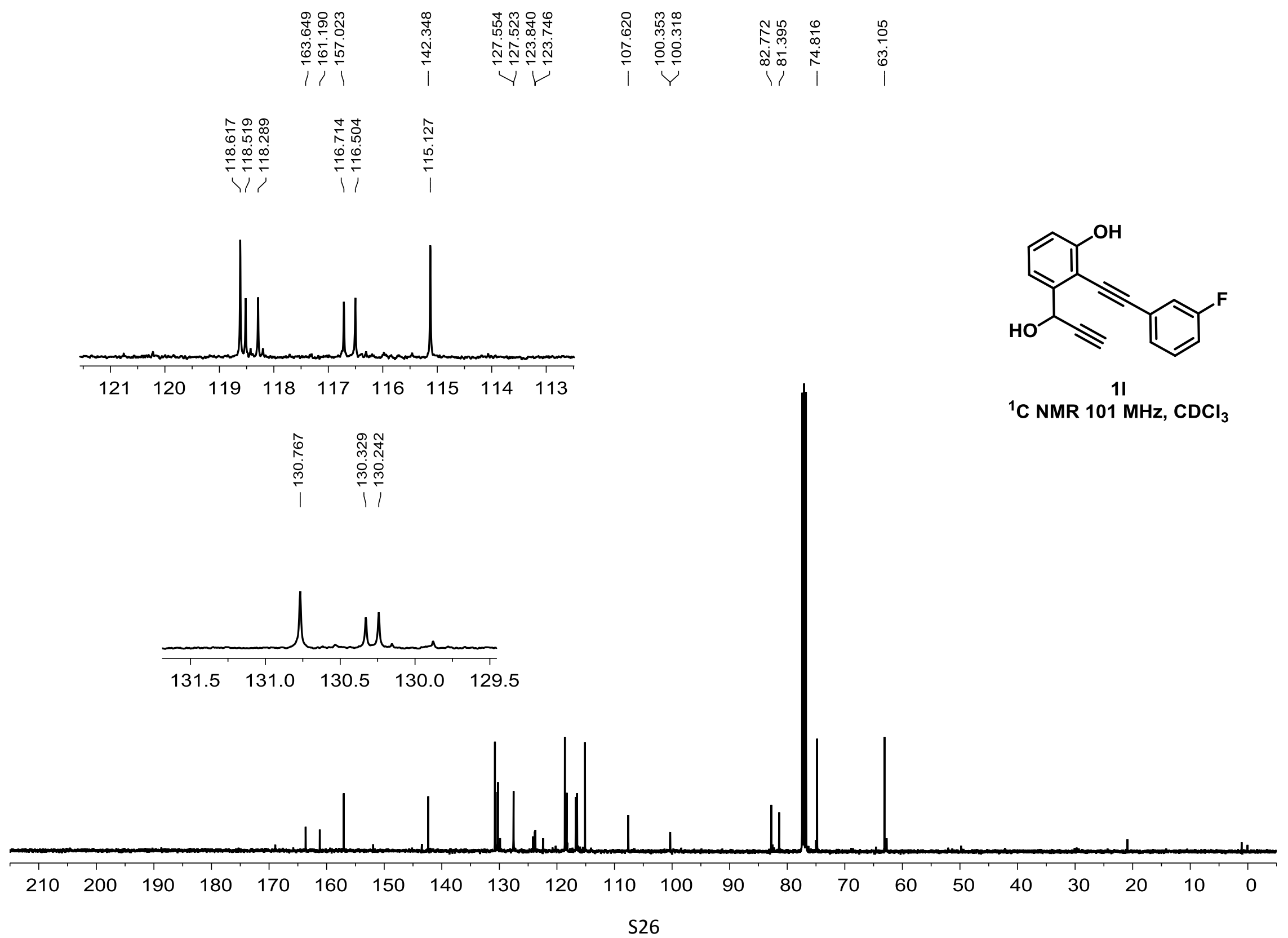




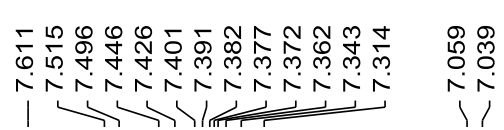

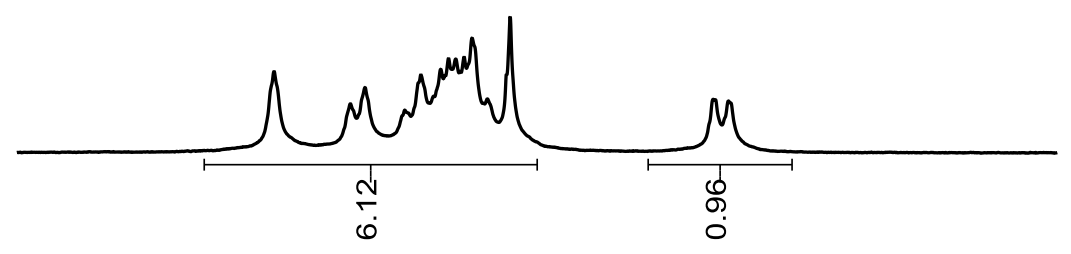

$\begin{array}{llllllllllllll}7.9 & 7.8 & 7.7 & 7.6 & 7.5 & 7.4 & 7.3 & 7.2 & 7.1 & 7.0 & 6.9 & 6.8 & 6.7\end{array}$

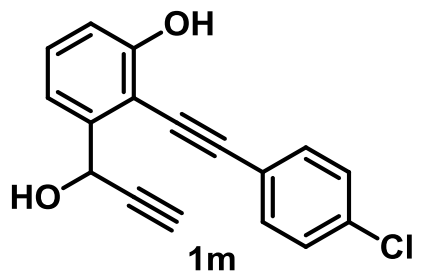

${ }^{1} \mathrm{H}$ NMR $400 \mathrm{MHz}^{\mathrm{CDCl}}{ }_{3}$

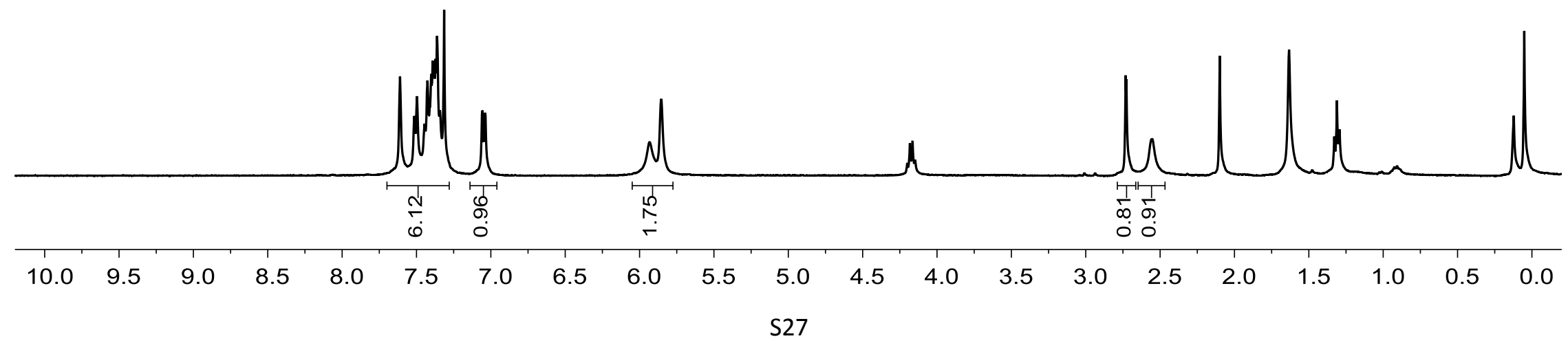




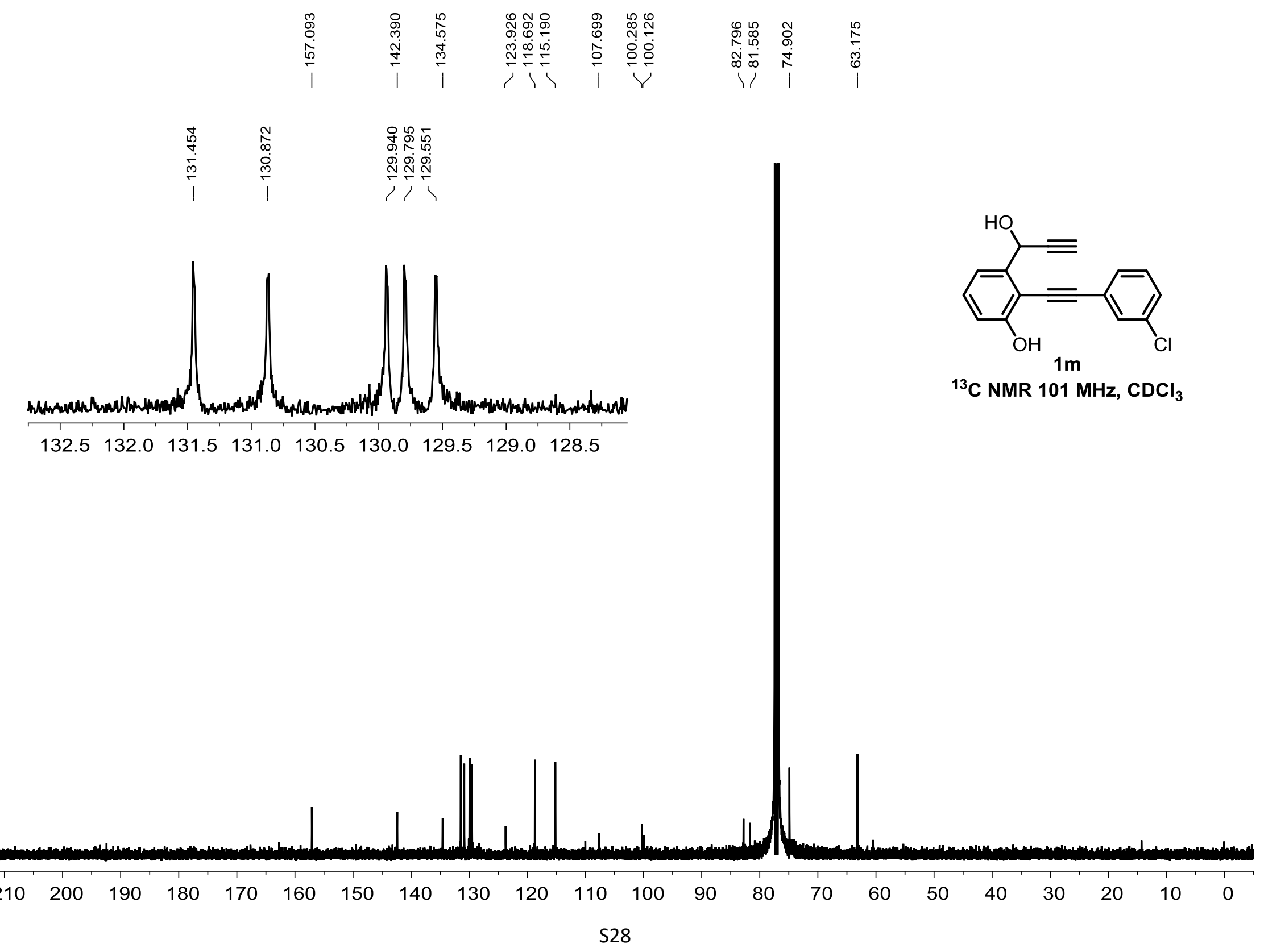




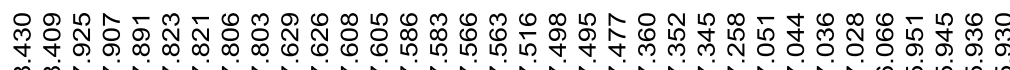

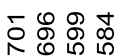

ヘ
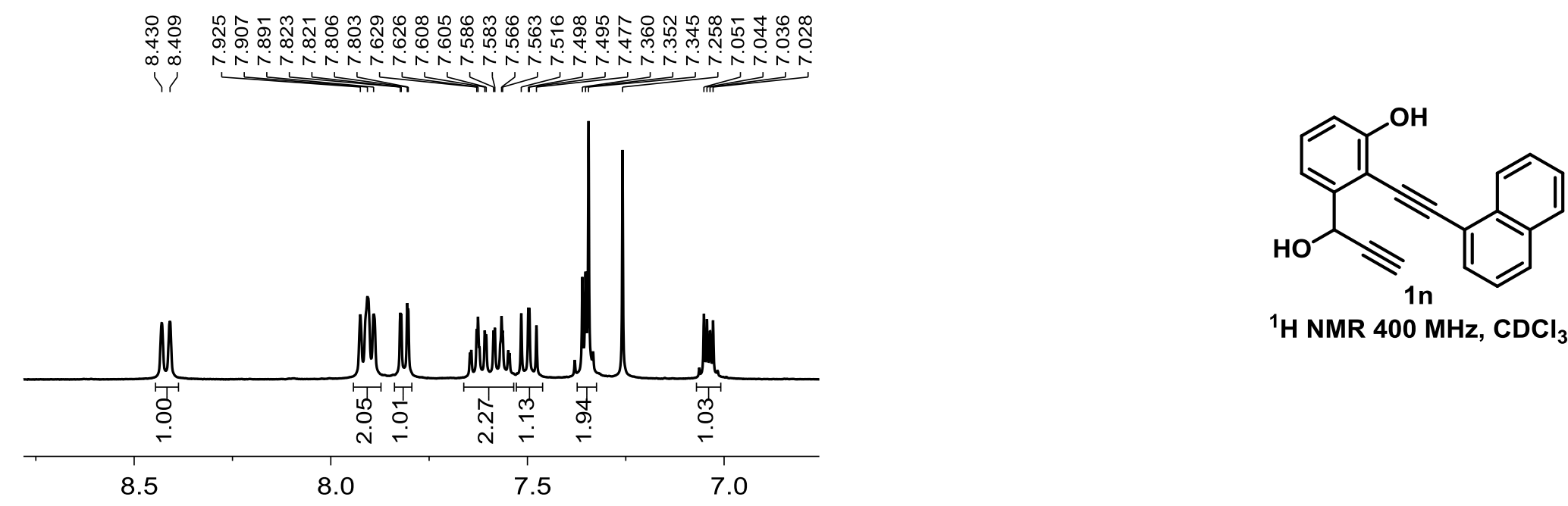

${ }^{1} \mathrm{H}$ NMR $400 \mathrm{MHz}, \mathrm{CDCl}_{3}$

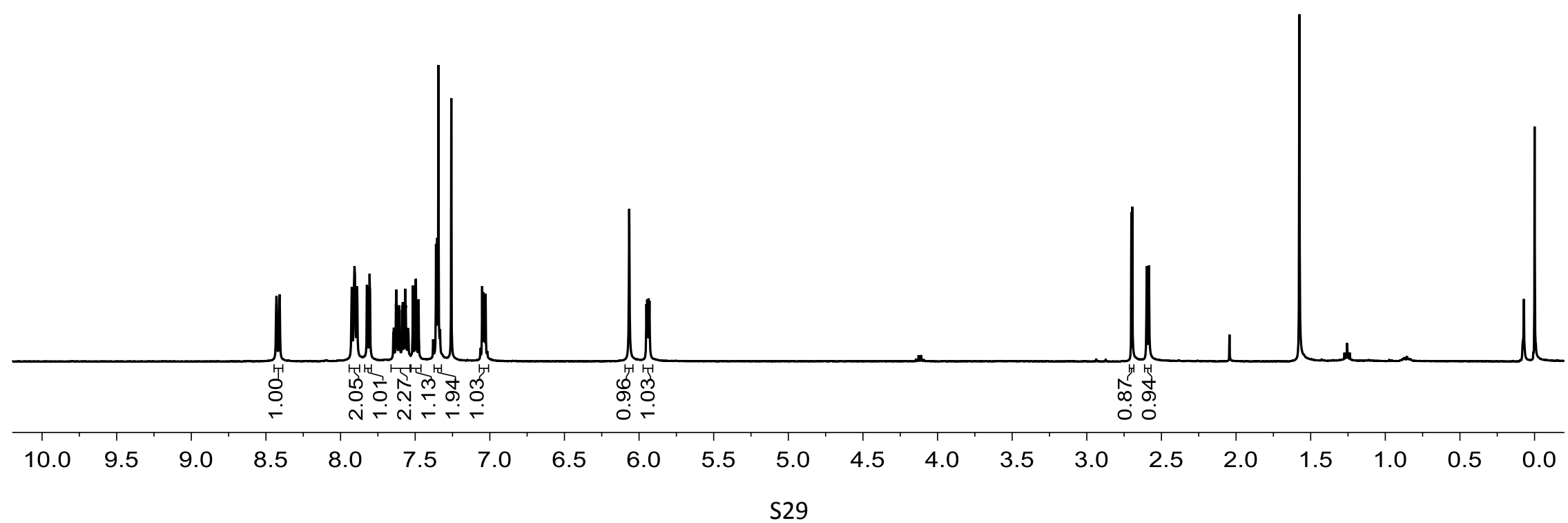




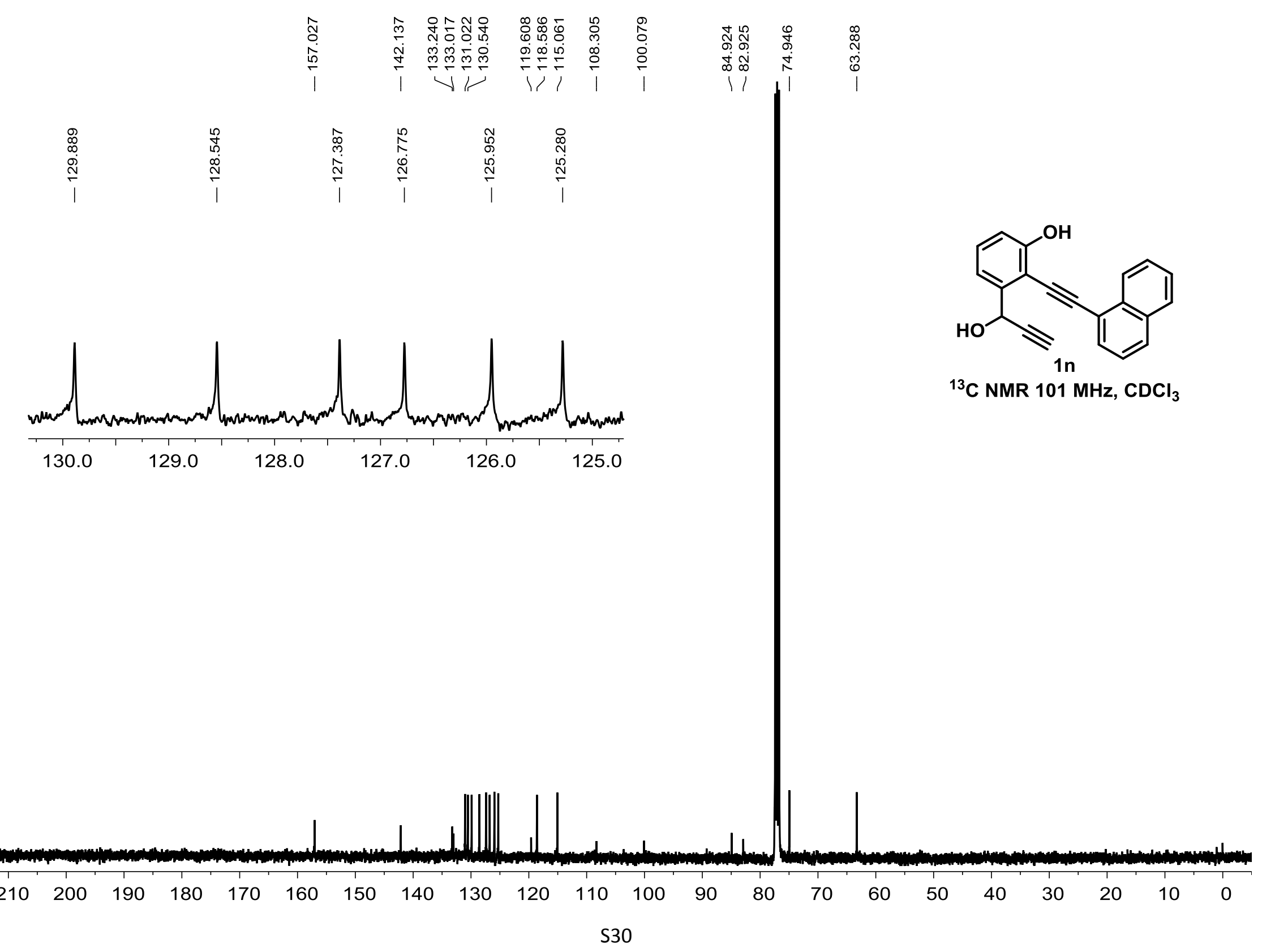




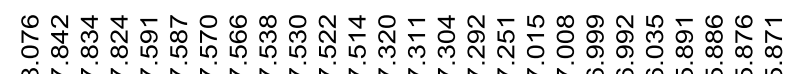

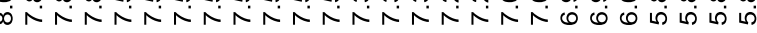

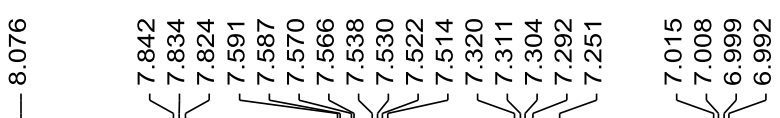
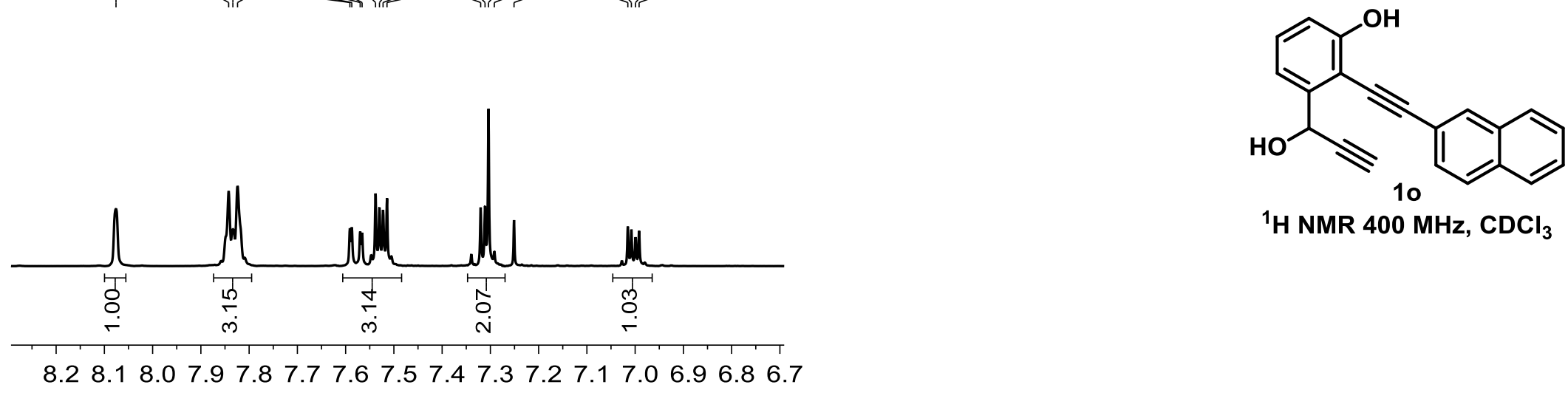

${ }^{1} \mathrm{H}$ NMR $400 \mathrm{MHz}, \mathrm{CDCl}_{3}$

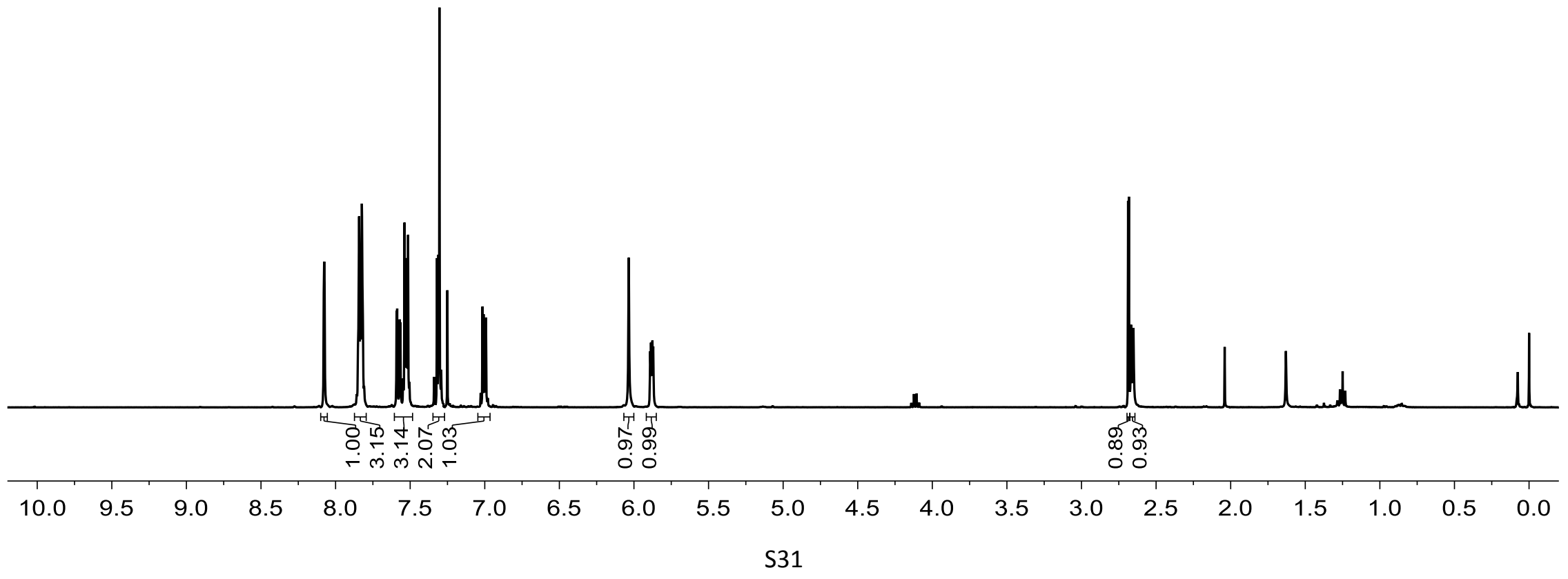




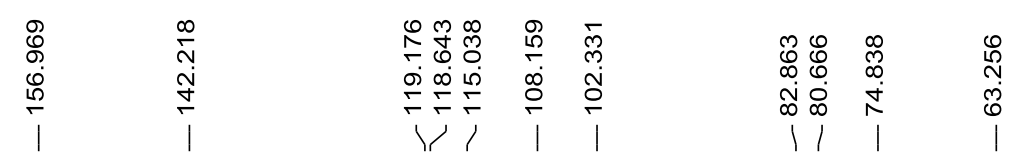

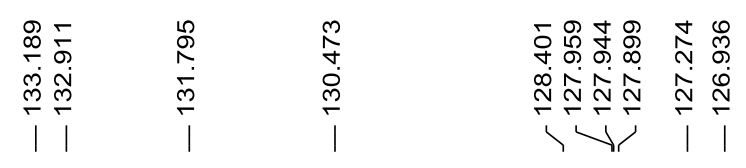
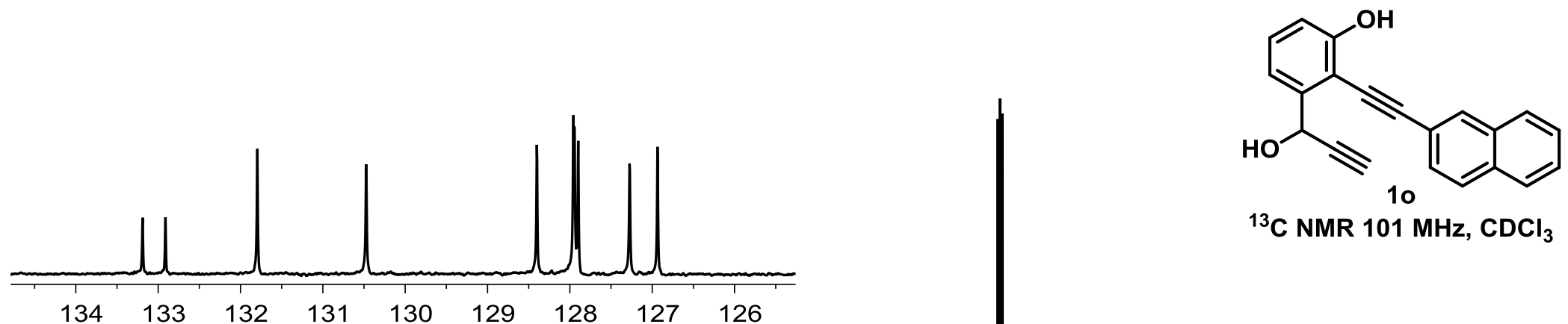

${ }^{13} \mathrm{C} \mathrm{NMR} 101 \mathrm{MHz}, \mathrm{CDCl}_{3}$

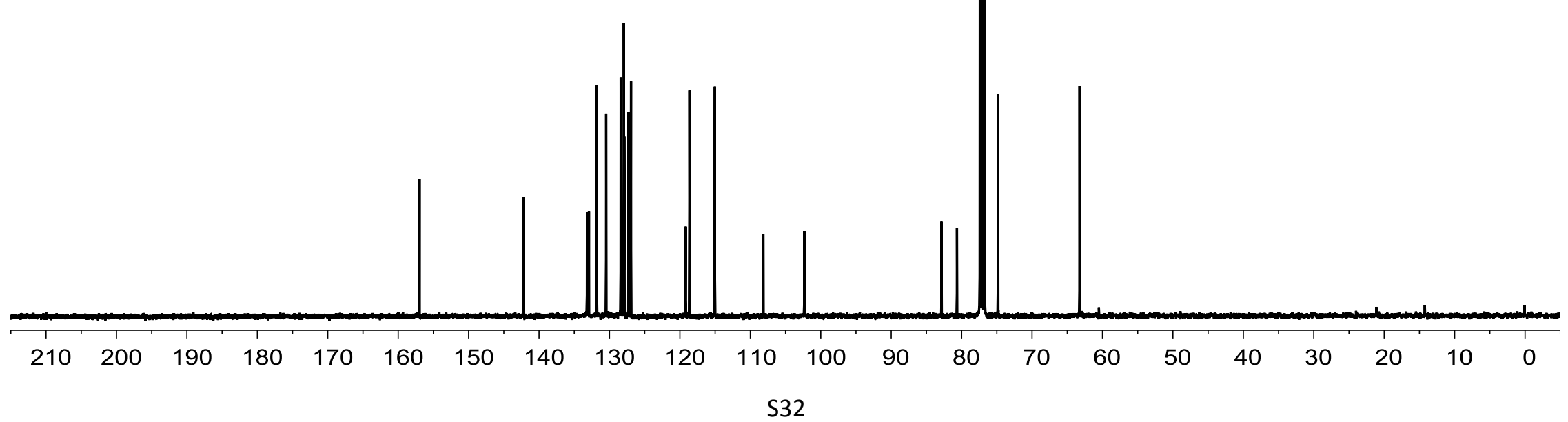




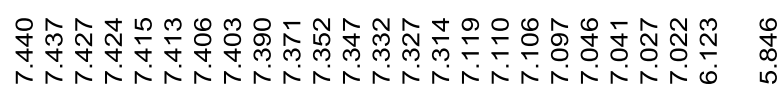

$\underset{\substack{N \\ N}}{N}$

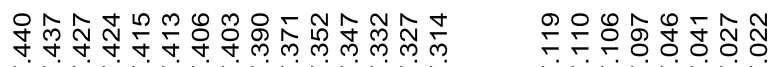

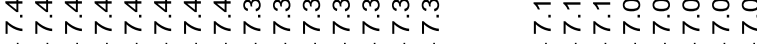

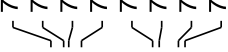
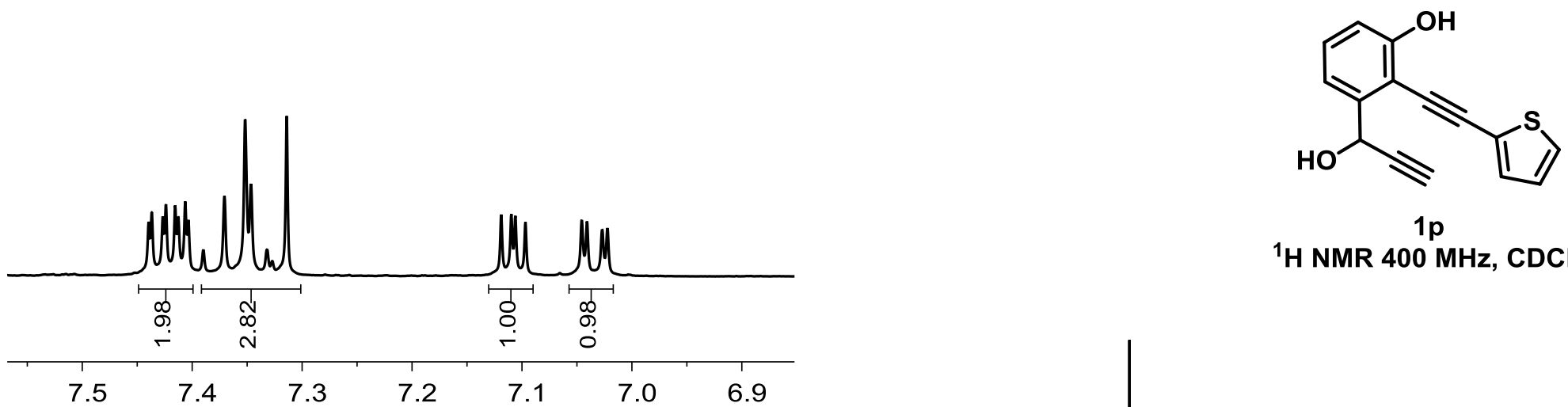

$1 \mathrm{p}$

${ }^{1} \mathrm{H}$ NMR $400 \mathrm{MHz}, \mathrm{CDCl}_{3}$

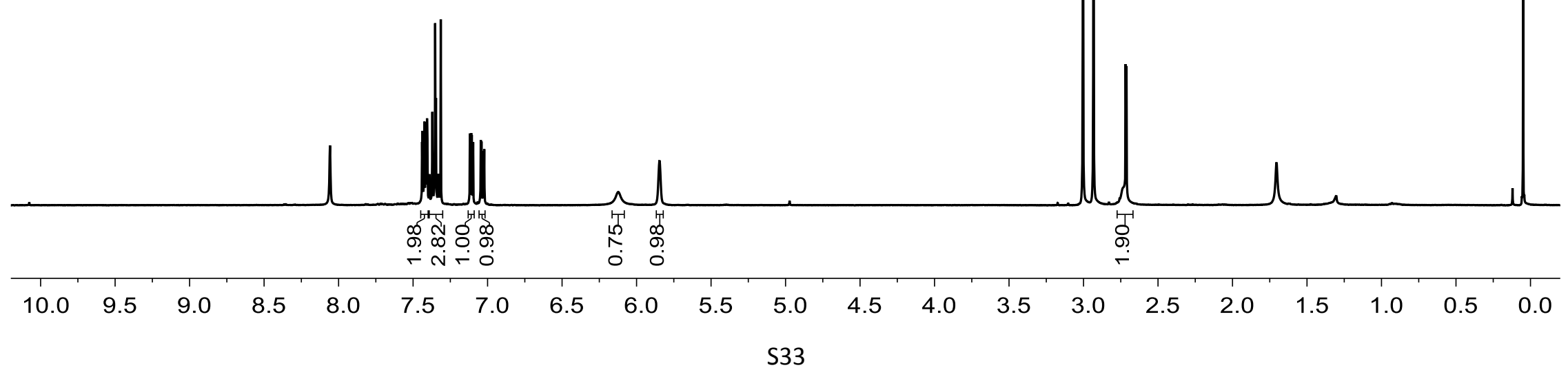




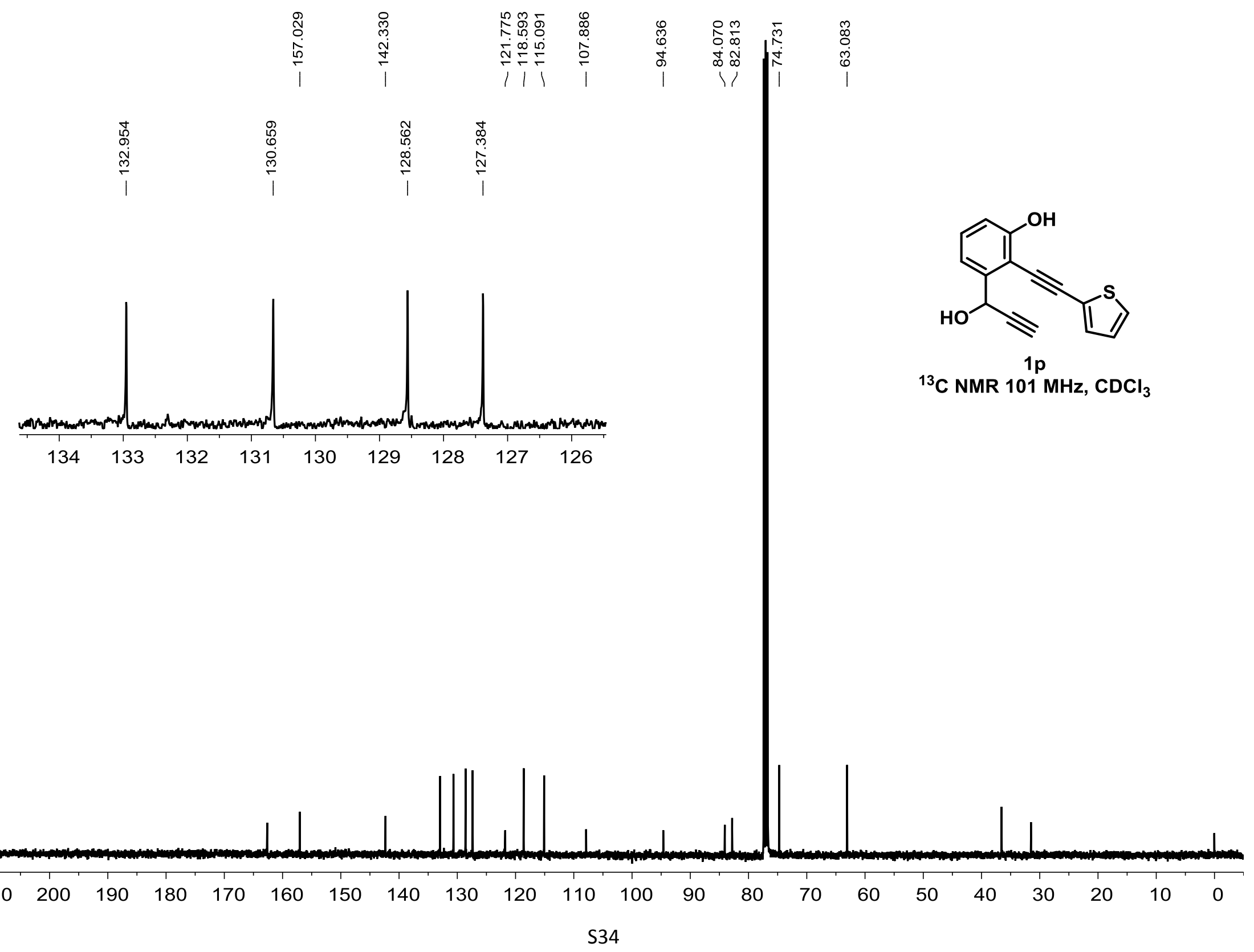




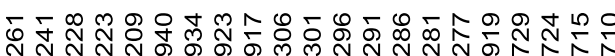

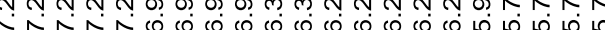

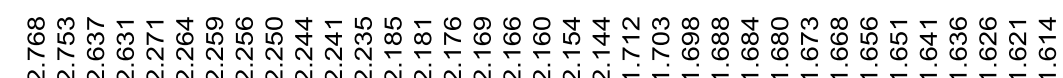
ง
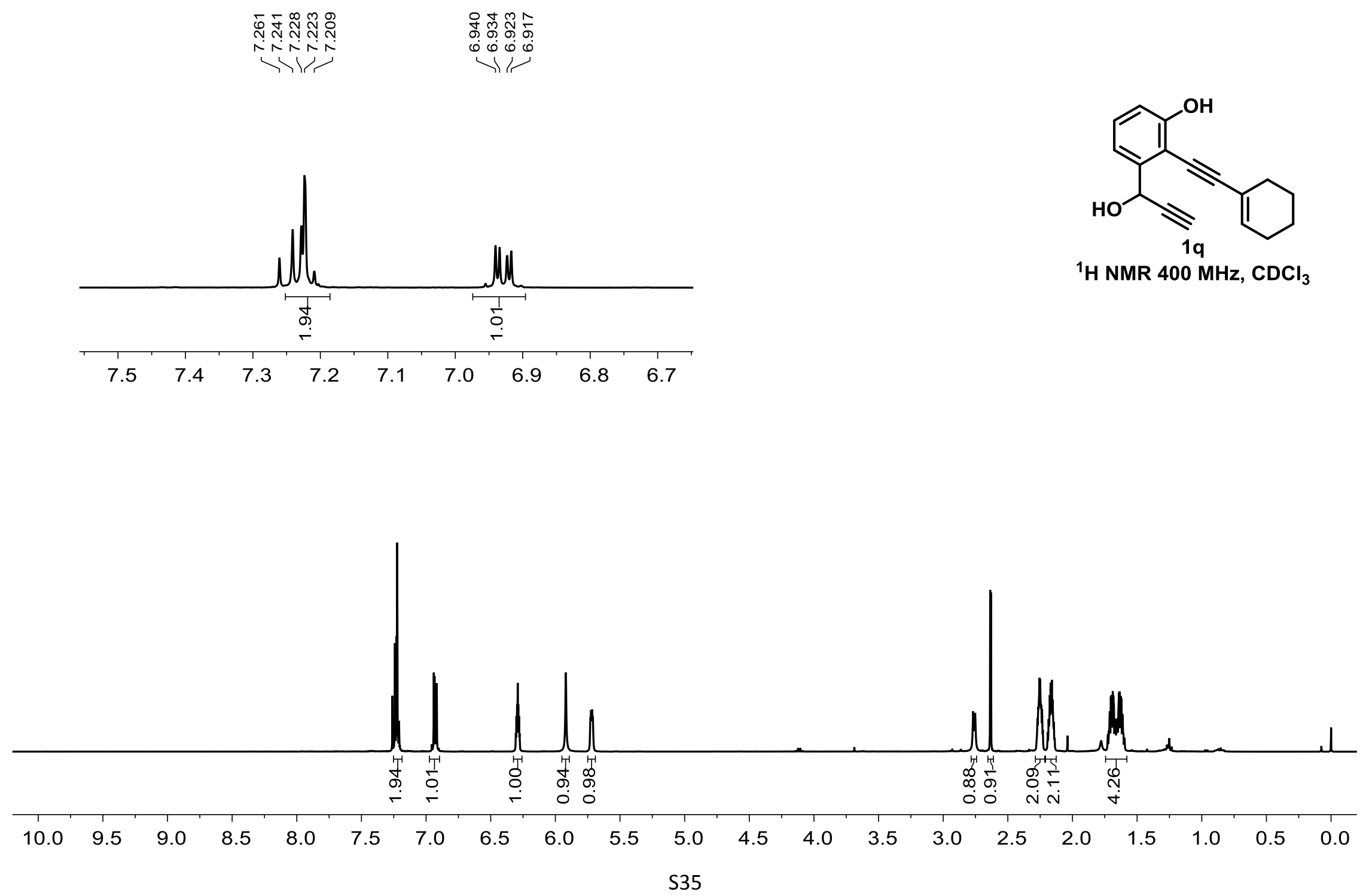


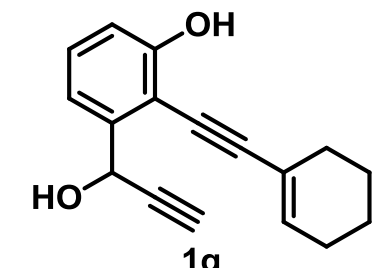

${ }^{13} \mathrm{C}$ NMR $101 \mathrm{MHz}, \mathrm{CDCl}_{3}$

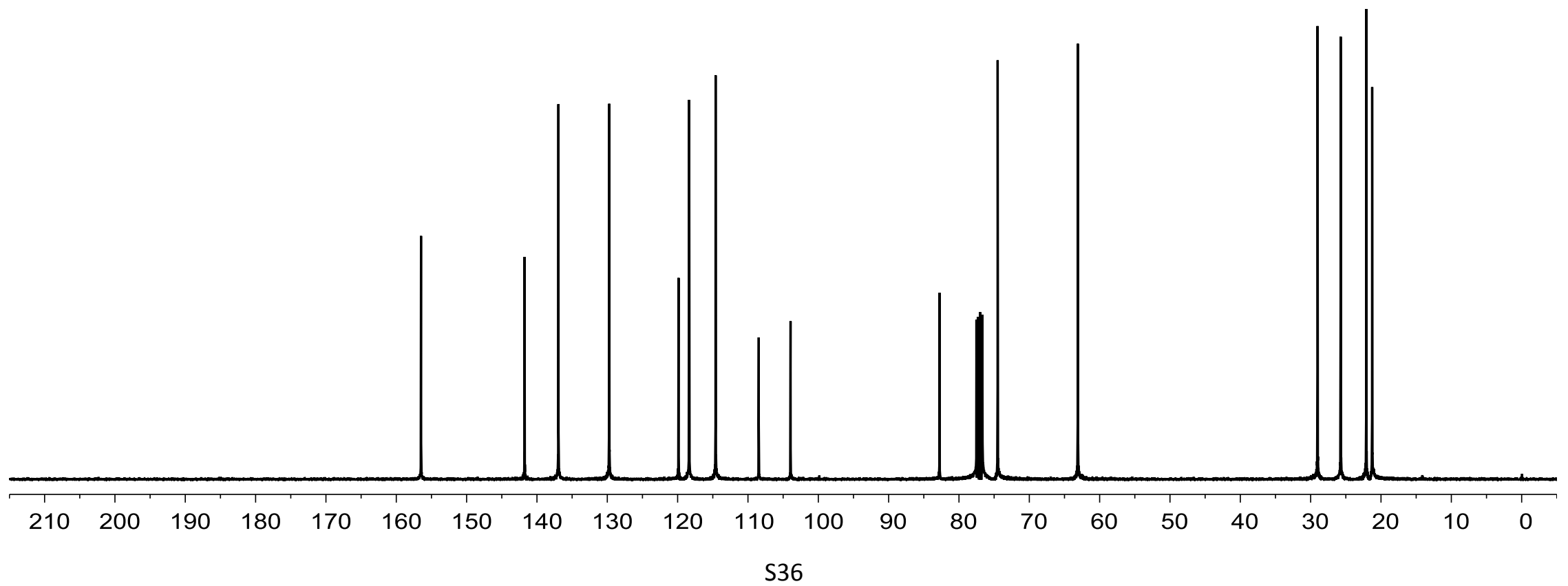




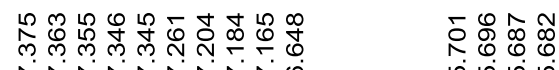

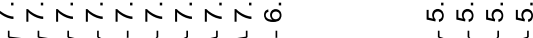

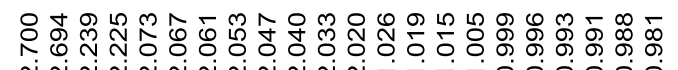

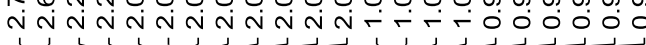

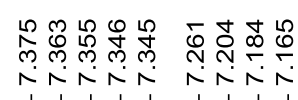

पiए, पर

$\infty$
$\vdots$
0
0
1
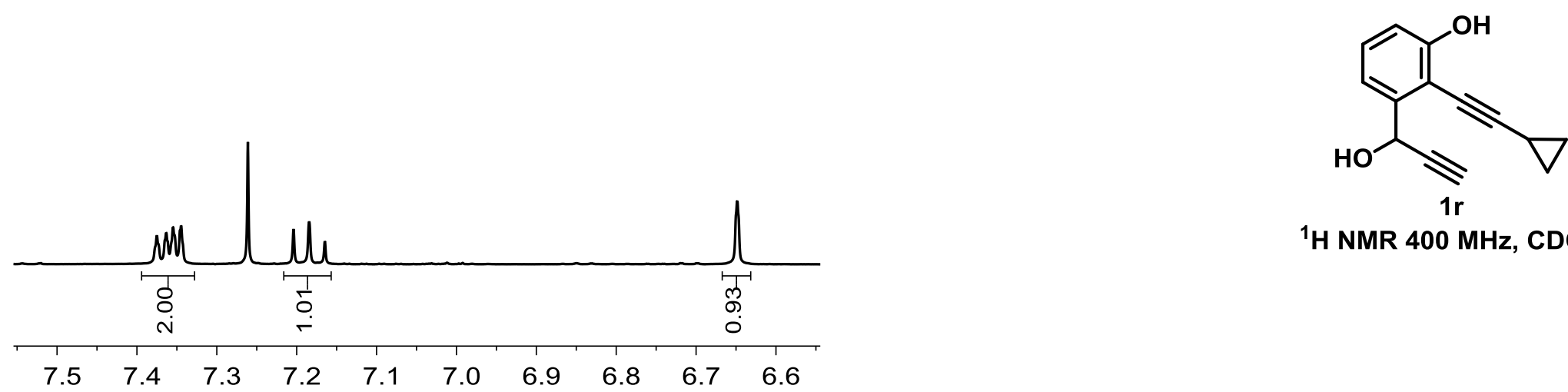

${ }^{1} \mathrm{H}$ NMR $400 \mathrm{MHz}^{\mathrm{CDCl}}{ }_{3}$

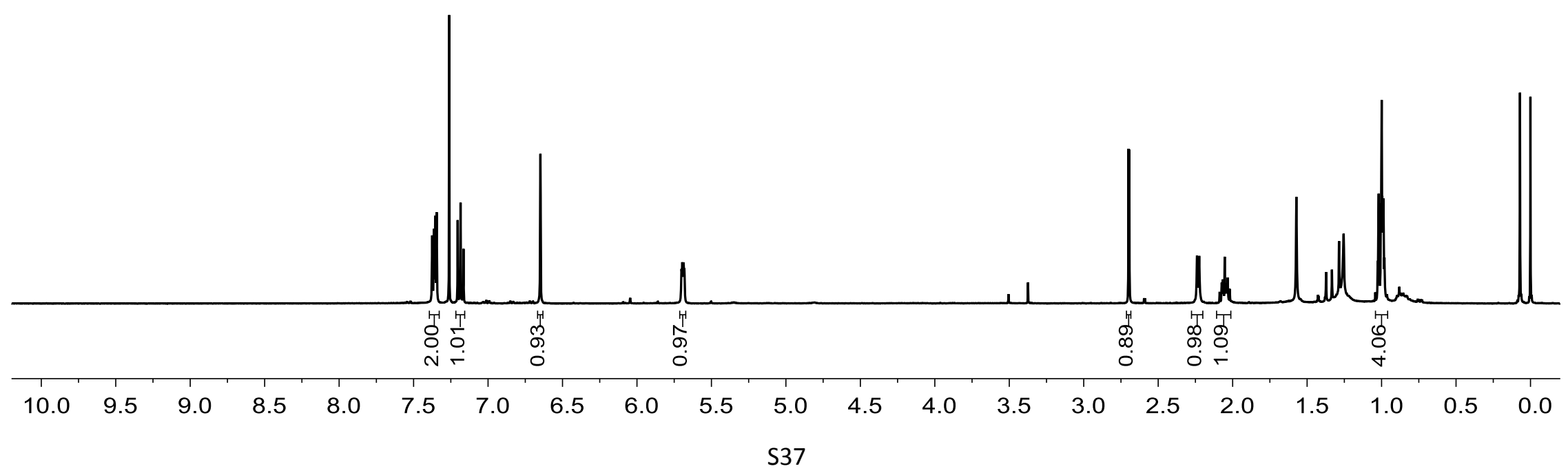




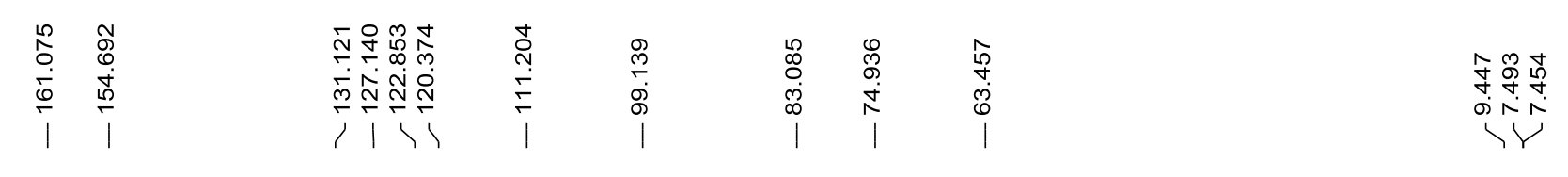

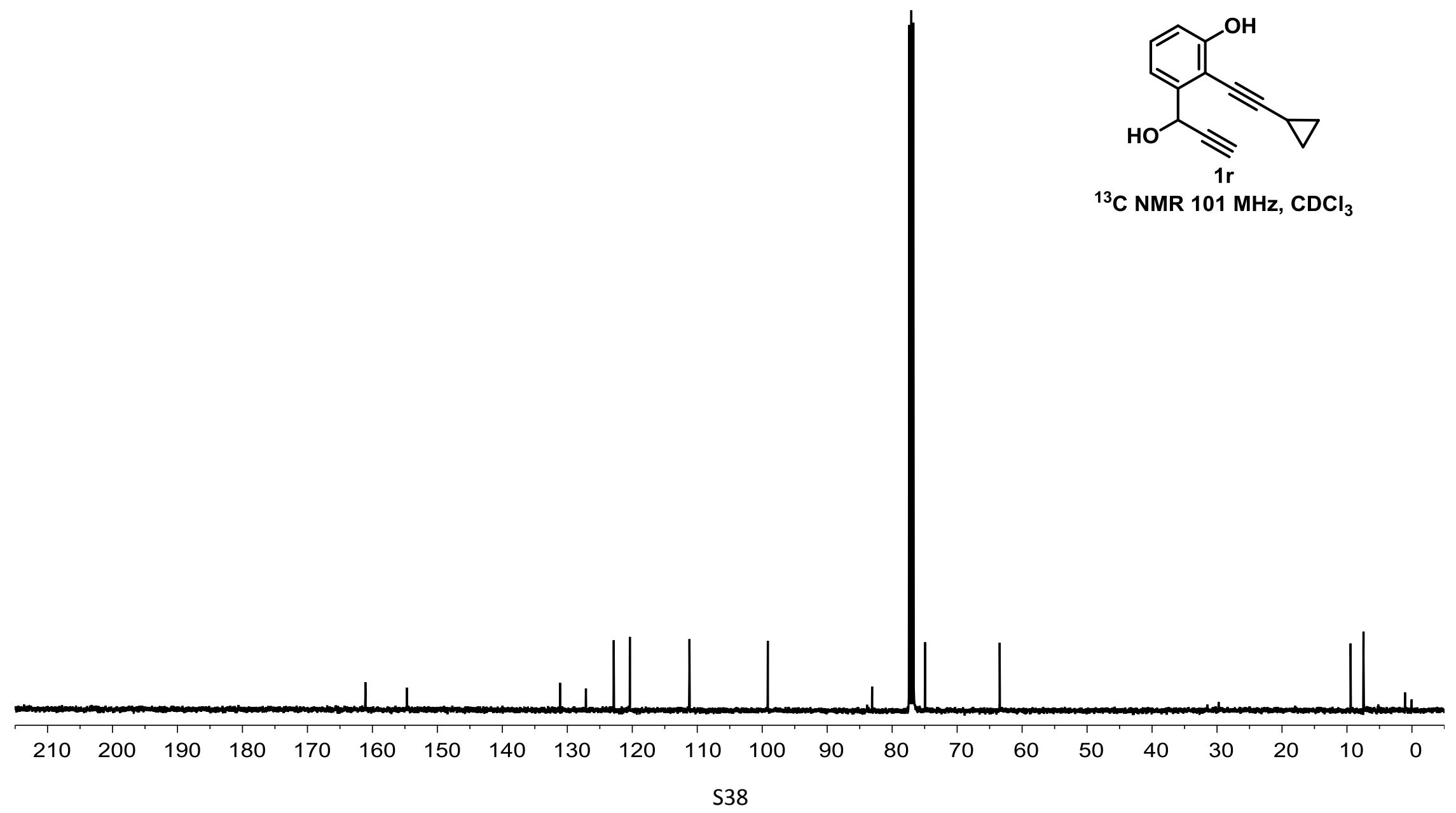



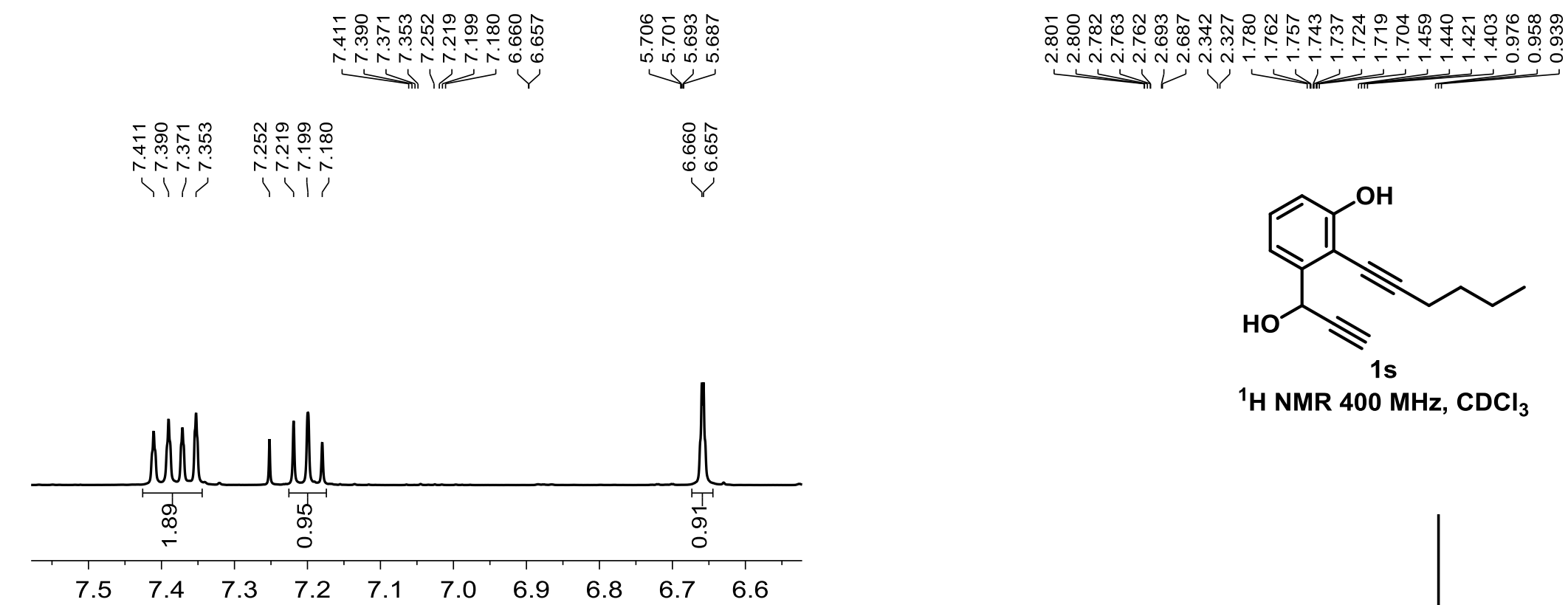

年
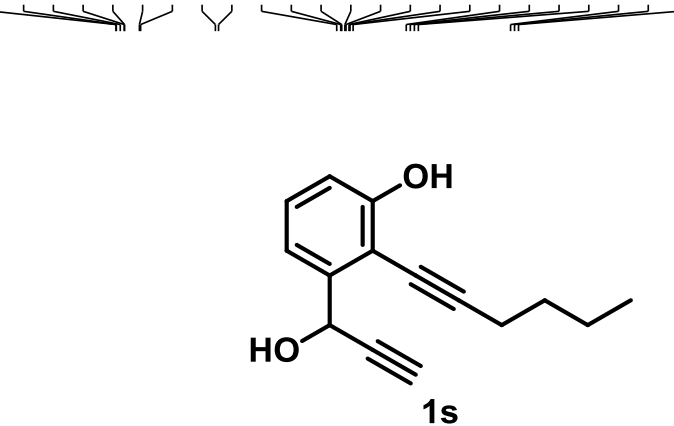

${ }^{1} \mathrm{H}$ NMR $400 \mathrm{MHz}, \mathrm{CDCl}_{3}$

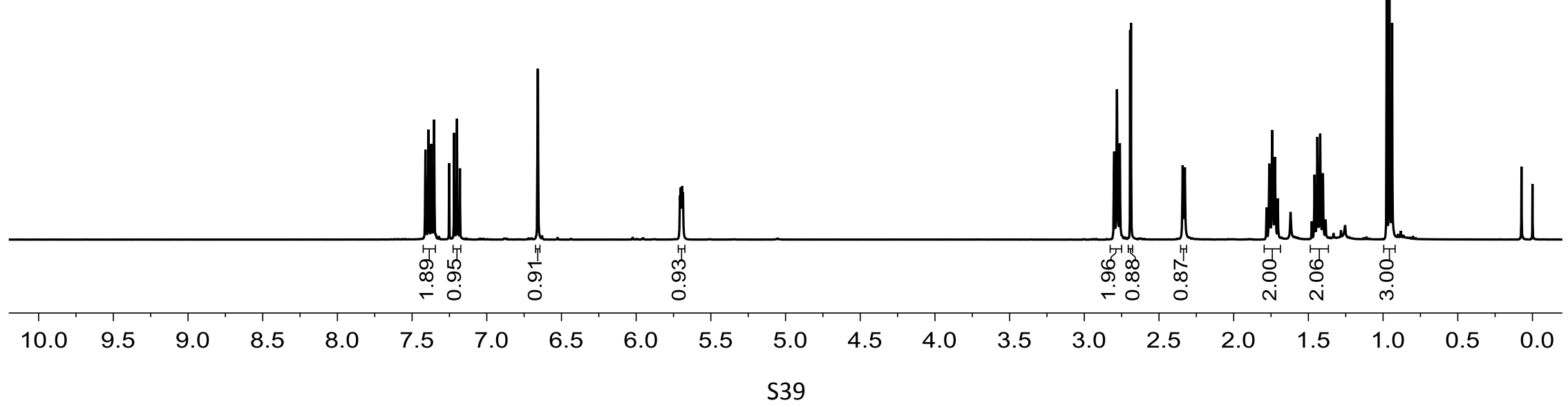




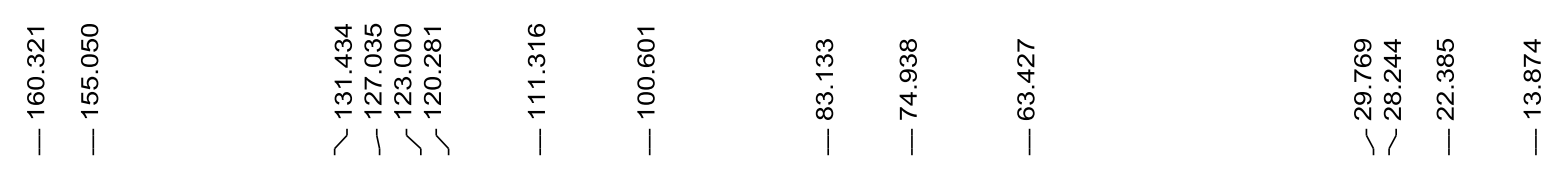

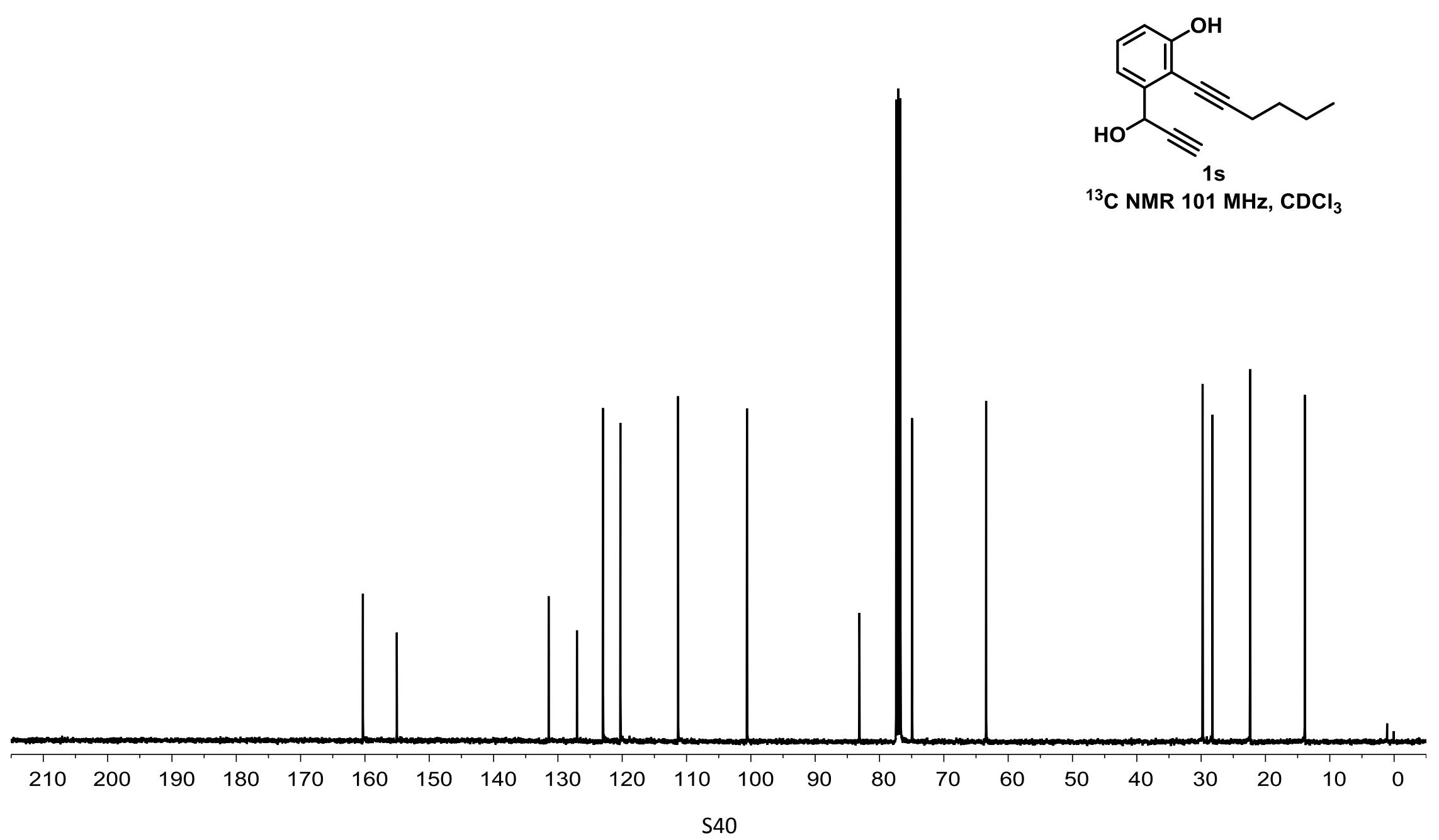




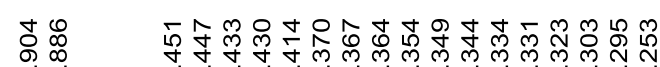

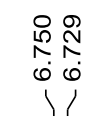
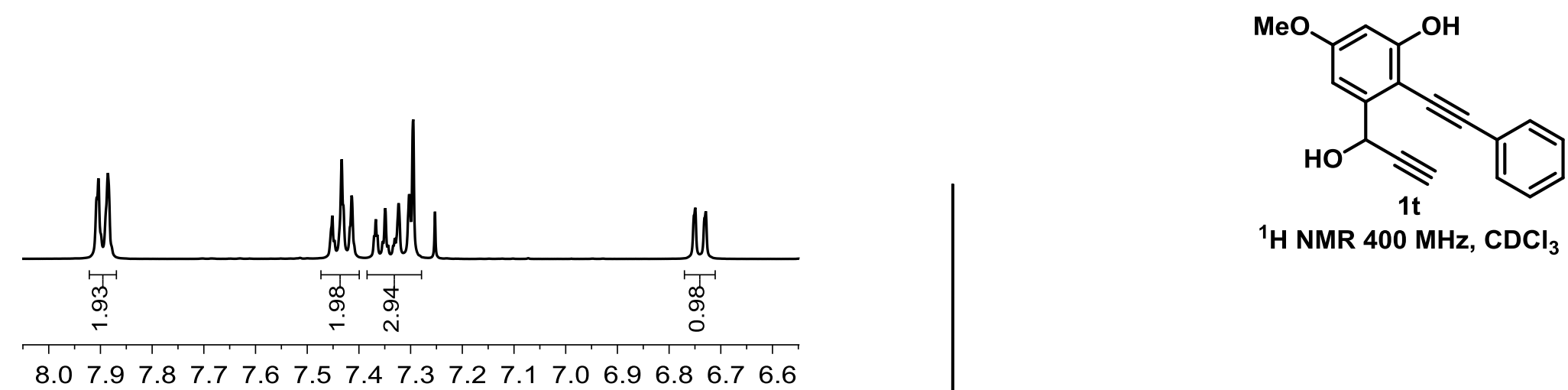

${ }^{1} \mathrm{H}$ NMR $400 \mathrm{MHz}, \mathrm{CDCl}_{3}$

8.07 .97 .87 .77 .67 .57 .47 .37 .27 .17 .06 .96 .86 .76 .6

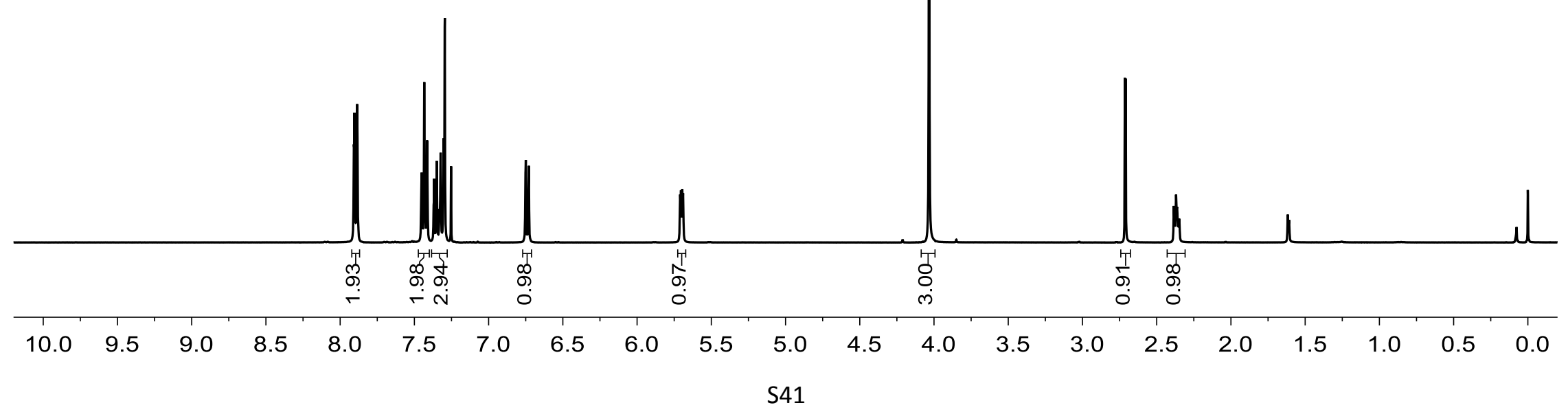




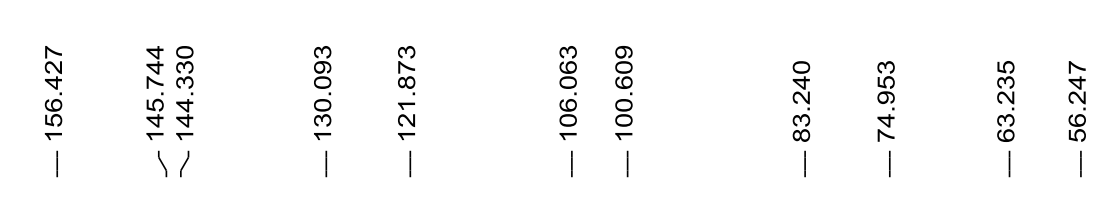

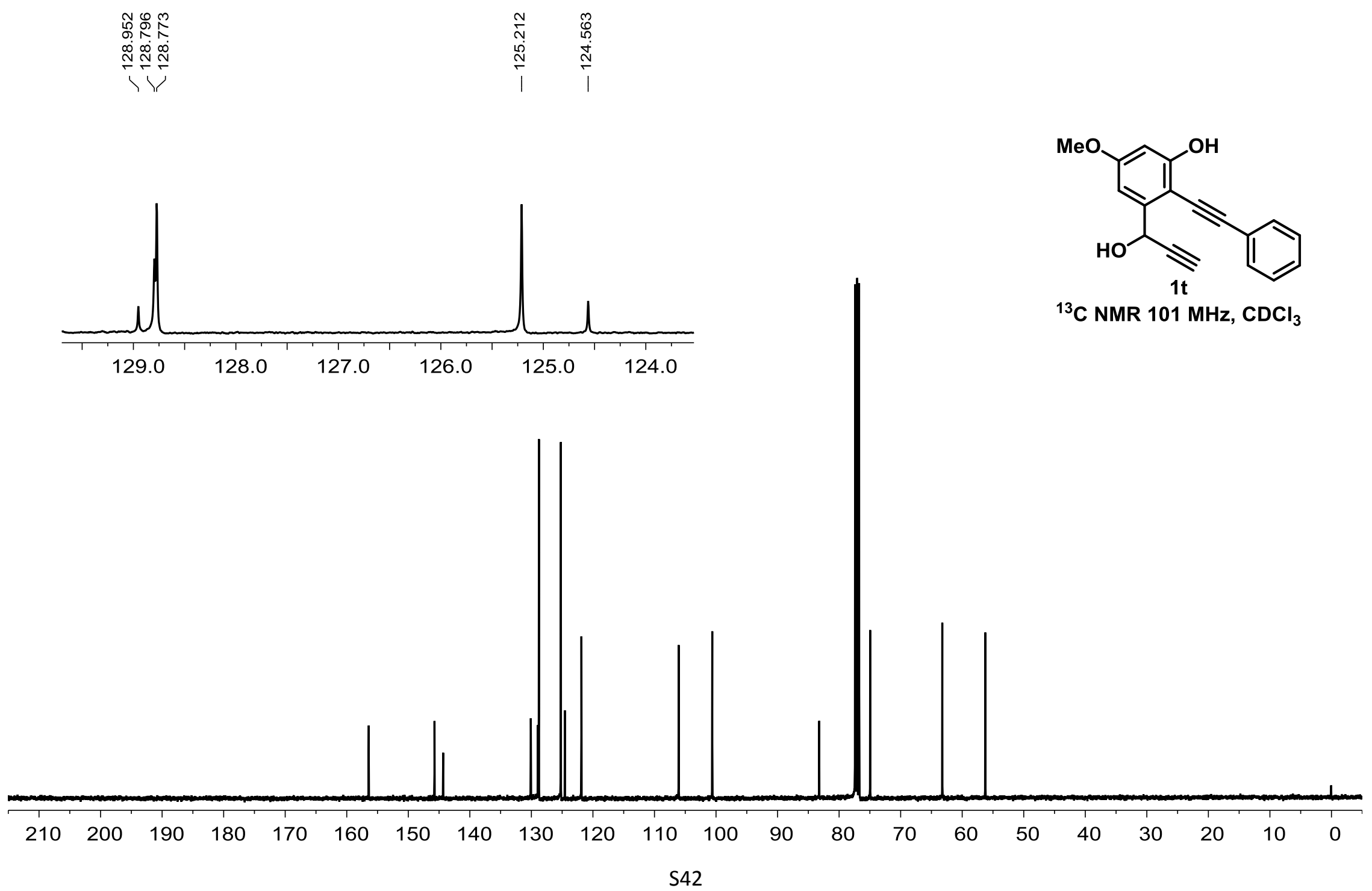




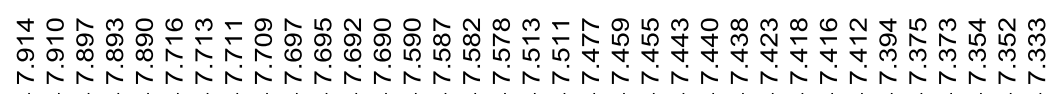

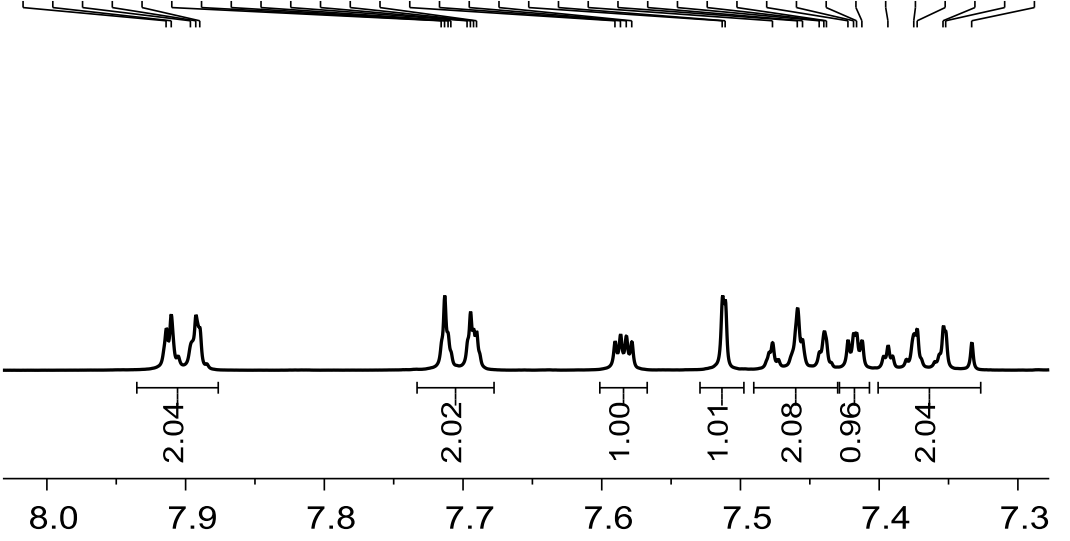

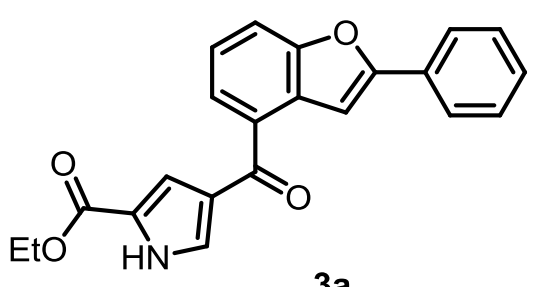

${ }^{1} \mathrm{H}$ NMR $400 \mathrm{MHz}, \mathrm{CDCl}_{3}$

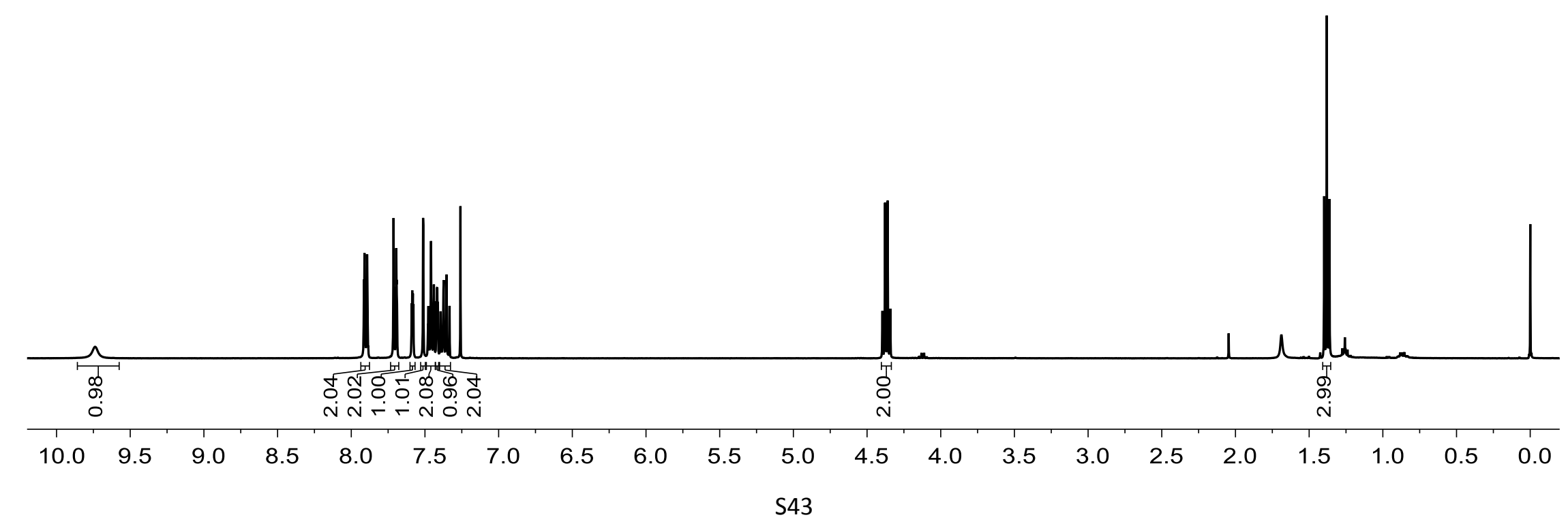




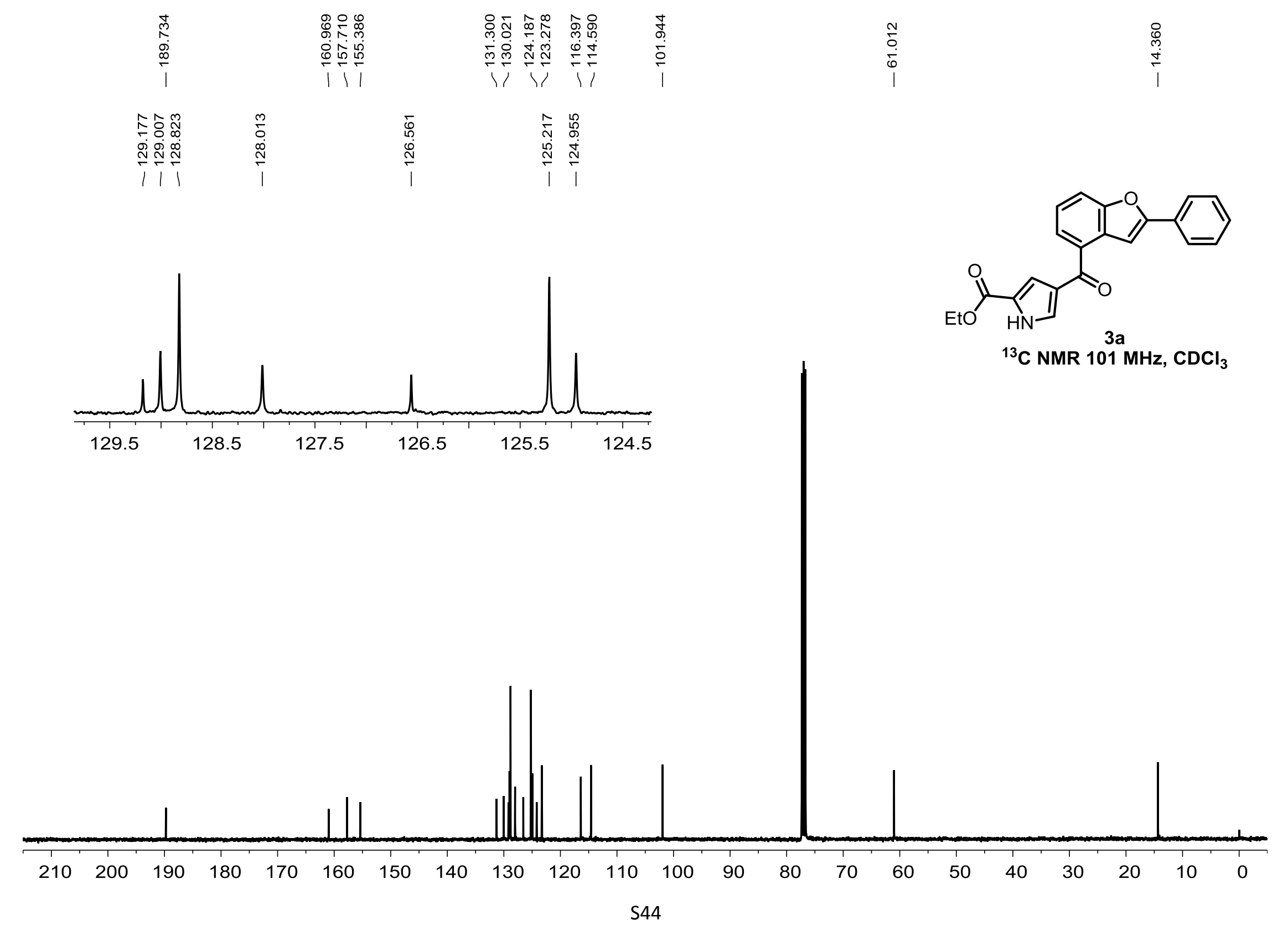


il

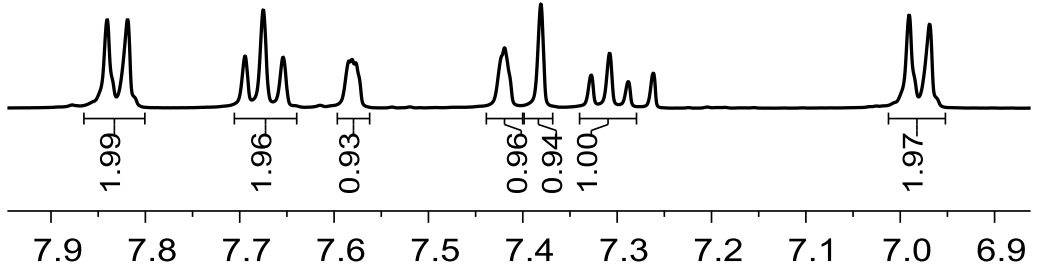

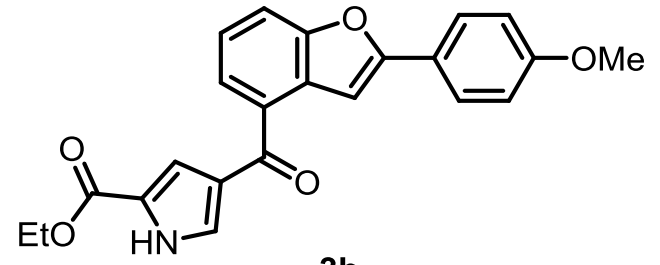

${ }^{1} \mathrm{H}$ NMR $400 \mathrm{MHz}, \mathrm{CDCl}_{3}$

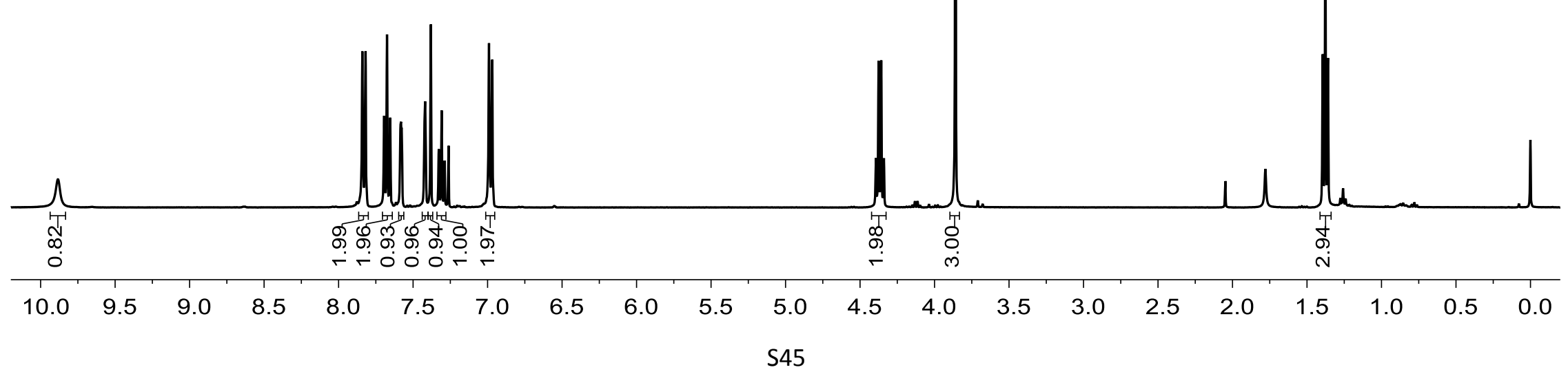




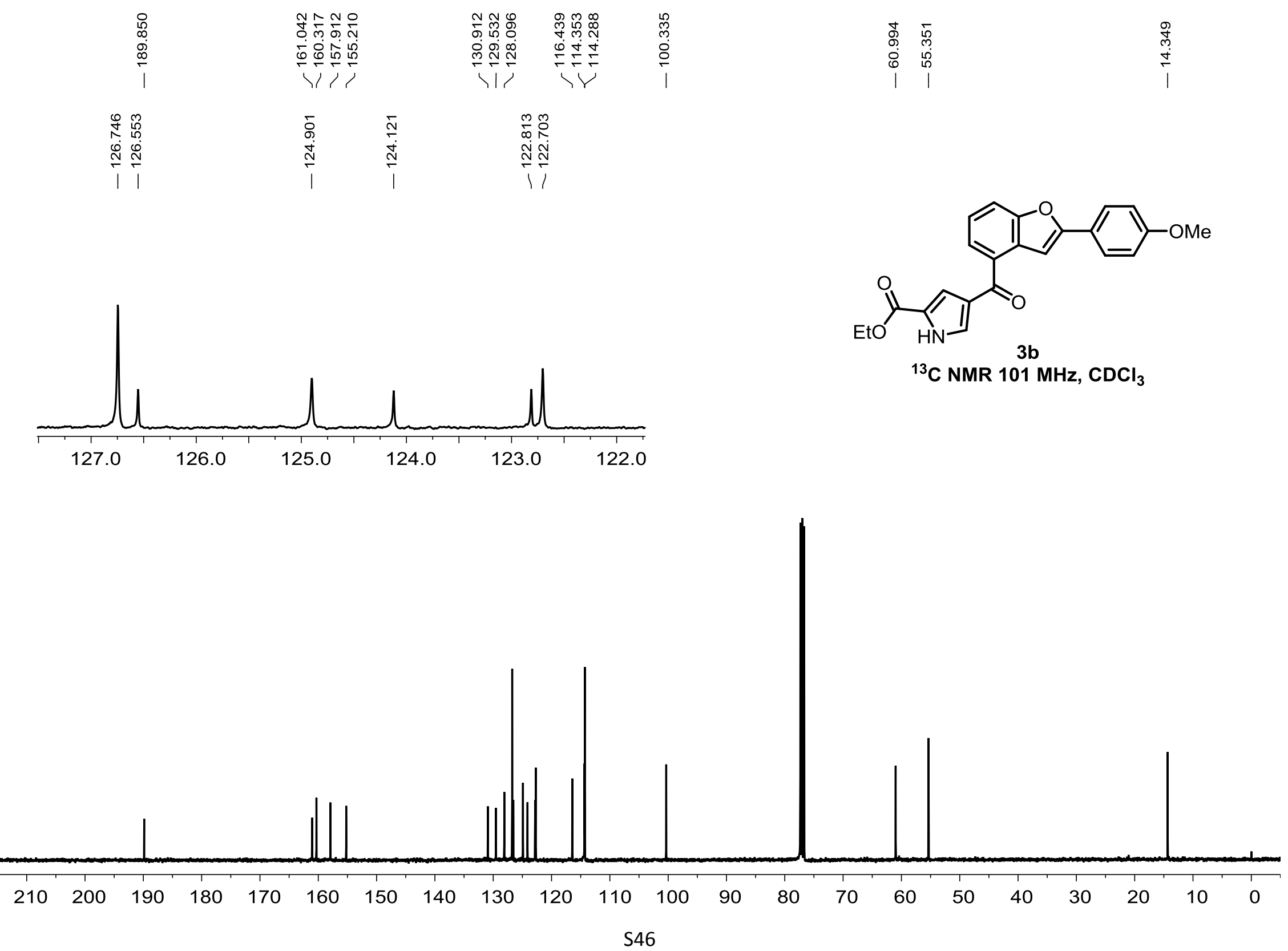


|

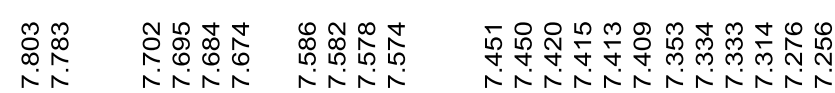

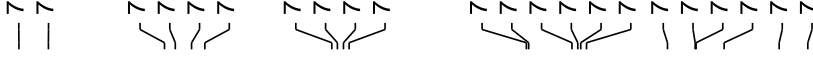

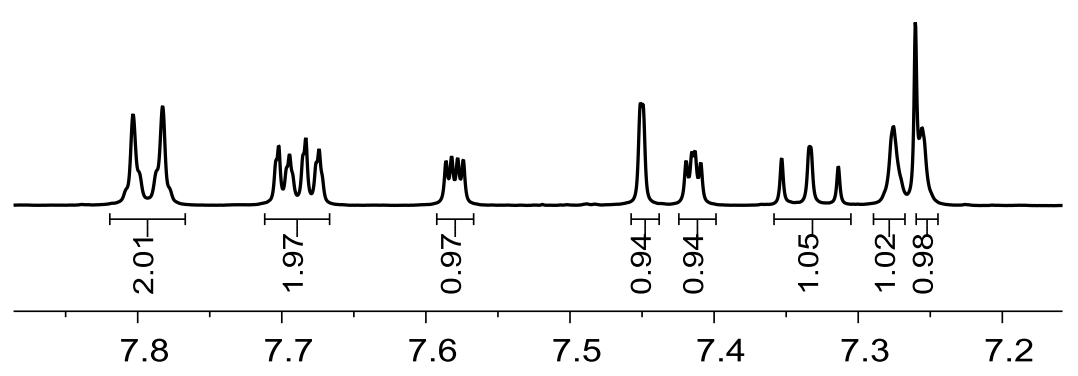

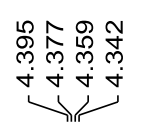

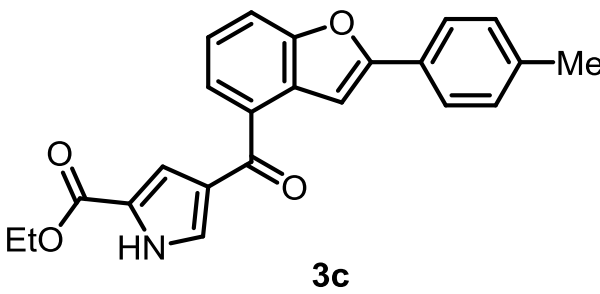

${ }^{1} \mathrm{H}$ NMR $400 \mathrm{MHz}, \mathrm{CDCl}_{3}$

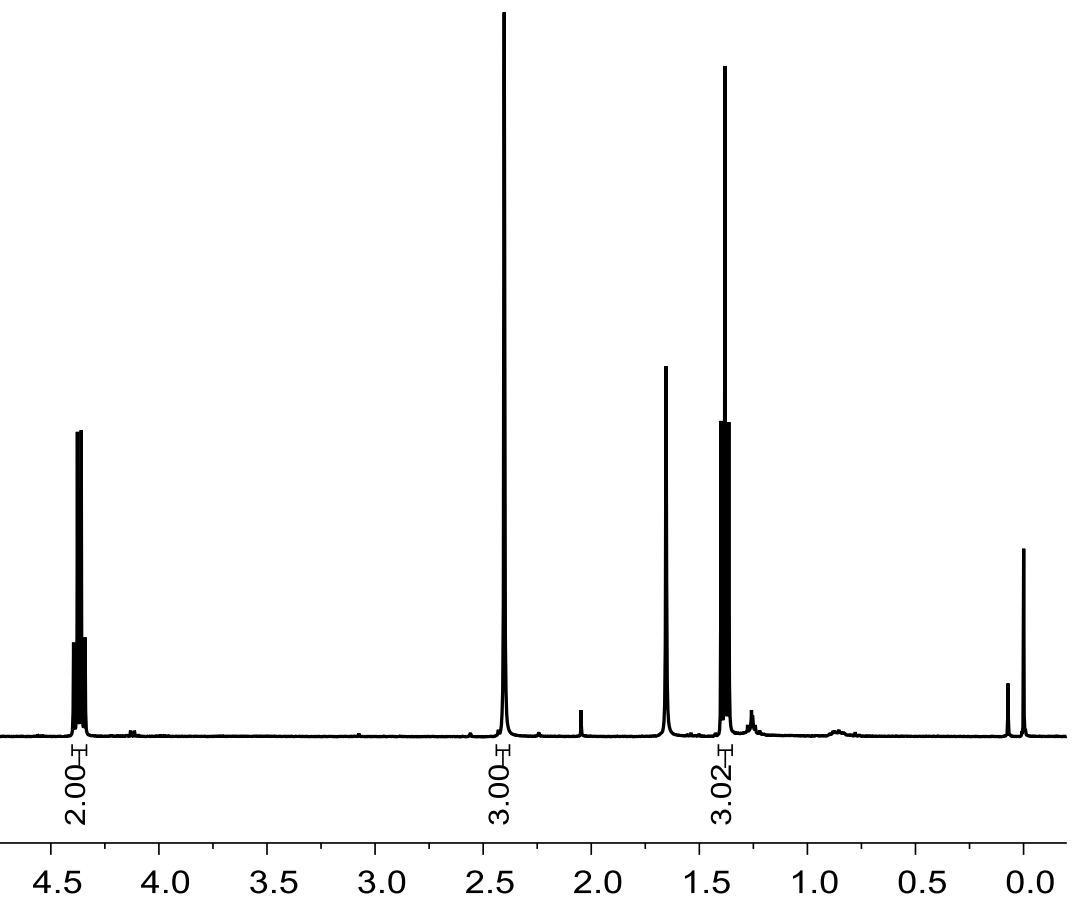




\begin{tabular}{|c|c|c|c|c|c|c|}
\hline g & & & 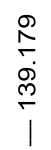 & 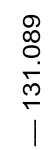 & 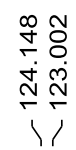 & 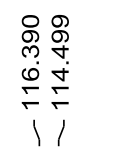 \\
\hline $\begin{array}{l}\text { Oे } \\
\text { 岕 } \\
\stackrel{N}{N} \\
\stackrel{N}{N} \\
\stackrel{1}{N}\end{array}$ & $\begin{array}{l}\stackrel{+}{\infty} \\
\stackrel{\circ}{+} \\
\stackrel{N}{\sim}\end{array}$ & 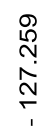 & $\begin{array}{l}\stackrel{9}{1} \\
10 \\
0 \\
\stackrel{0}{\leftarrow} \\
\end{array}$ & & & 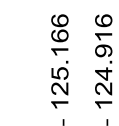 \\
\hline 11 & 1 & 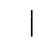 & 1 & & & 11 \\
\hline
\end{tabular}

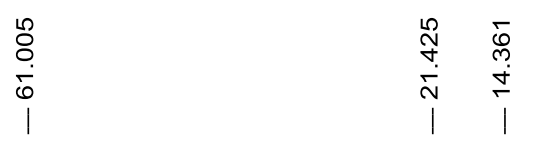
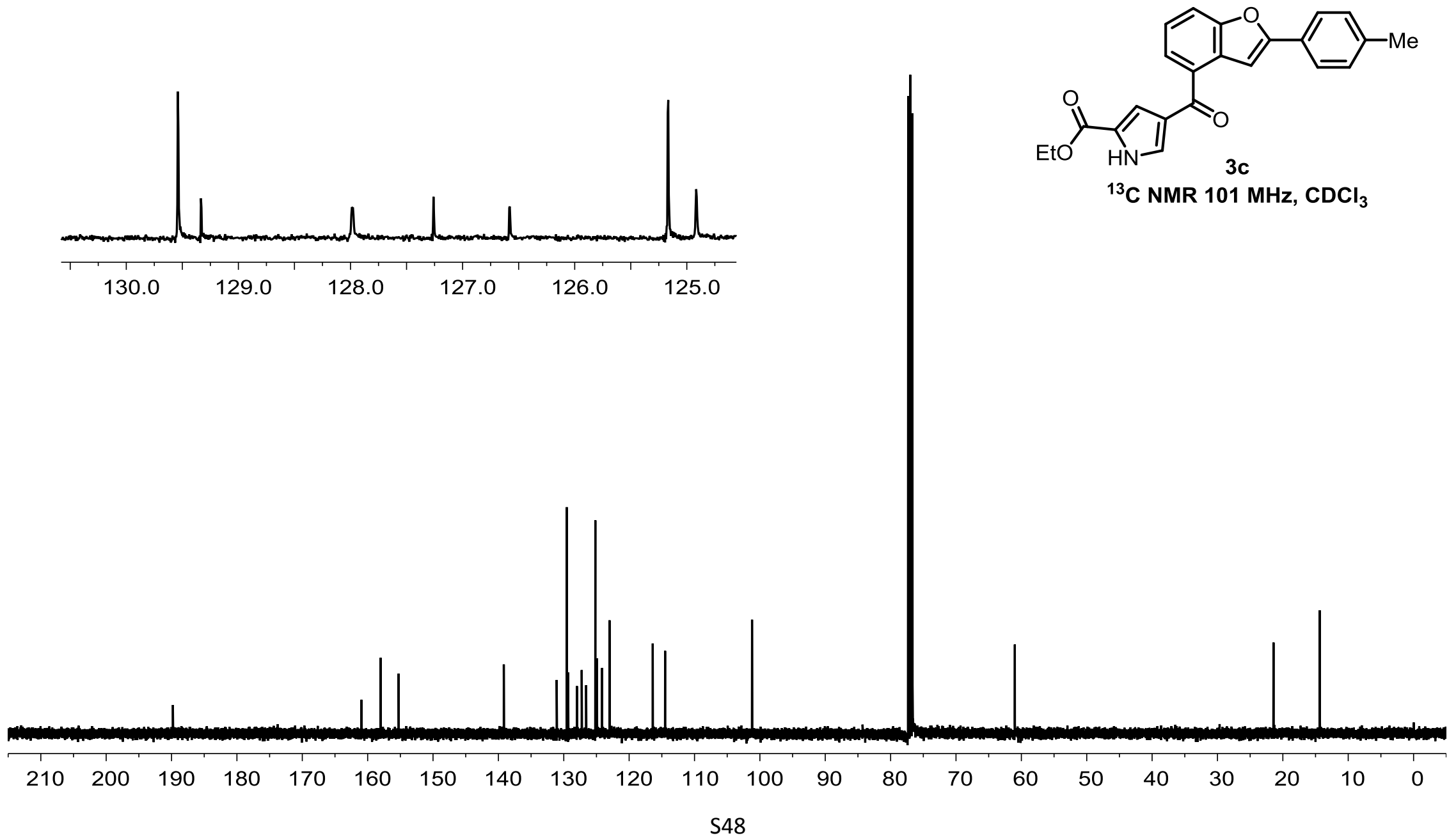


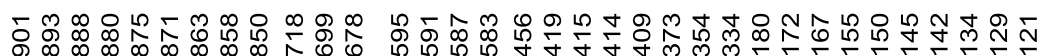

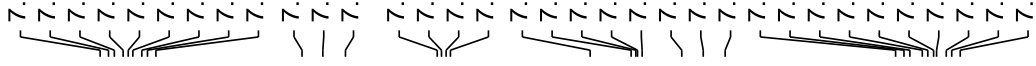
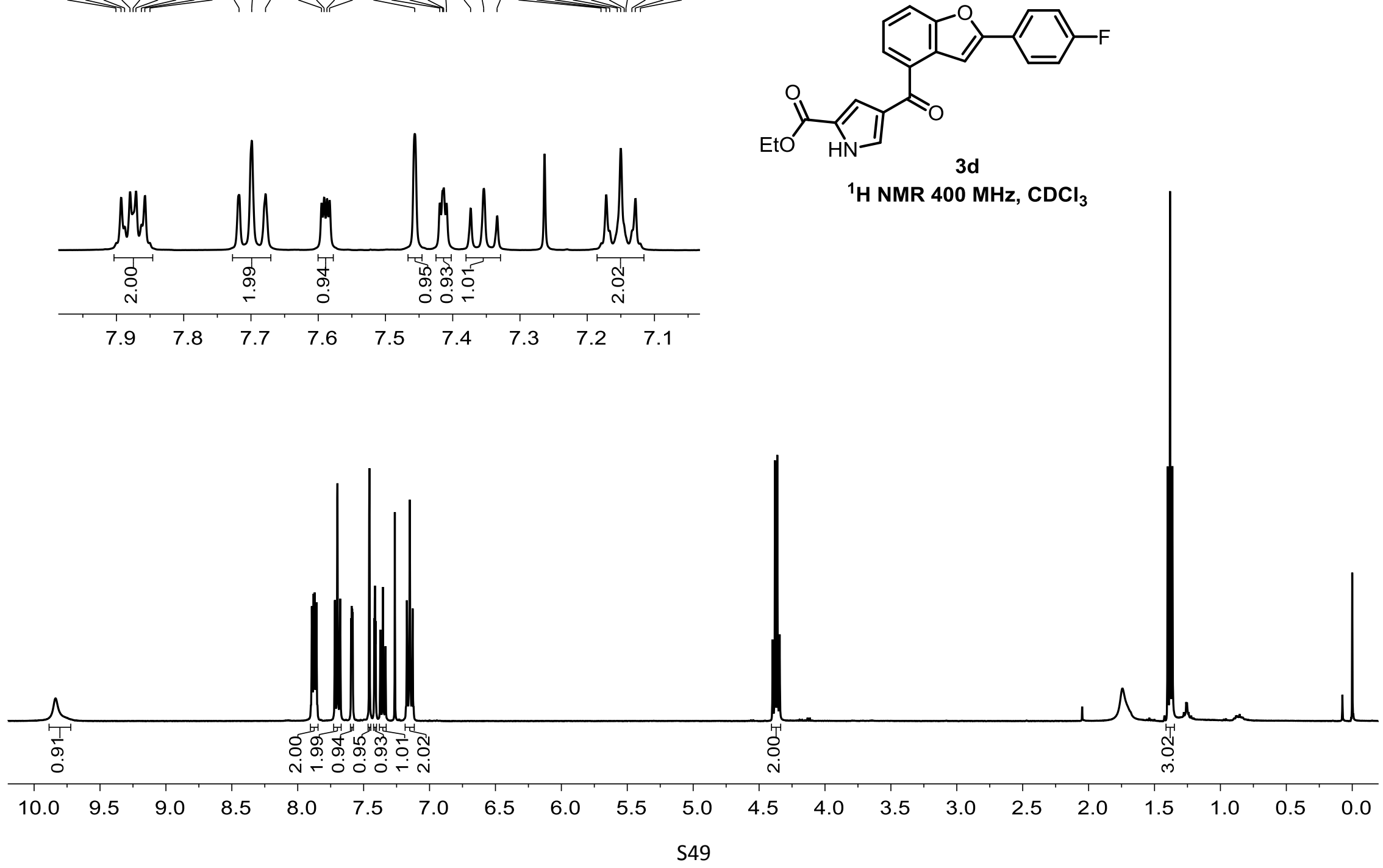


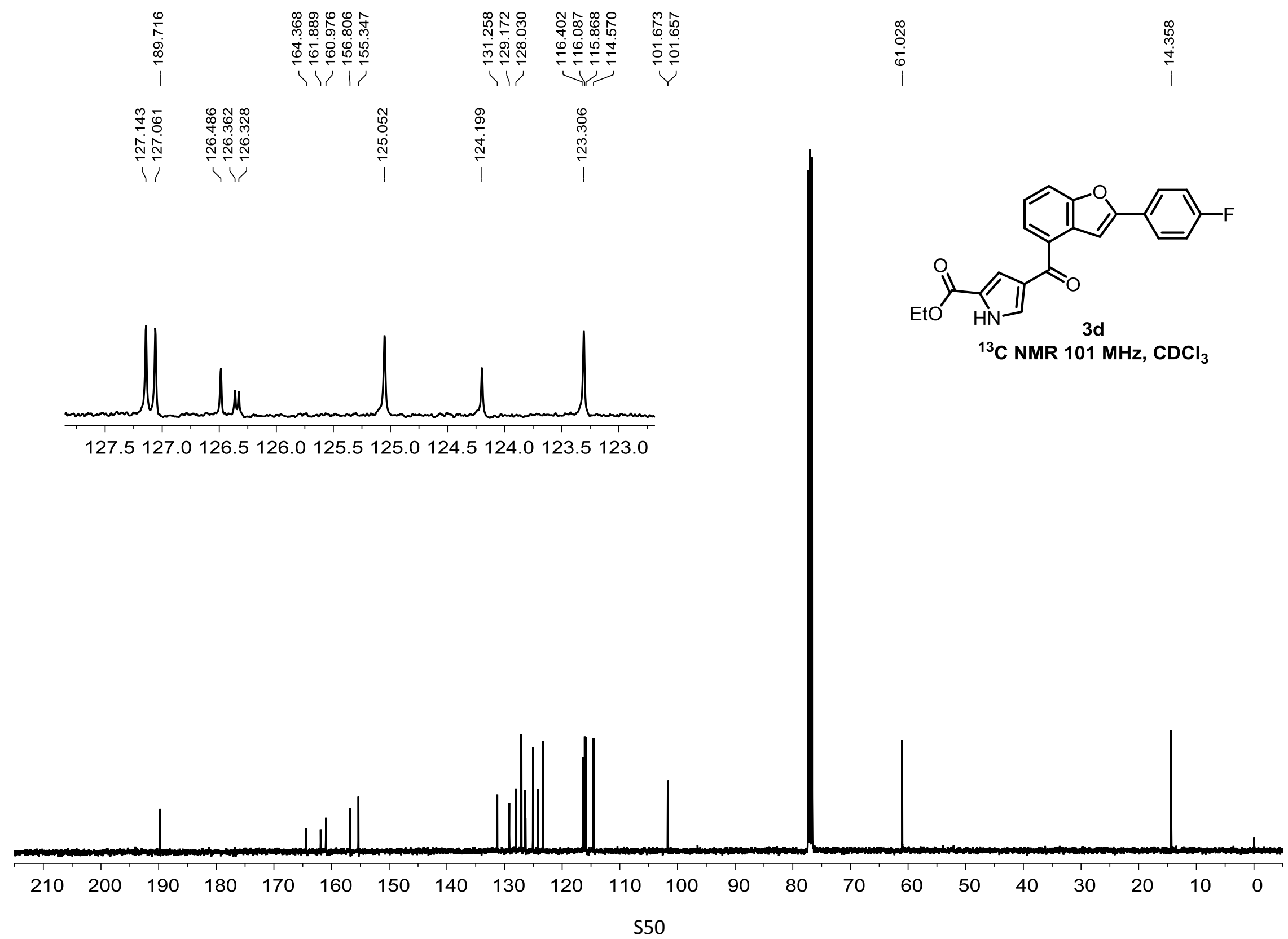




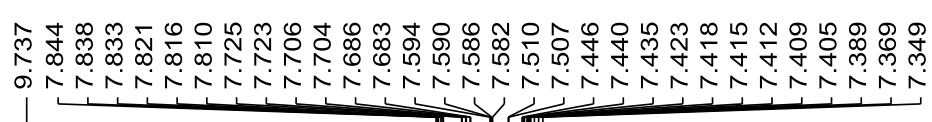

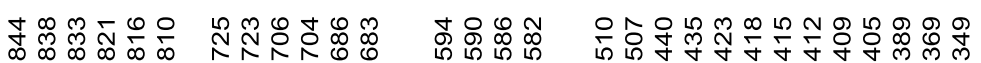

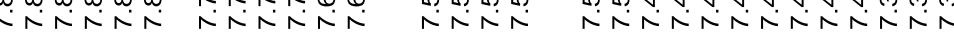

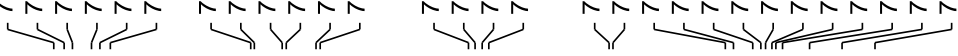

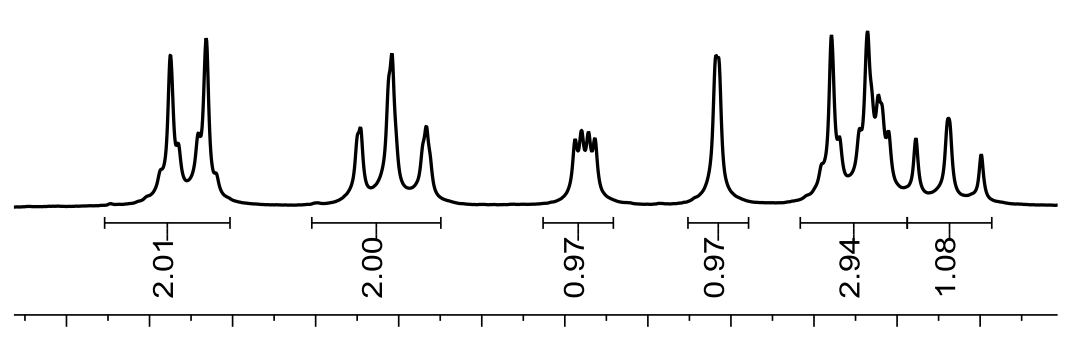

7.907 .857 .807 .757 .707 .657 .607 .557 .507 .457 .407 .35

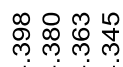

$\dot{4}+\dot{P}$

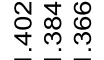

我

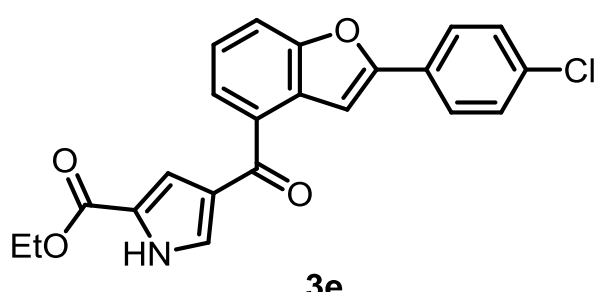

${ }^{1} \mathrm{H}$ NMR $400 \mathrm{MHz}^{\mathrm{CDCl}}$

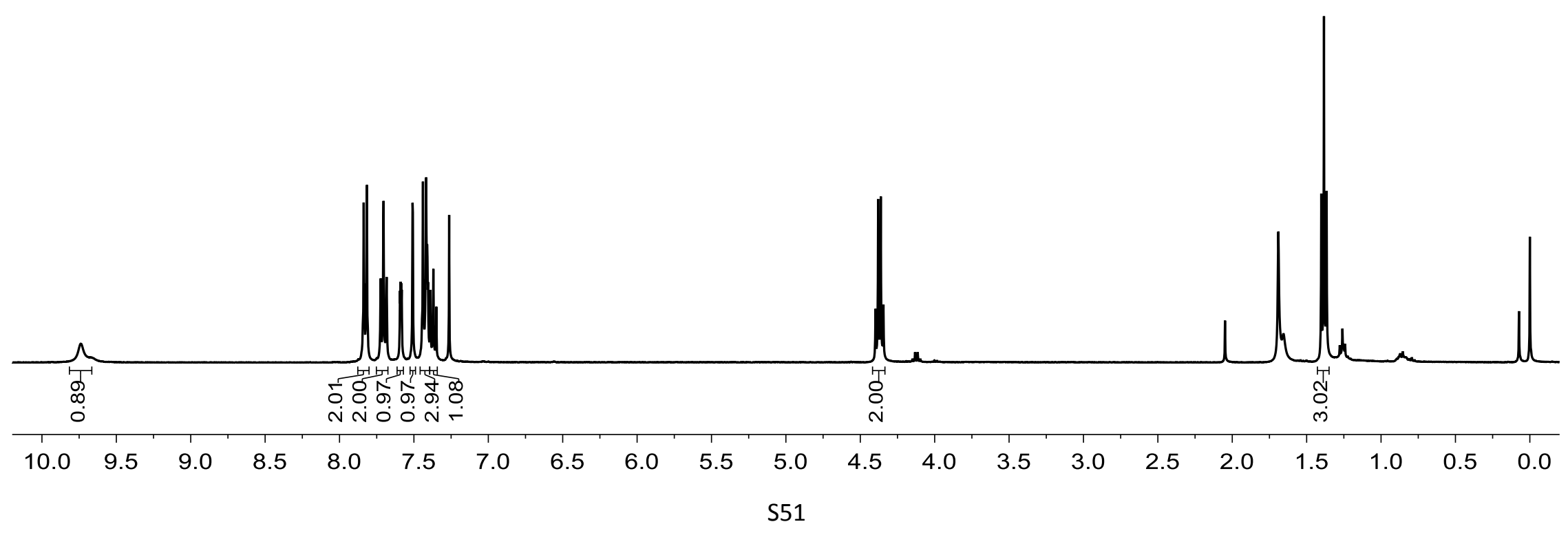




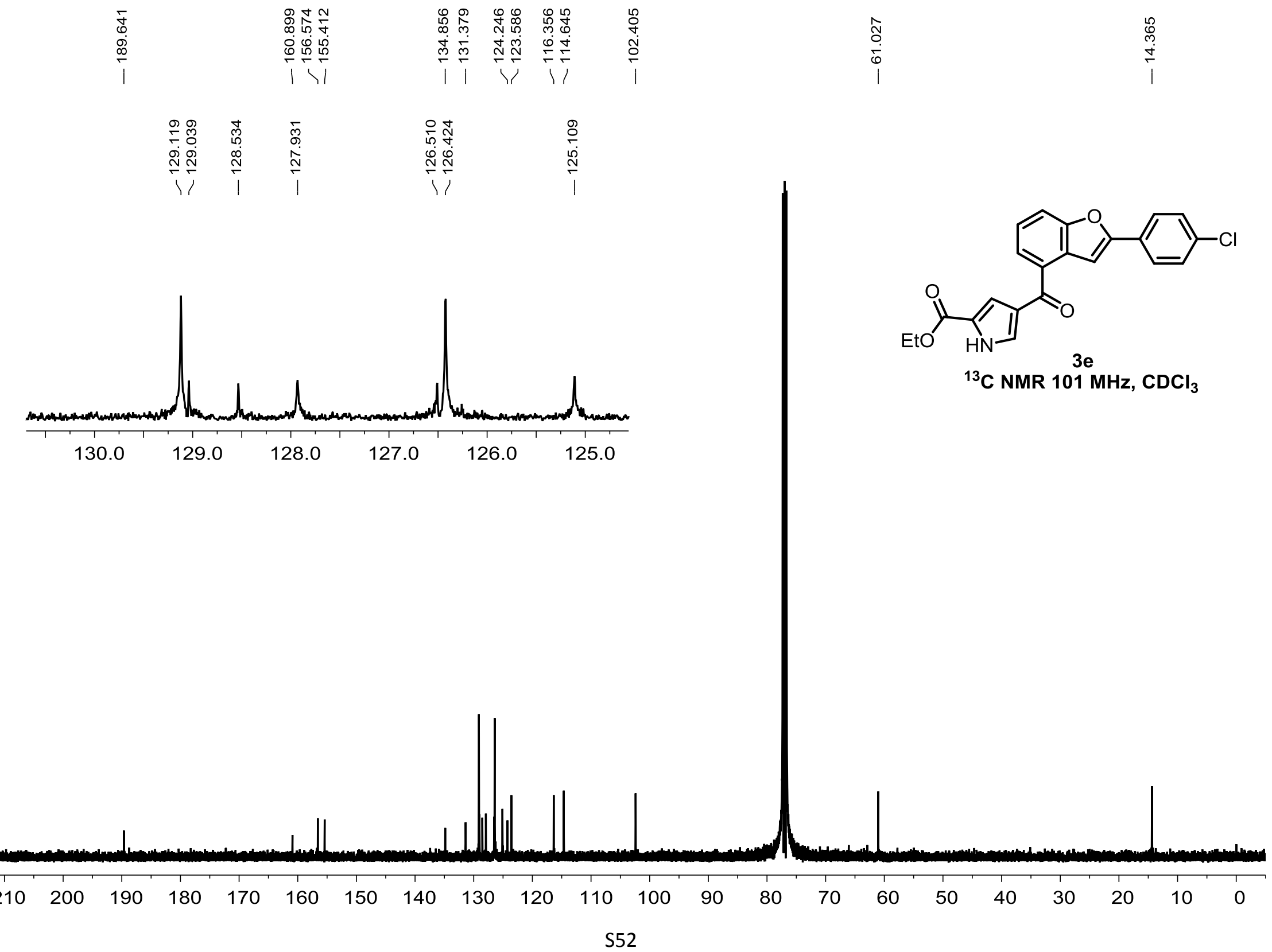




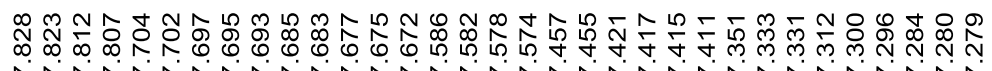
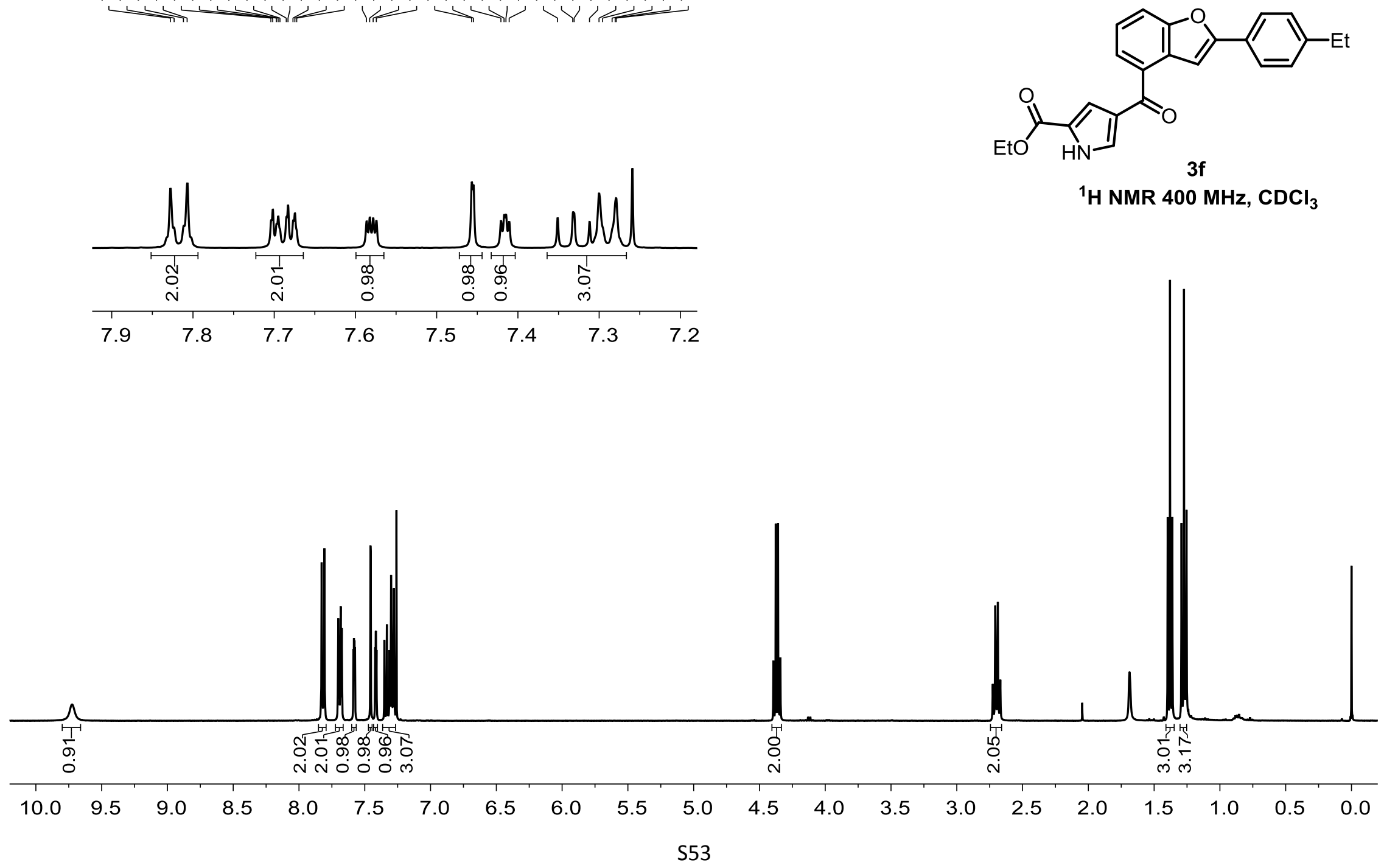


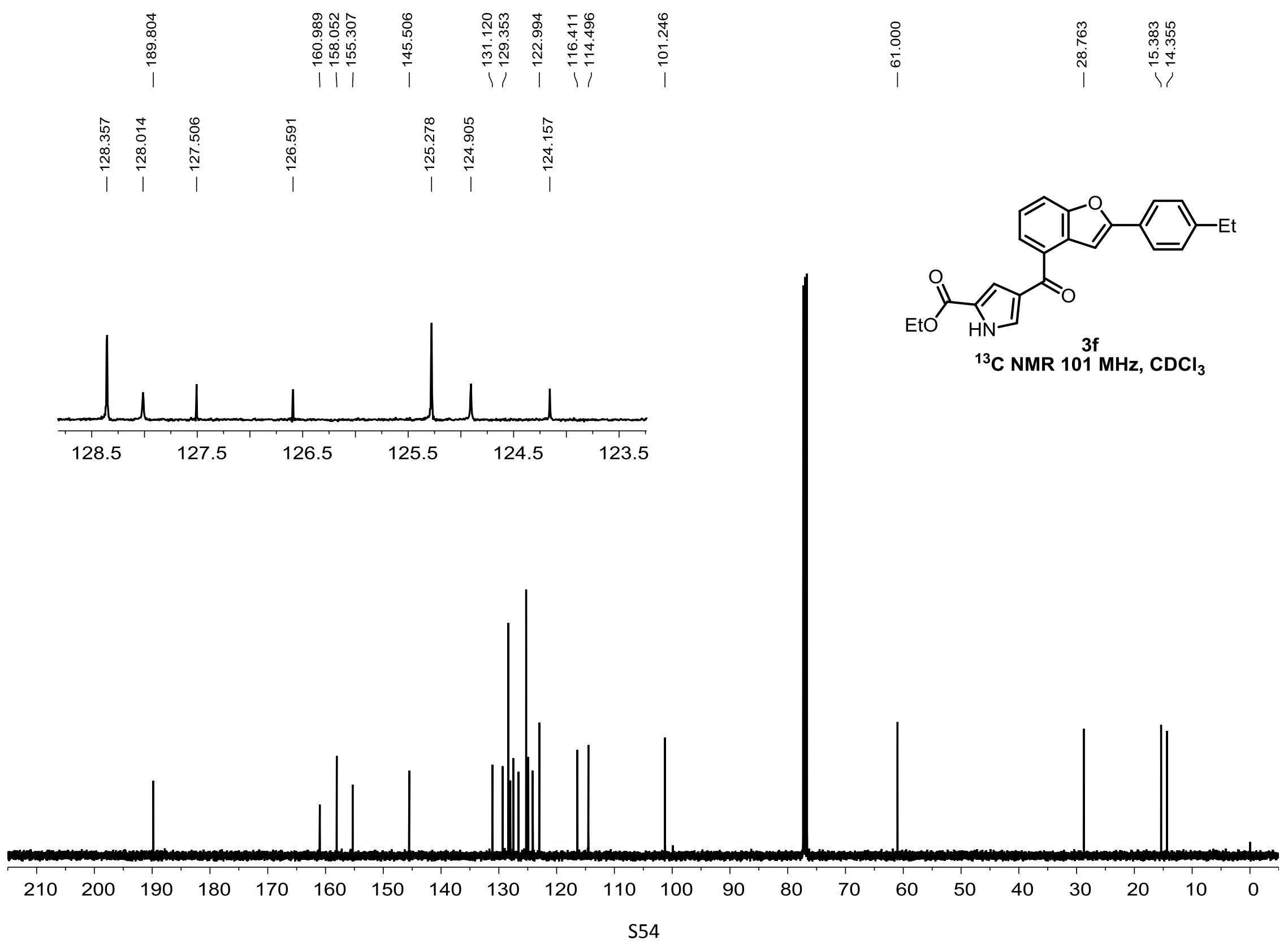




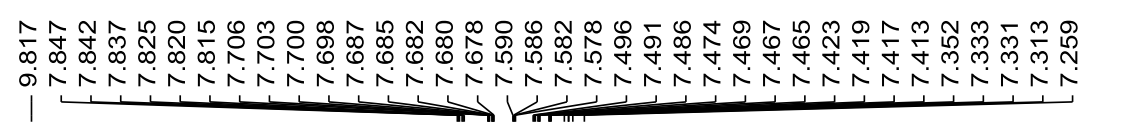

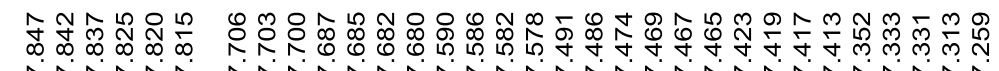

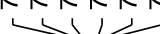
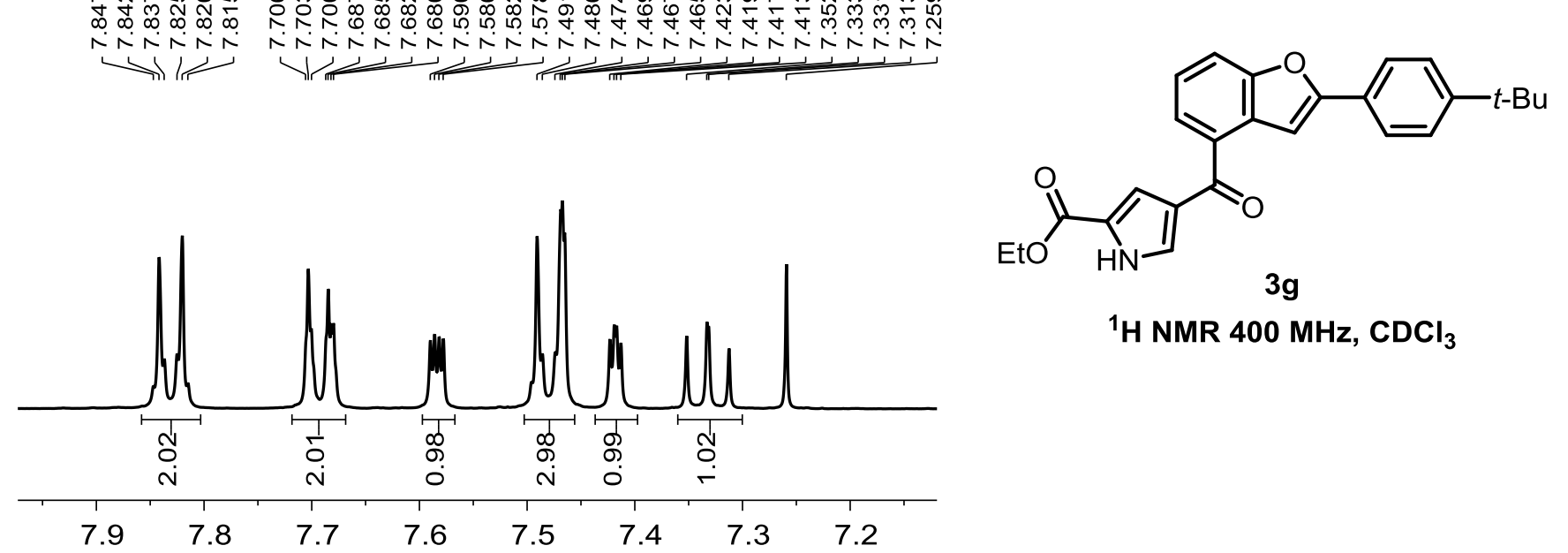

${ }^{1} \mathrm{H}$ NMR $400 \mathrm{MHz}, \mathrm{CDCl}_{3}$

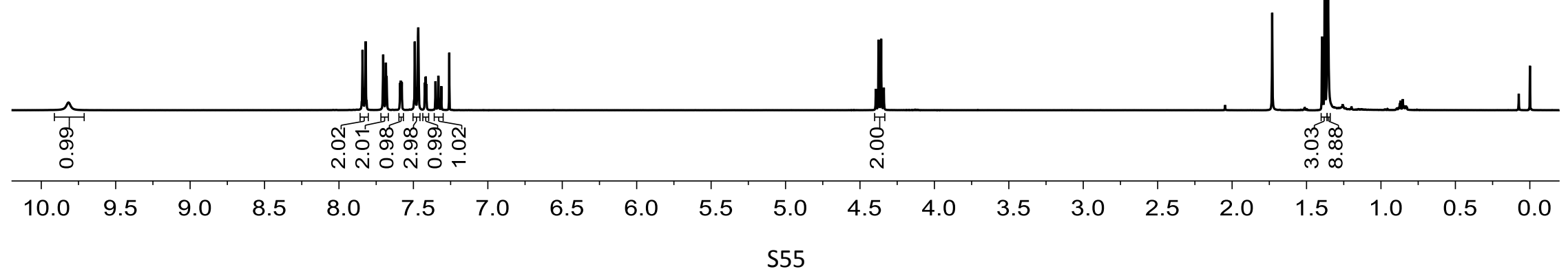




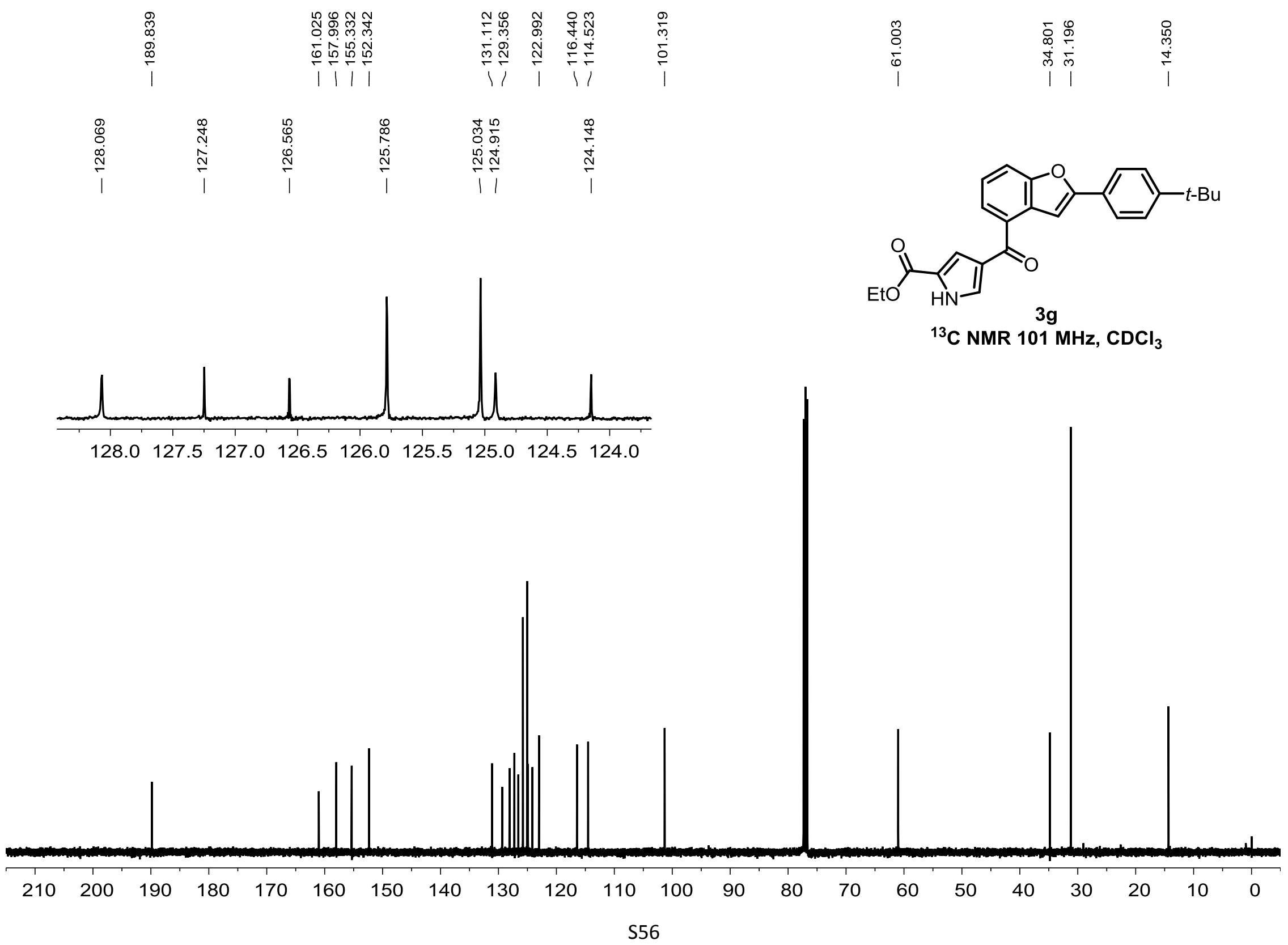




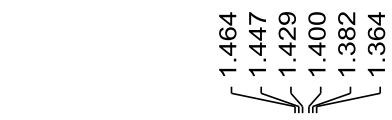

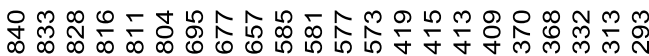

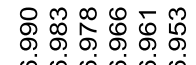

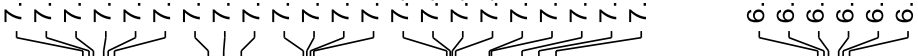
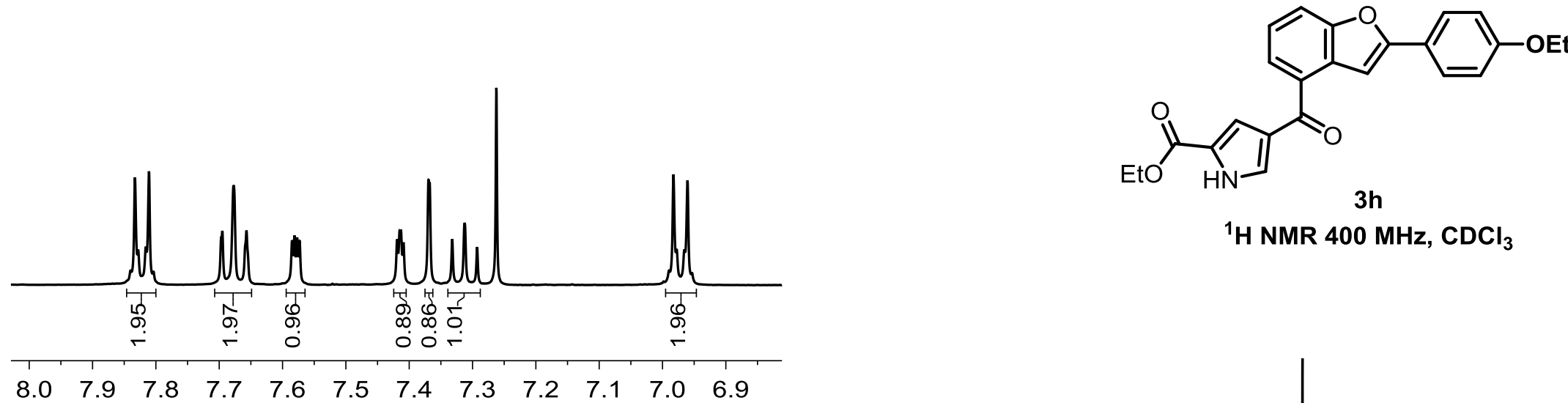

${ }^{1} \mathrm{H}$ NMR $400 \mathrm{MHz}, \mathrm{CDCl}_{3}$

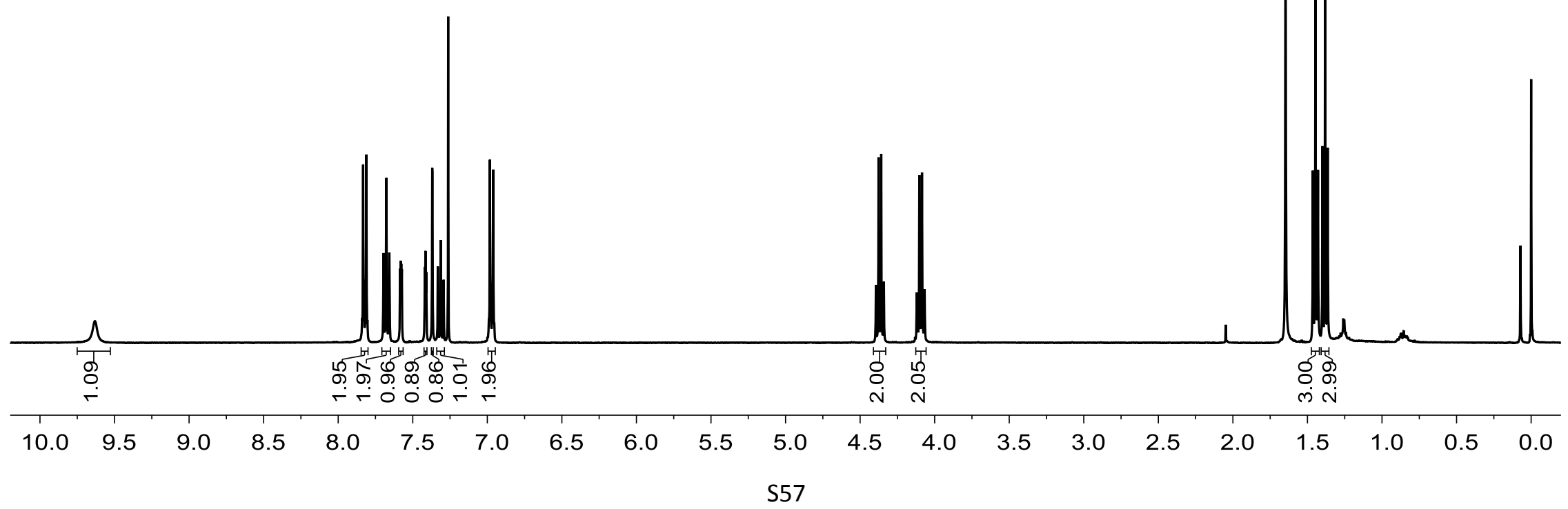




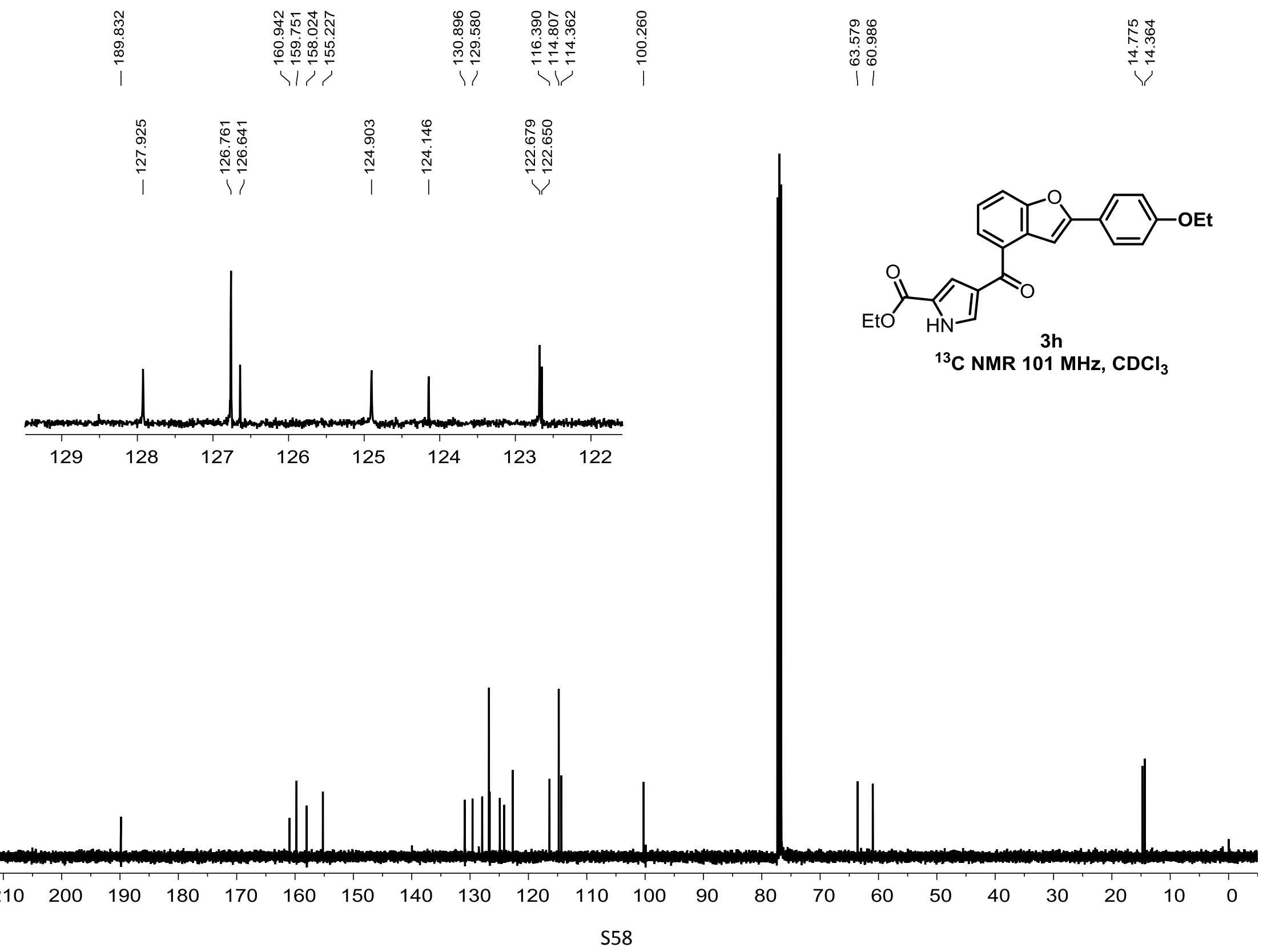




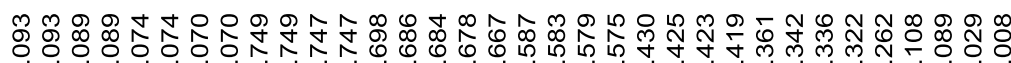
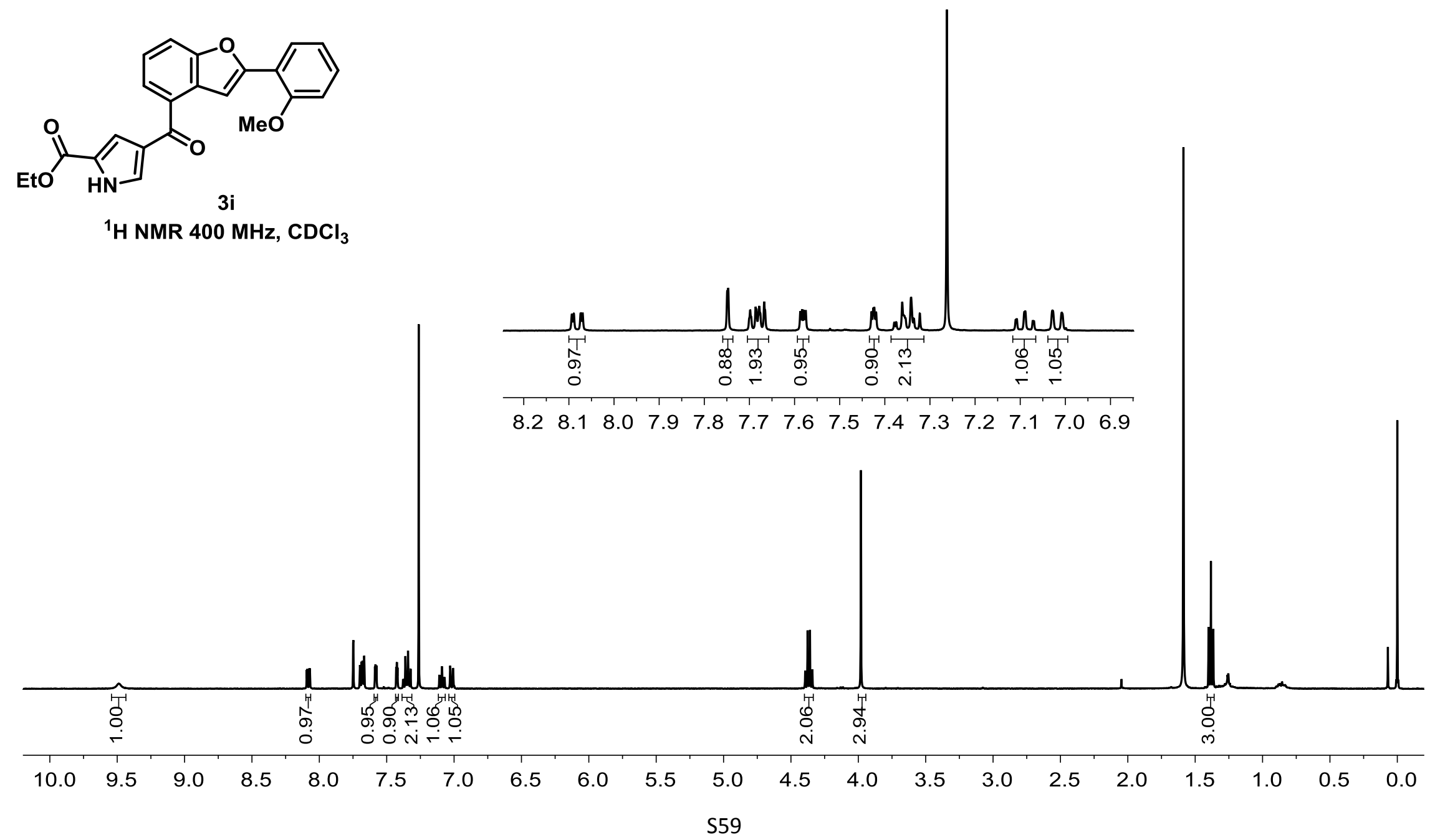


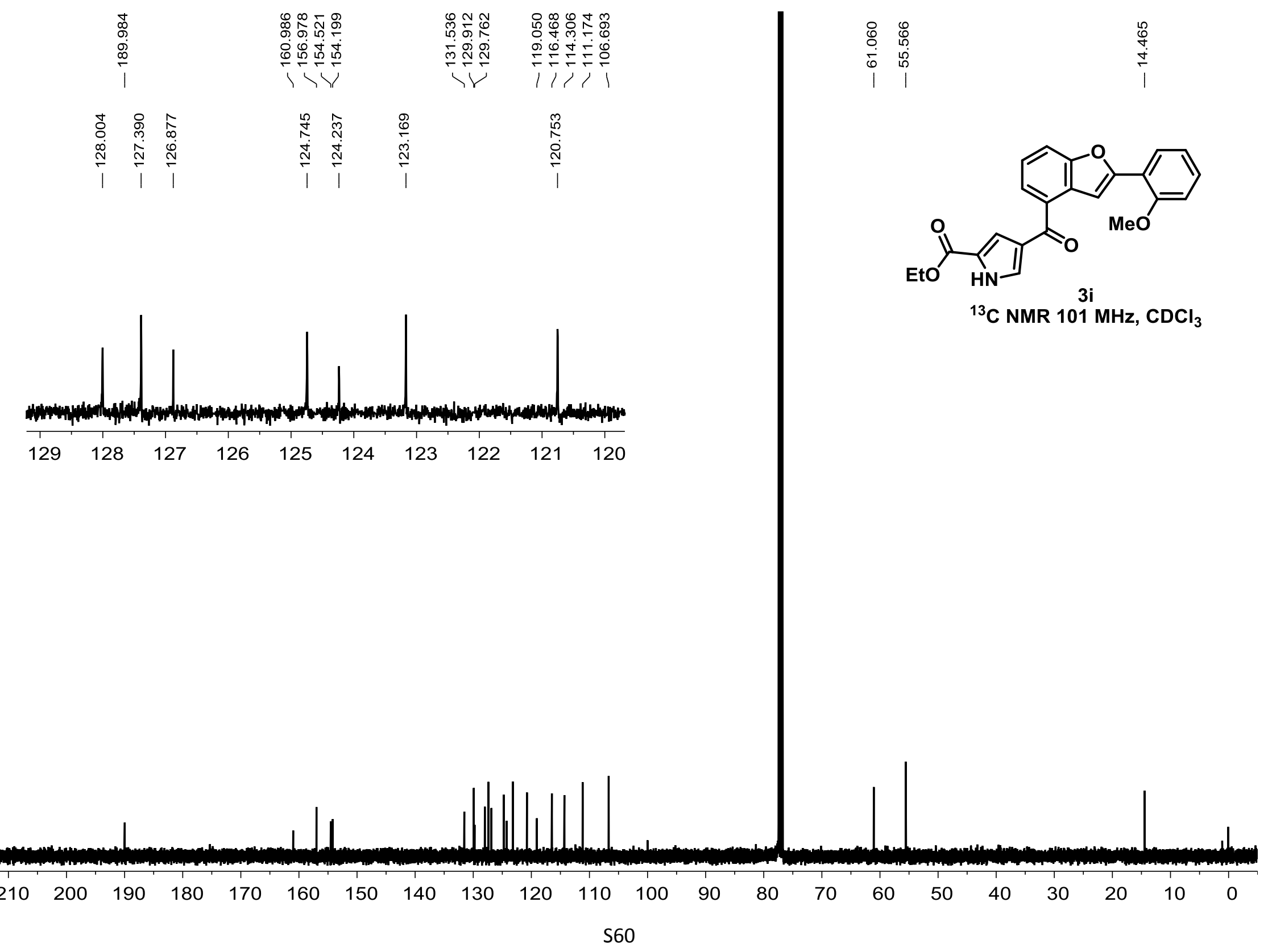




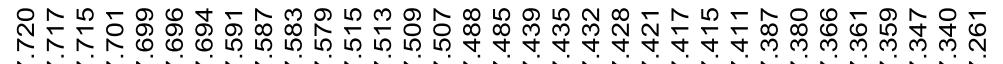
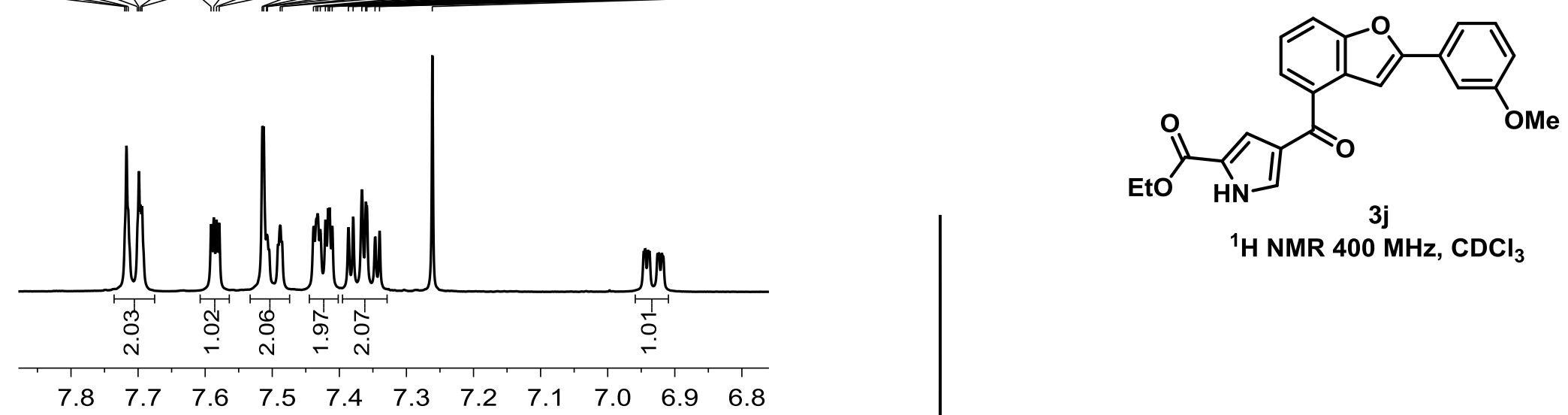

${ }^{1} \mathrm{H}$ NMR $400 \mathrm{MHz}, \mathrm{CDCl}_{3}$

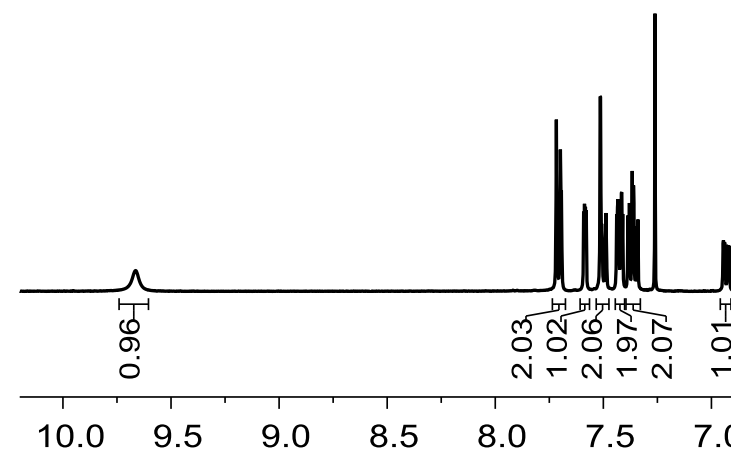

$10.0 \quad 9.5$

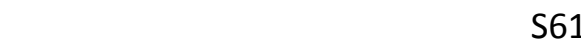




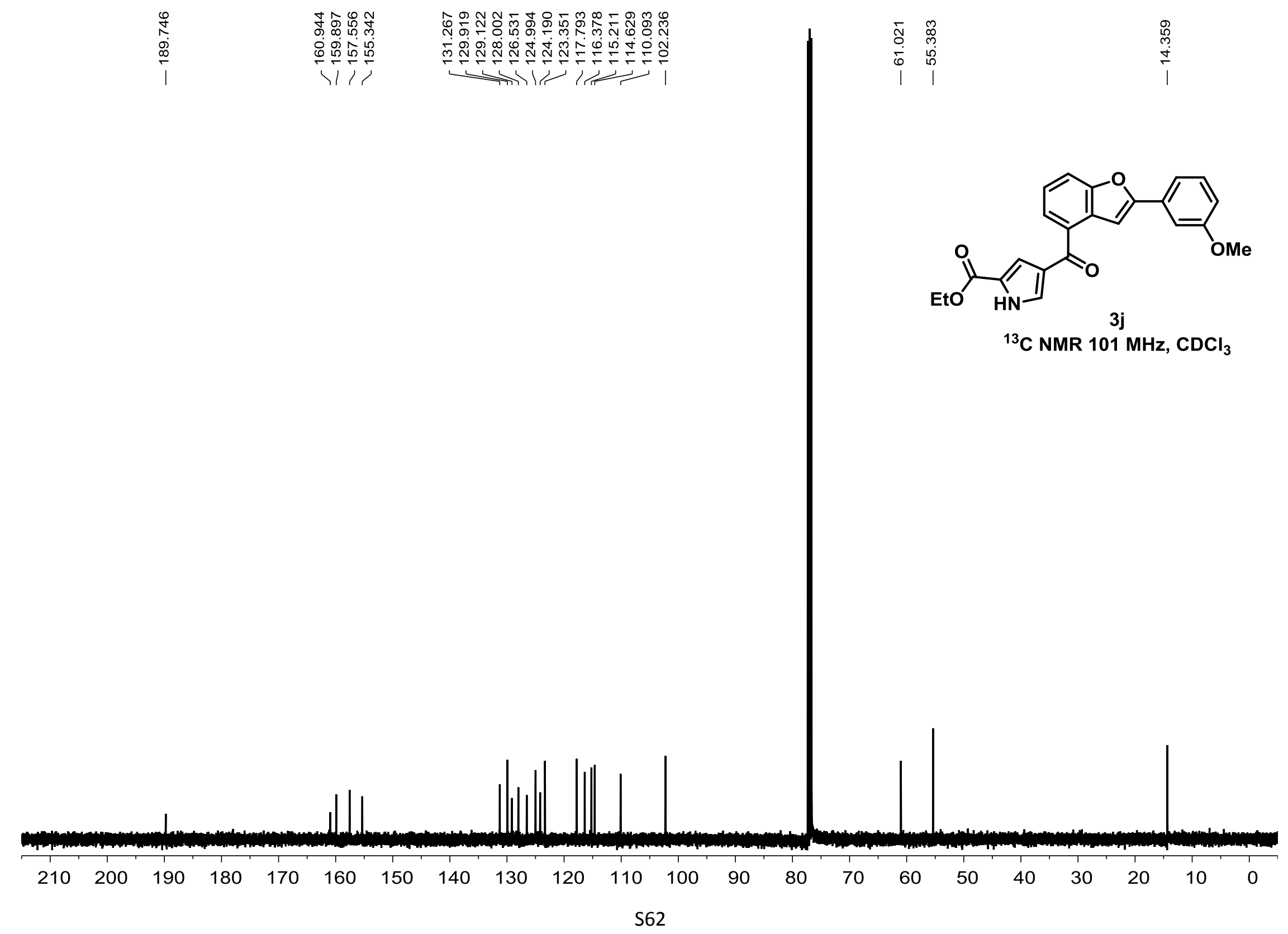




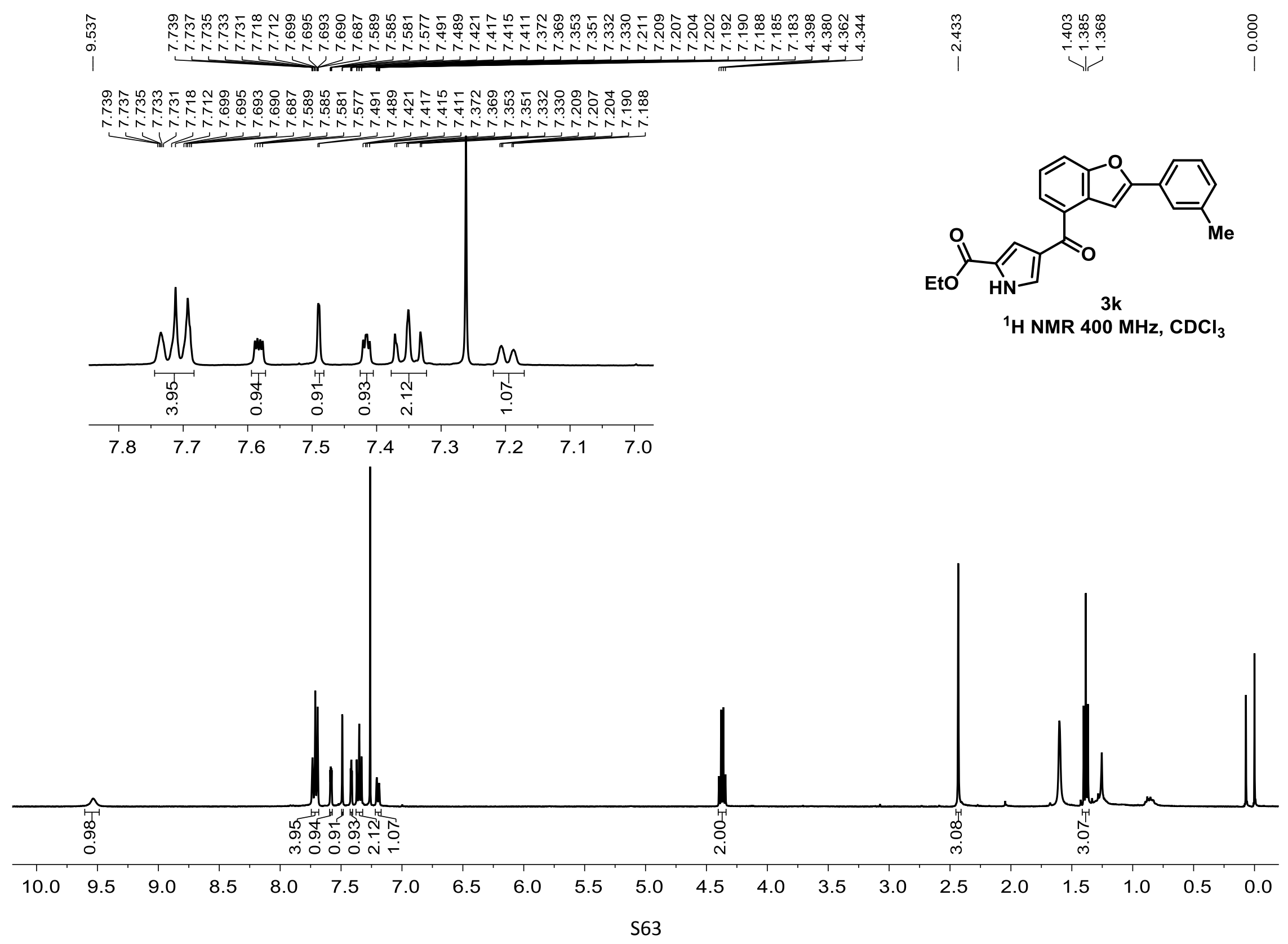




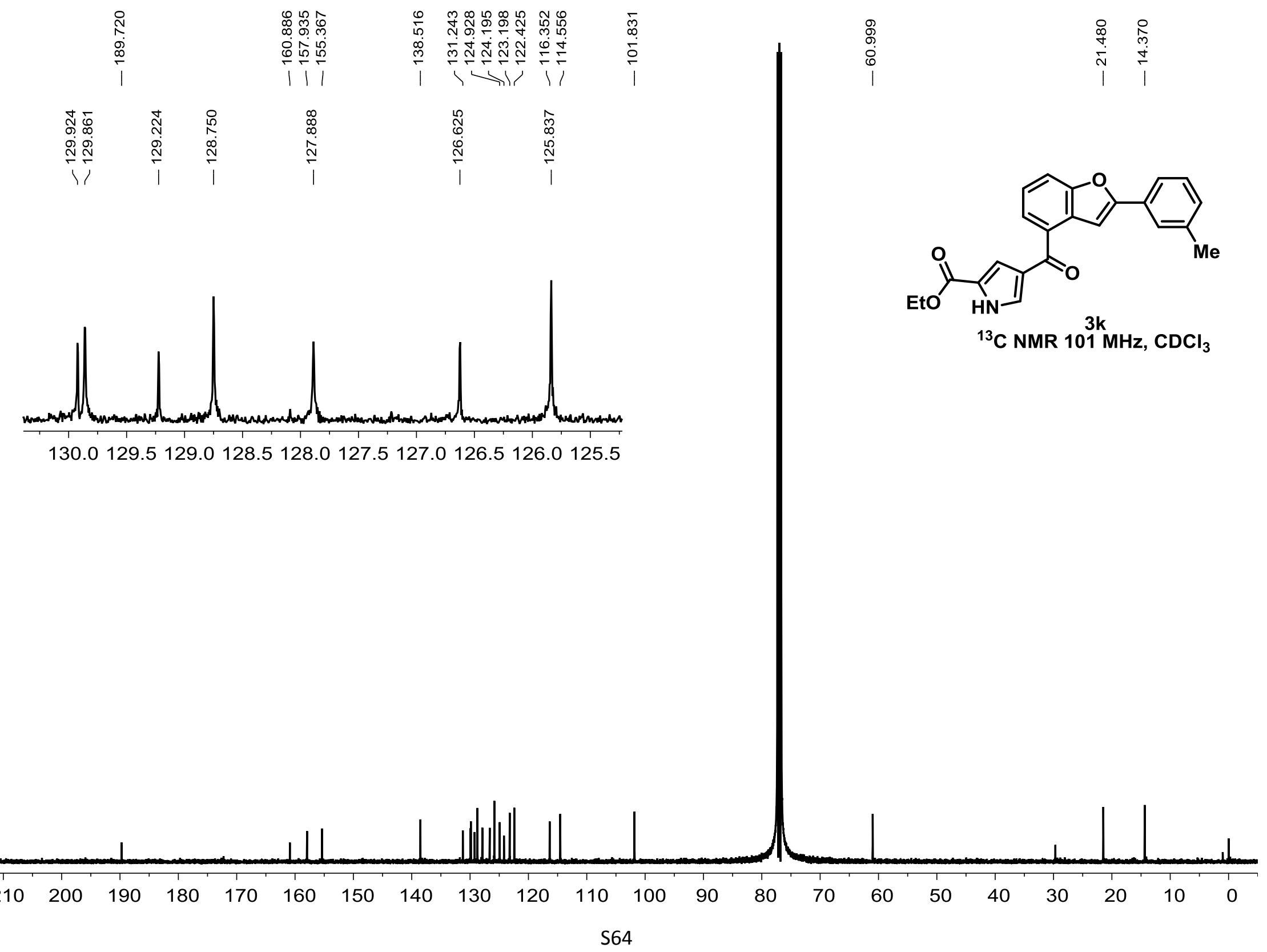




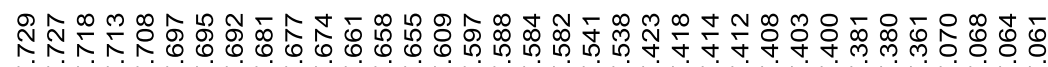

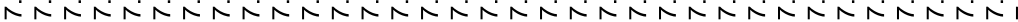
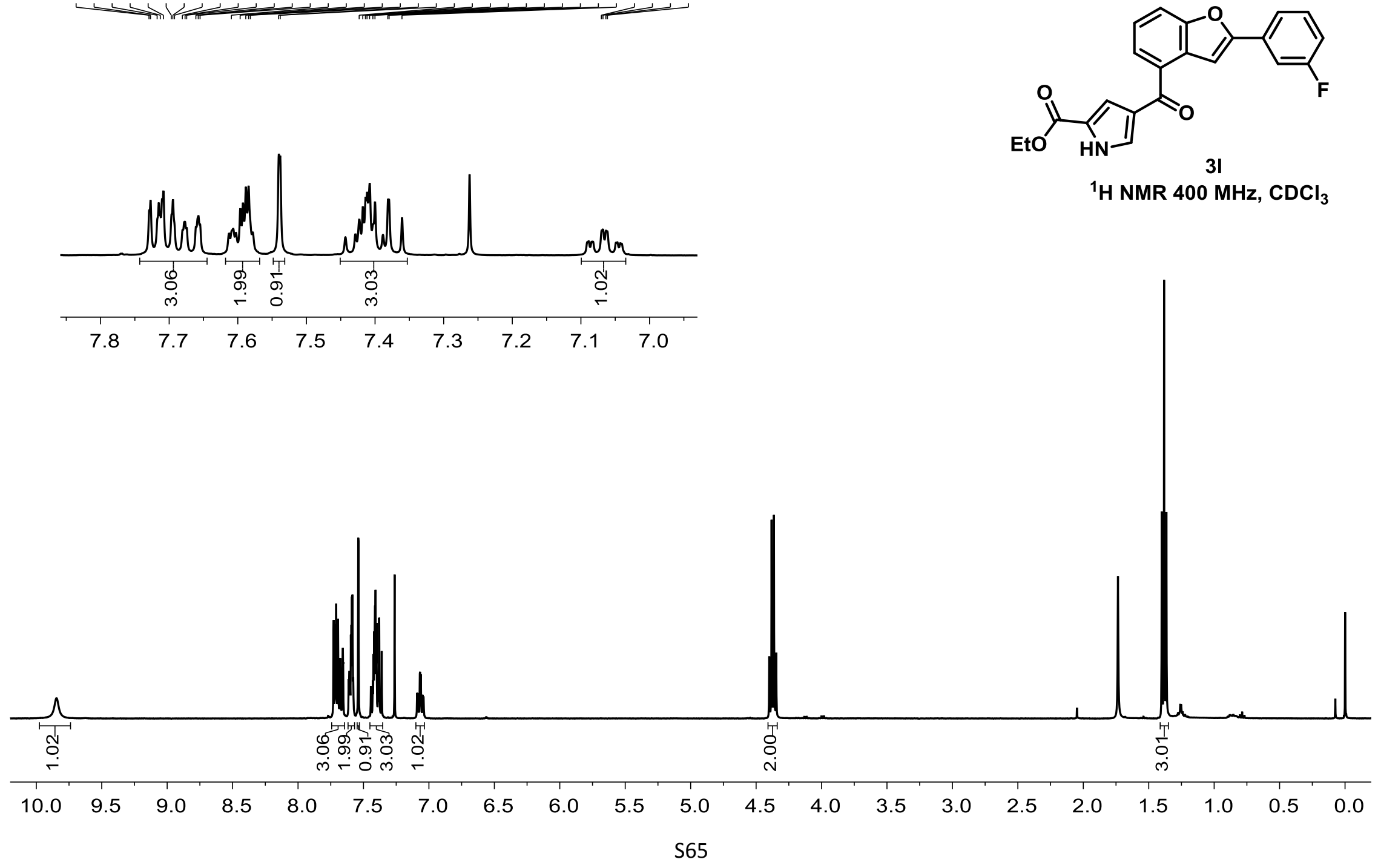


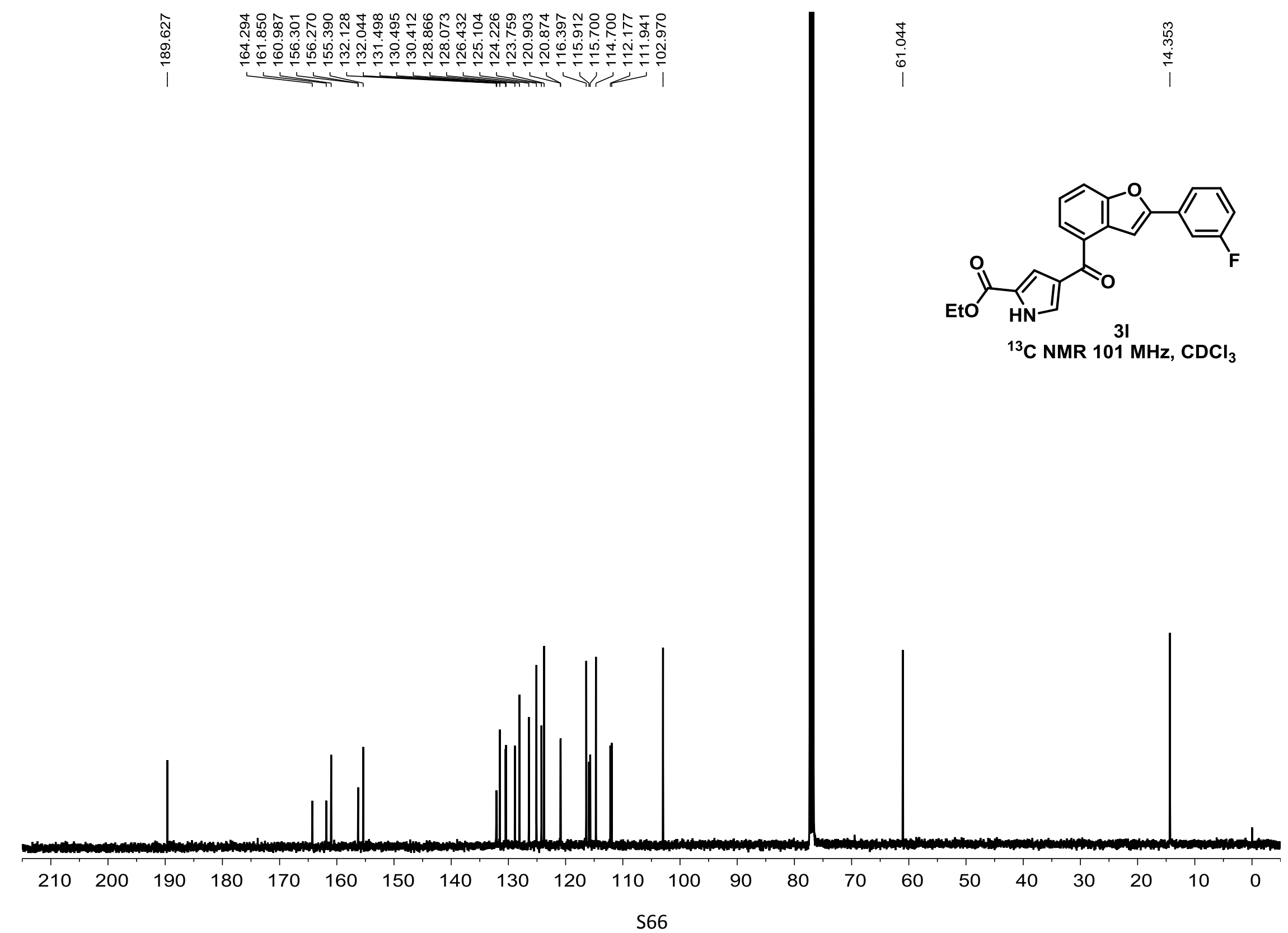




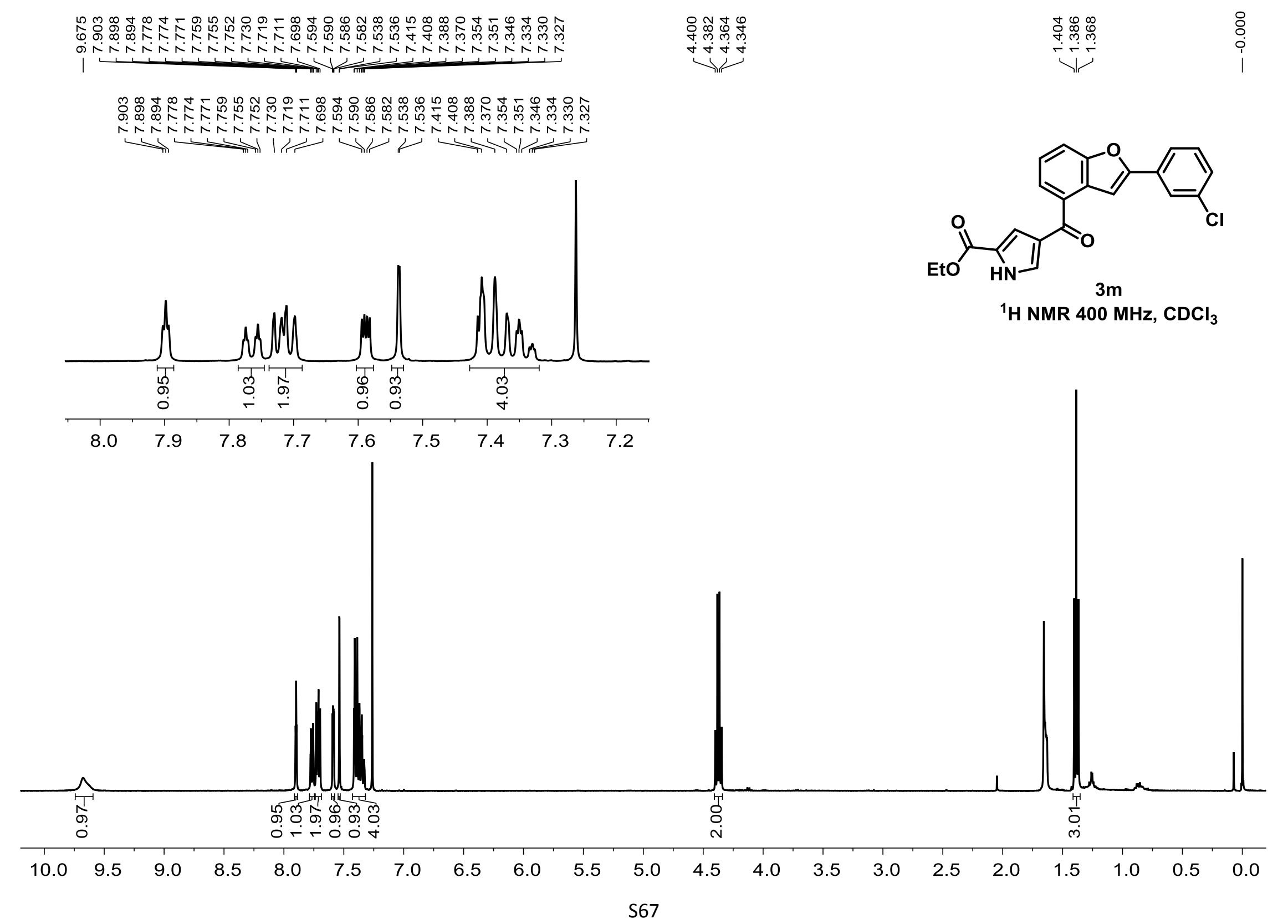



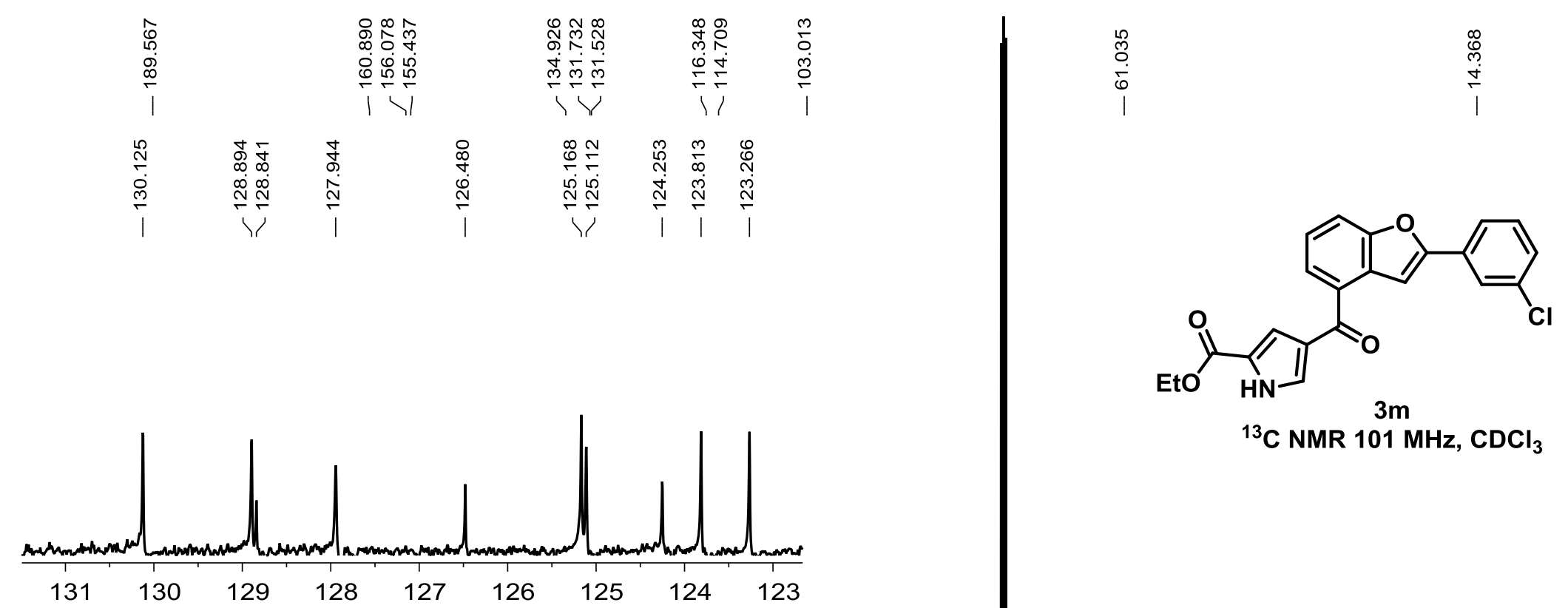

${ }^{3} \mathrm{C}$ NMR $101 \mathrm{MHz}, \mathrm{CDCl}_{3}$

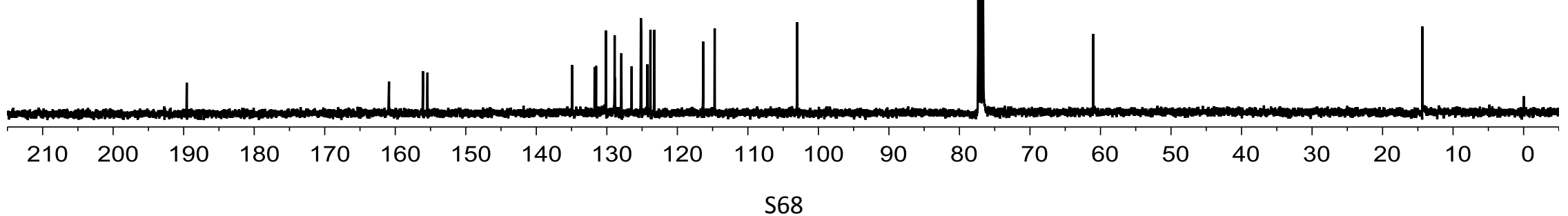




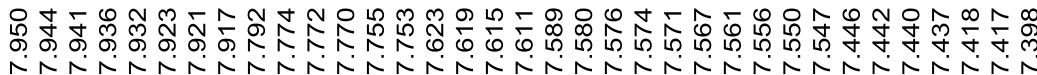
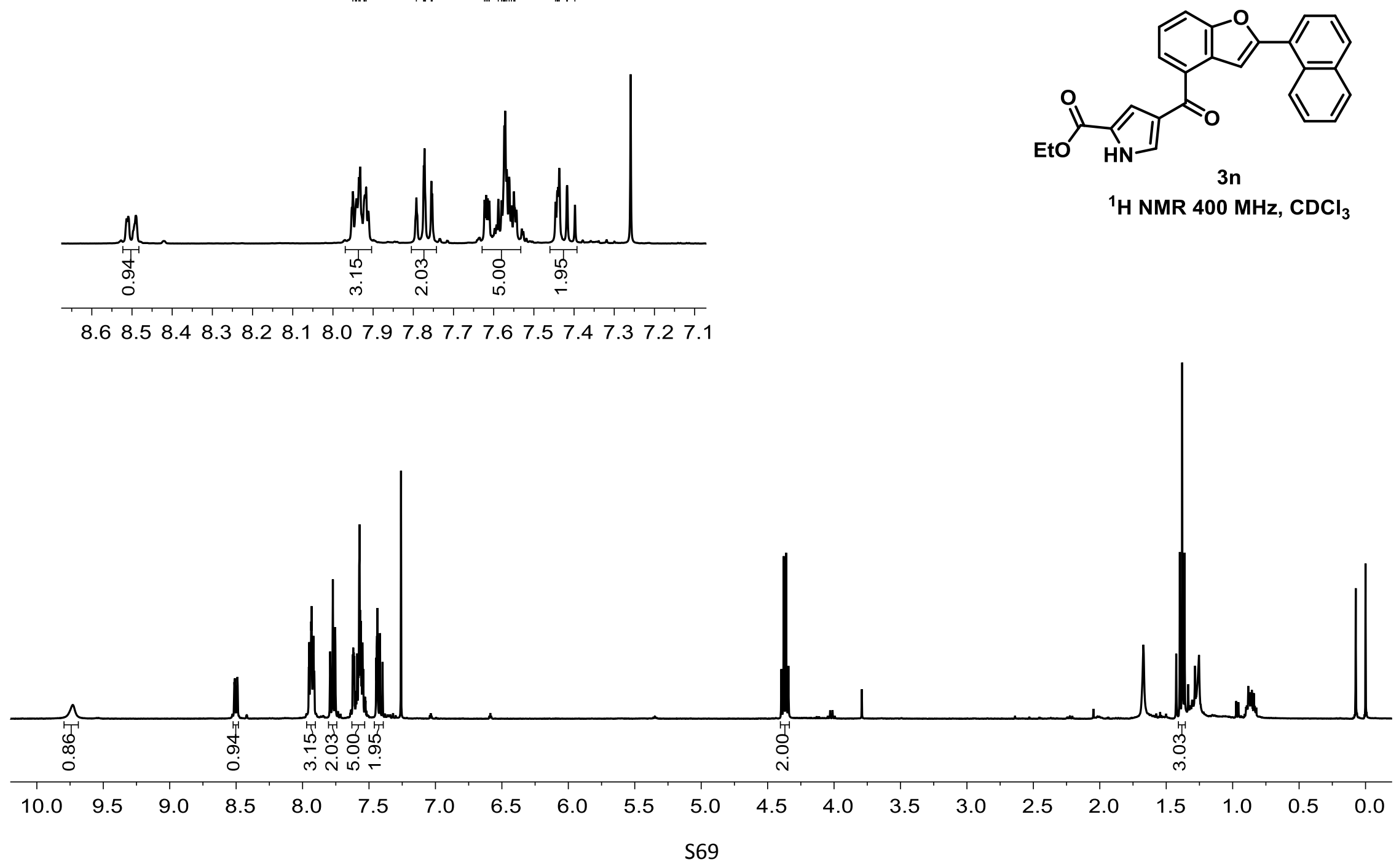


$$
\text { i l }
$$

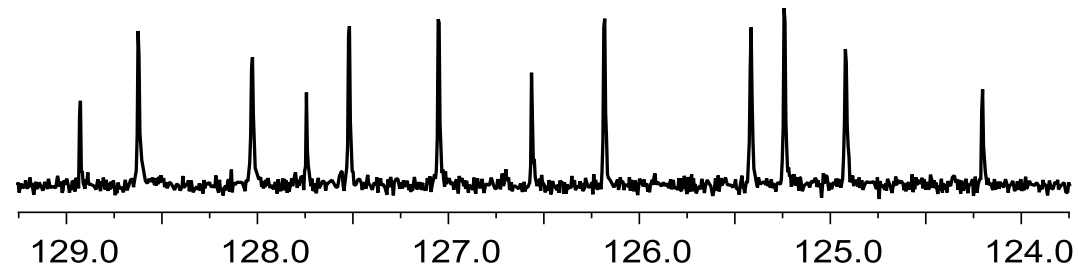

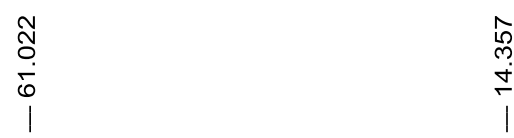
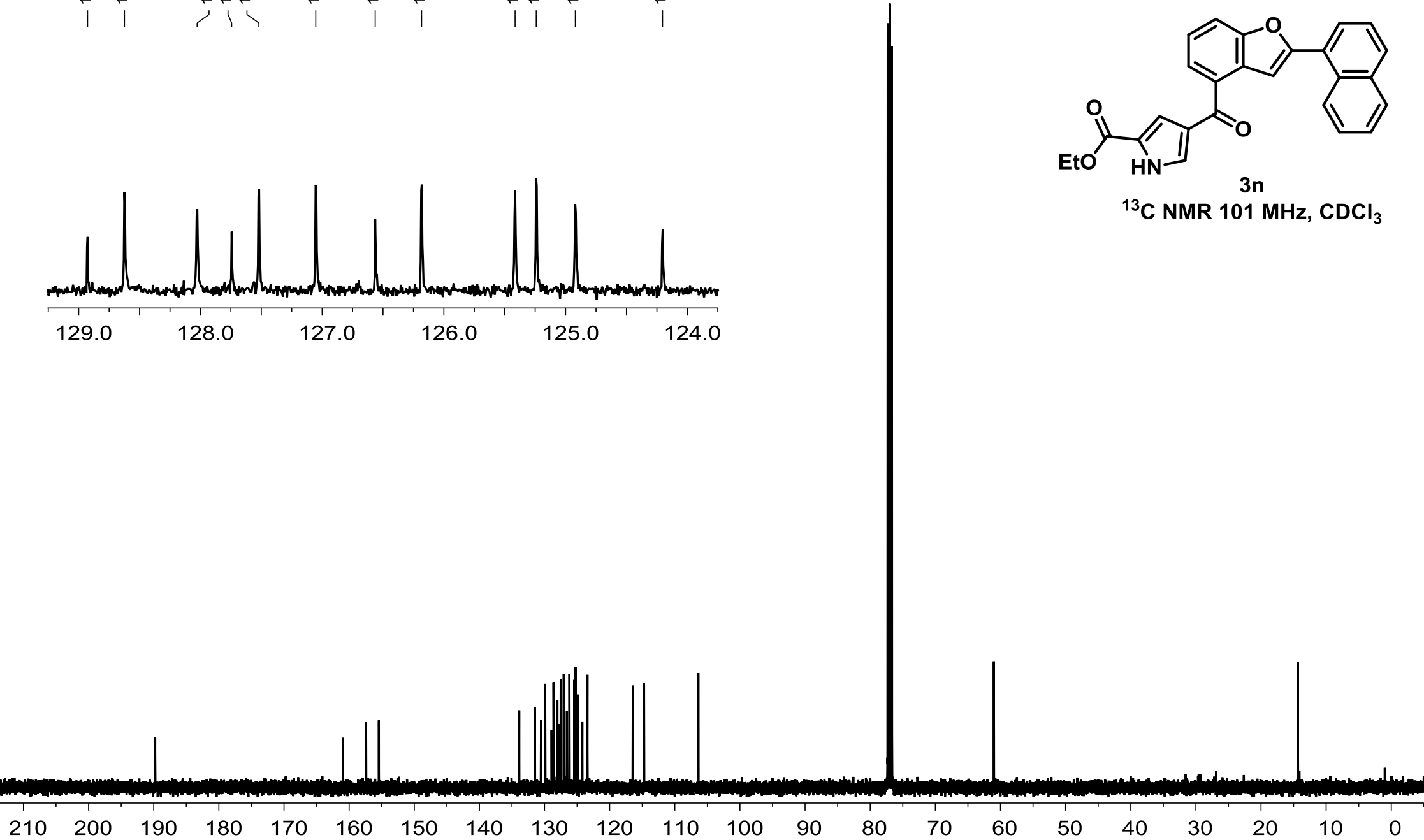


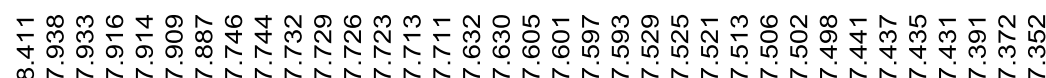

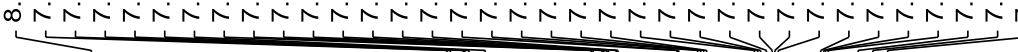
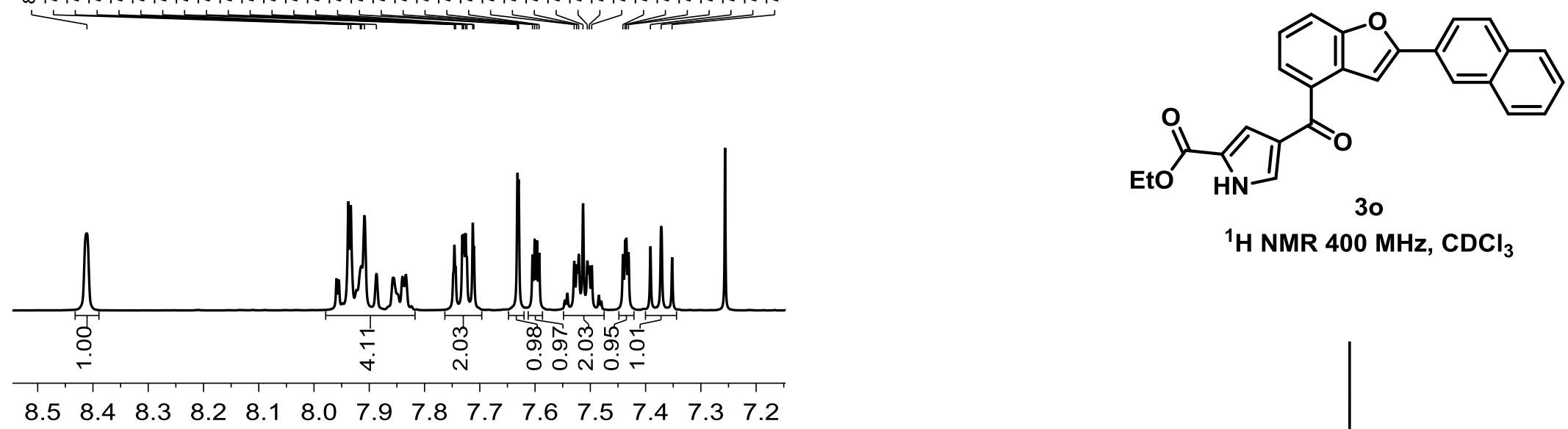

$\begin{array}{lllllllllllllllll}8.5 & 8.4 & 8.3 & 8.2 & 8.1 & 8.0 & 7.9 & 7.8 & 7.7 & 7.6 & 7.5 & 7.4 & 7.3 & 7.2\end{array}$

$\mathrm{H}$ NMR $400 \mathrm{MHz}, \mathrm{CDCl}_{3}$

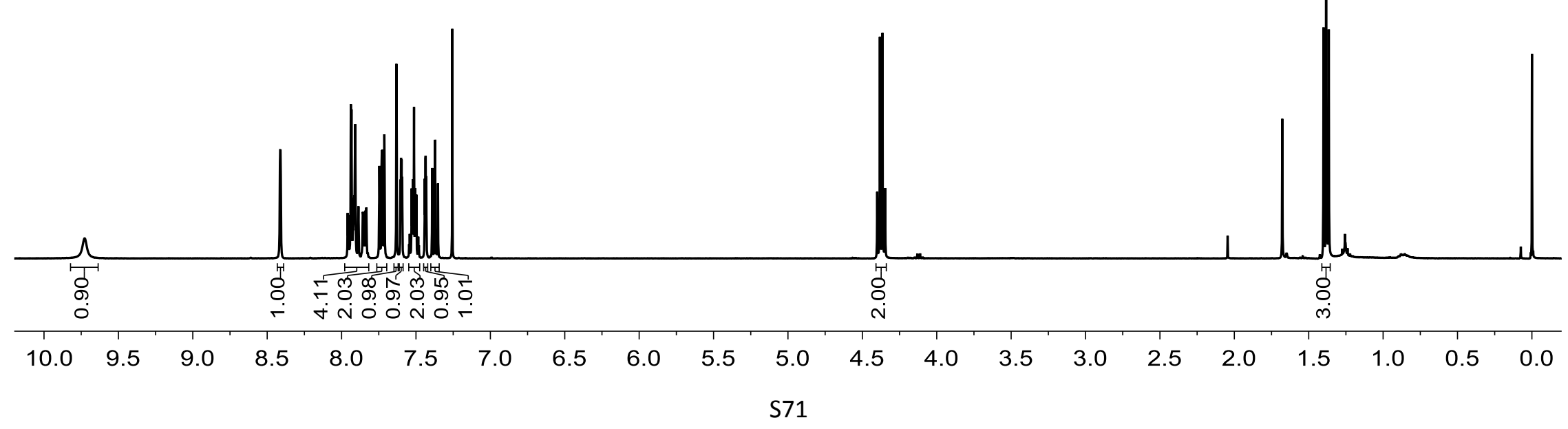




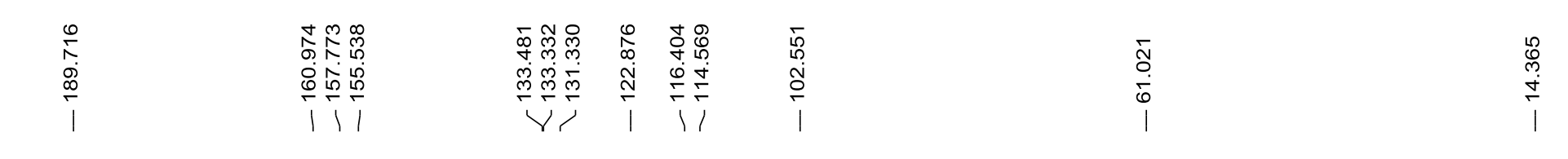

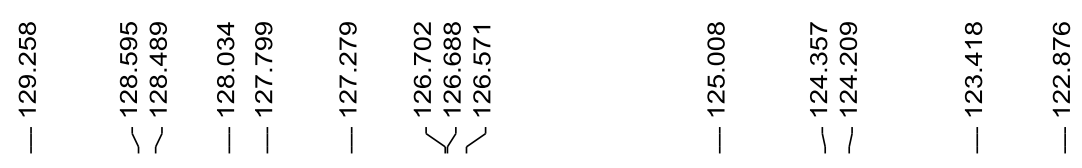
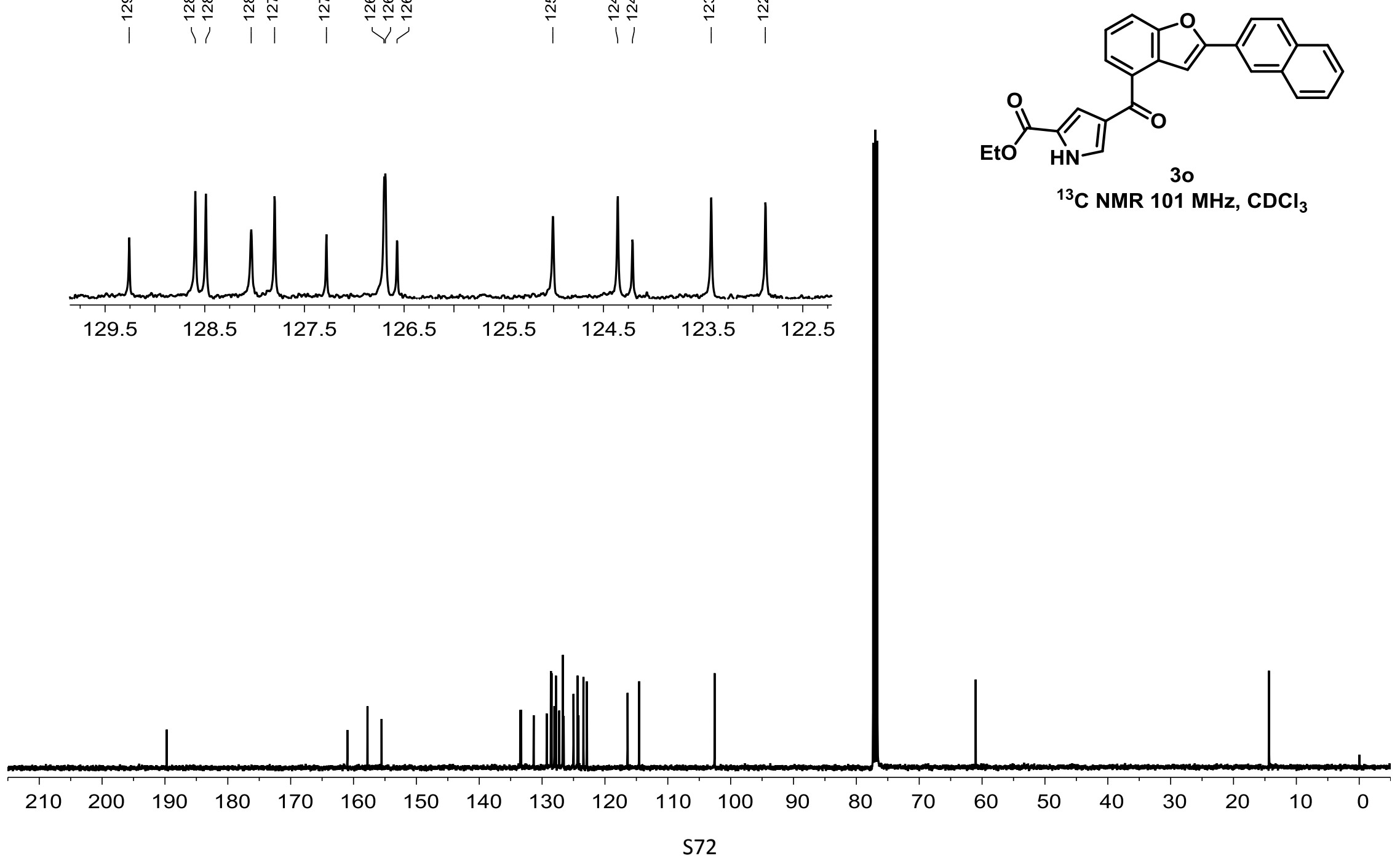


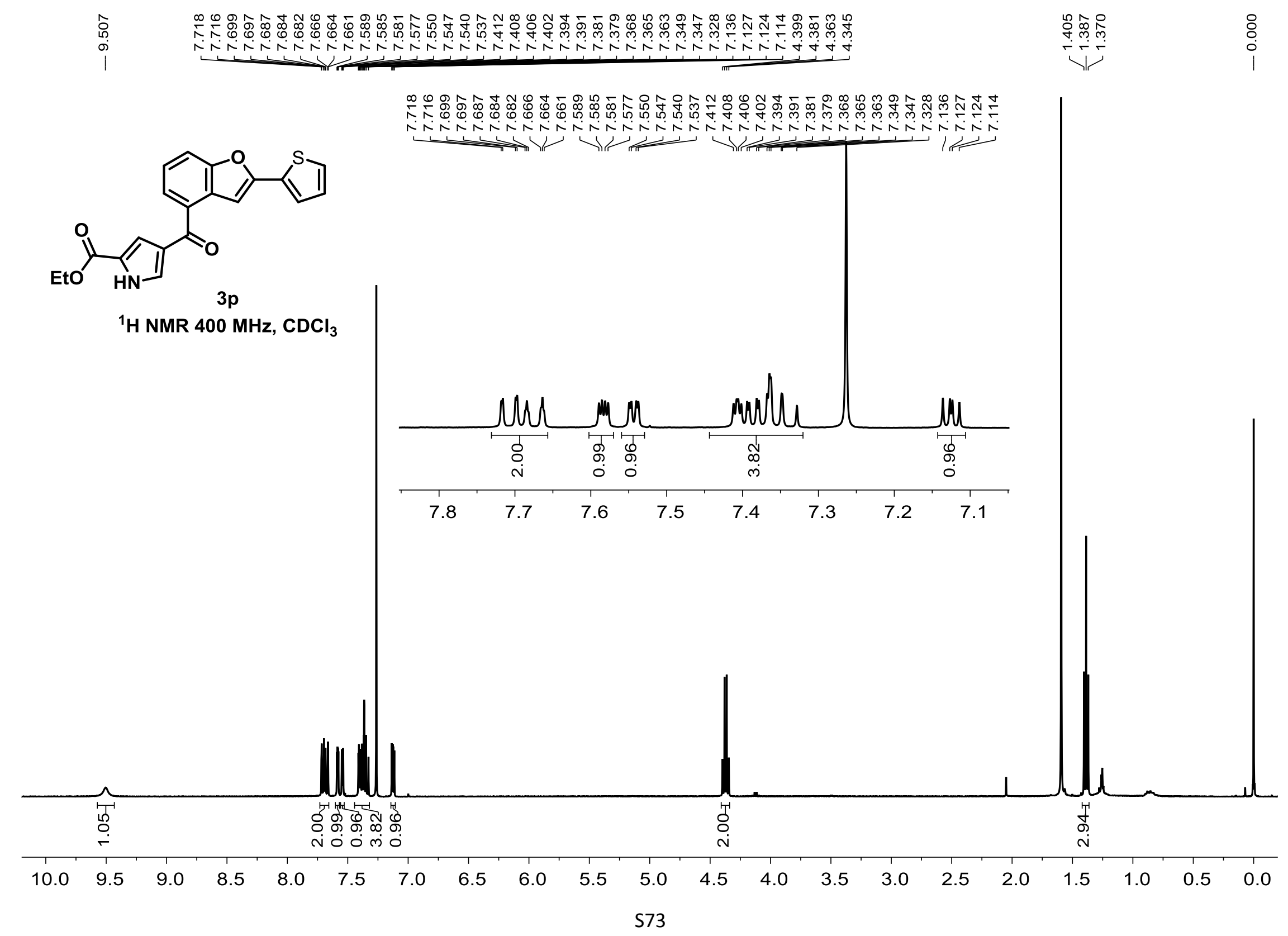




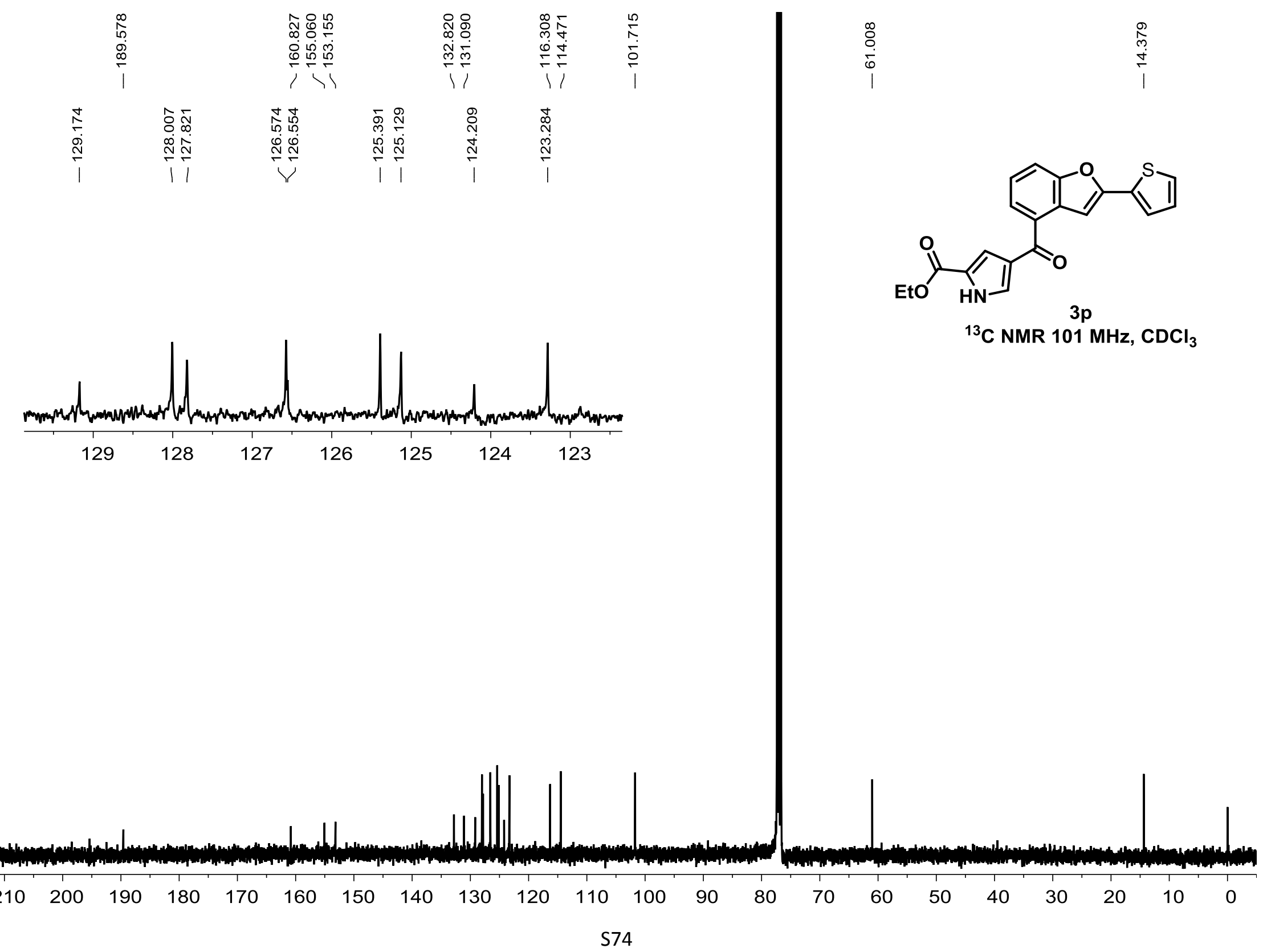




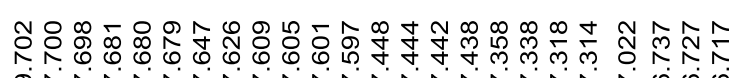

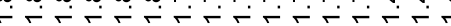
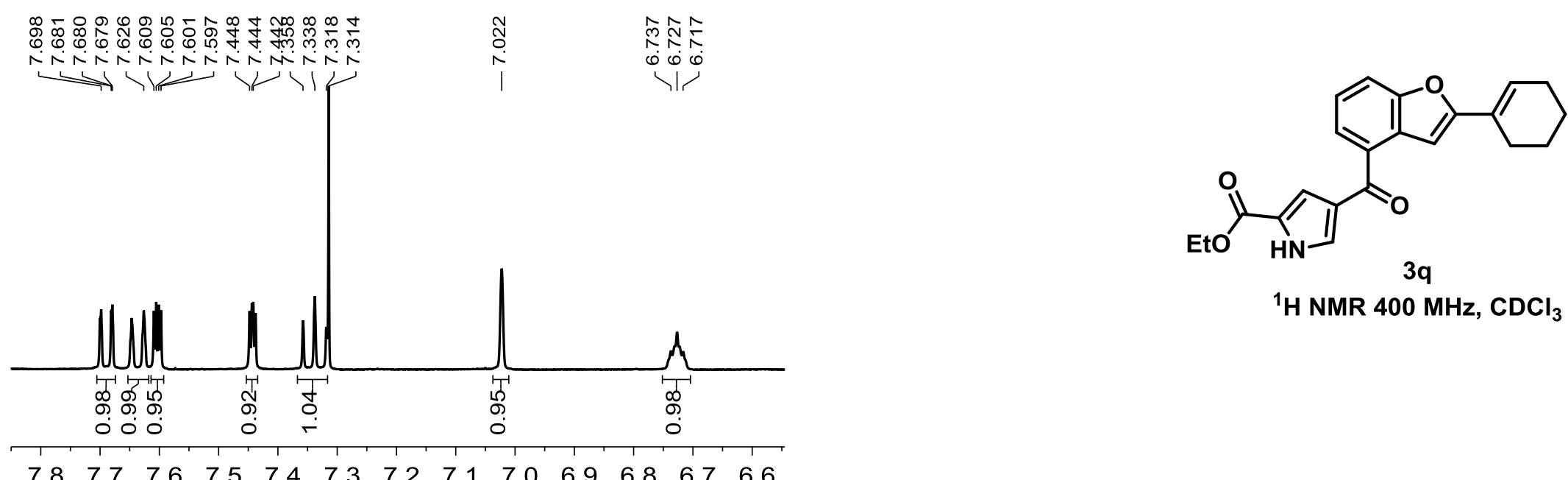

${ }^{1} \mathrm{H}$ NMR $400 \mathrm{MHz}, \mathrm{CDCl}_{3}$

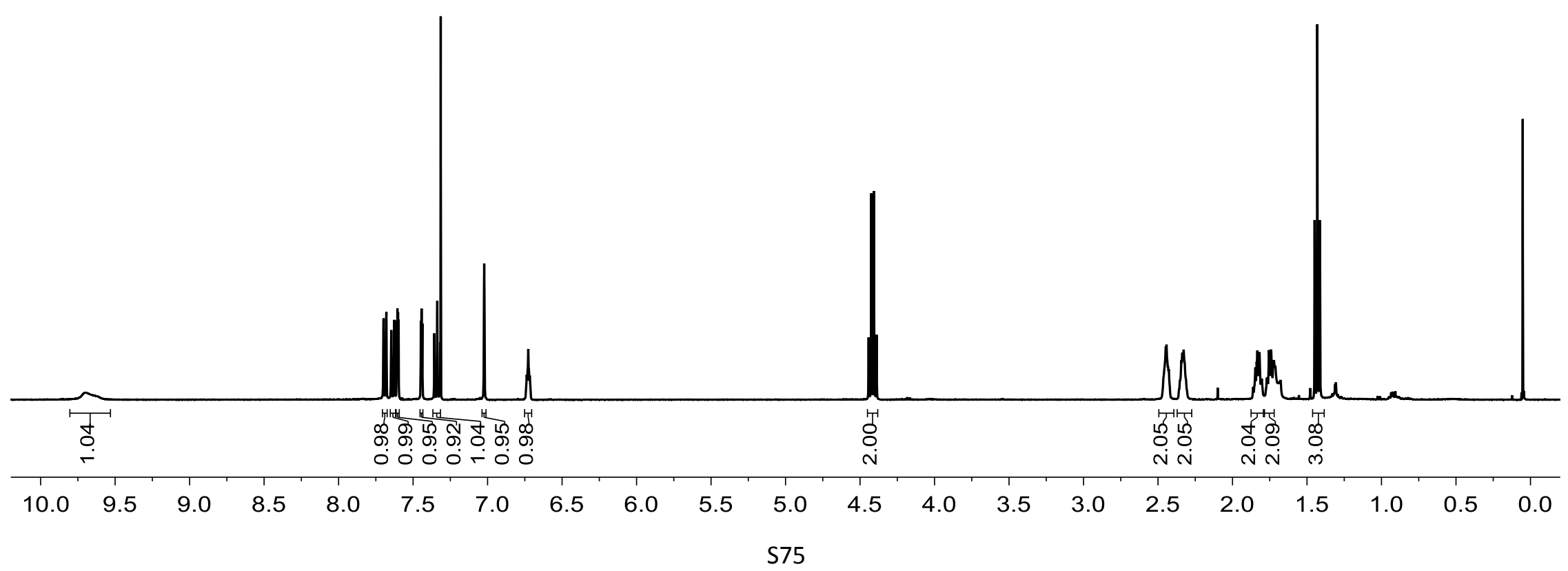




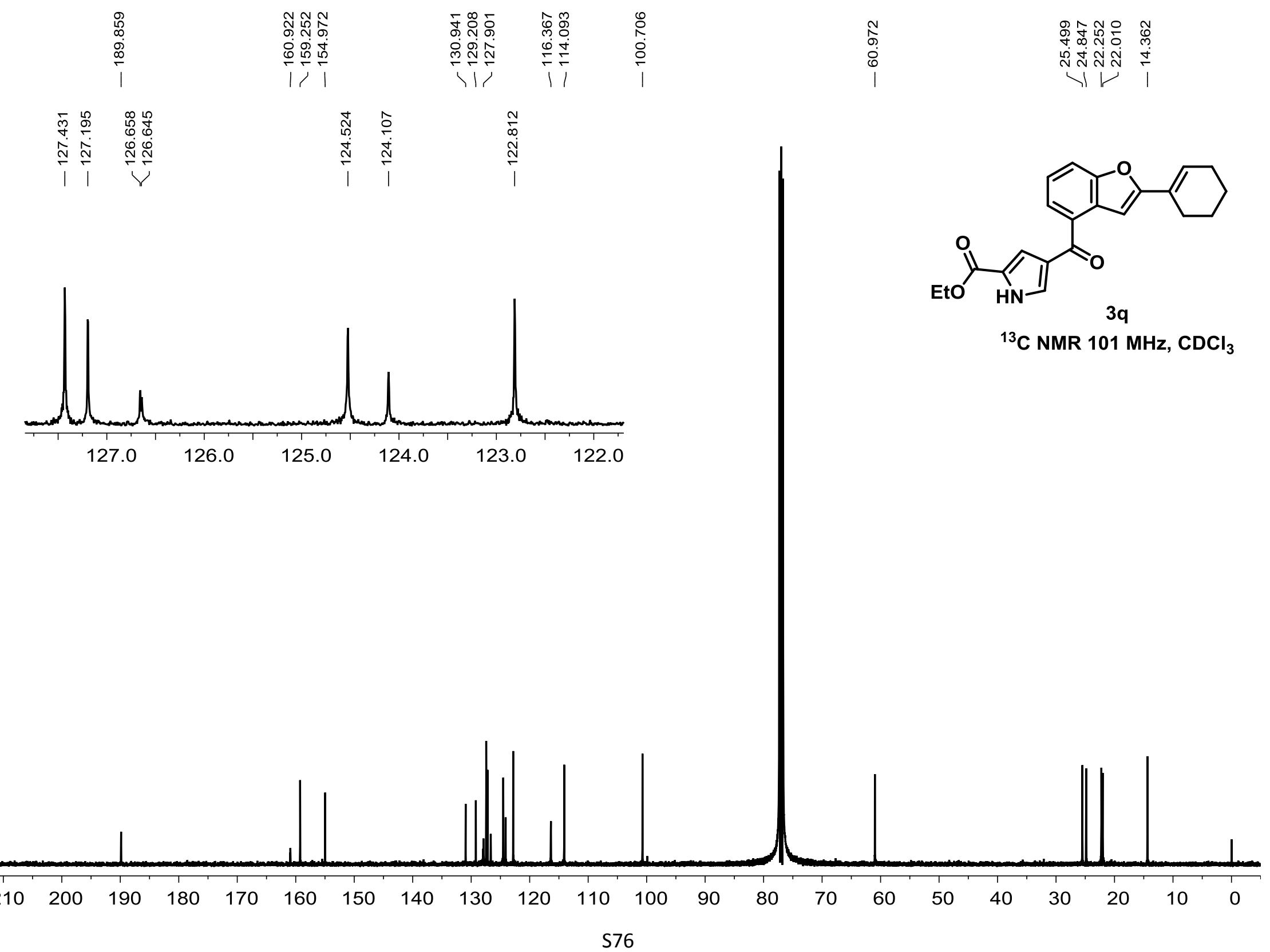




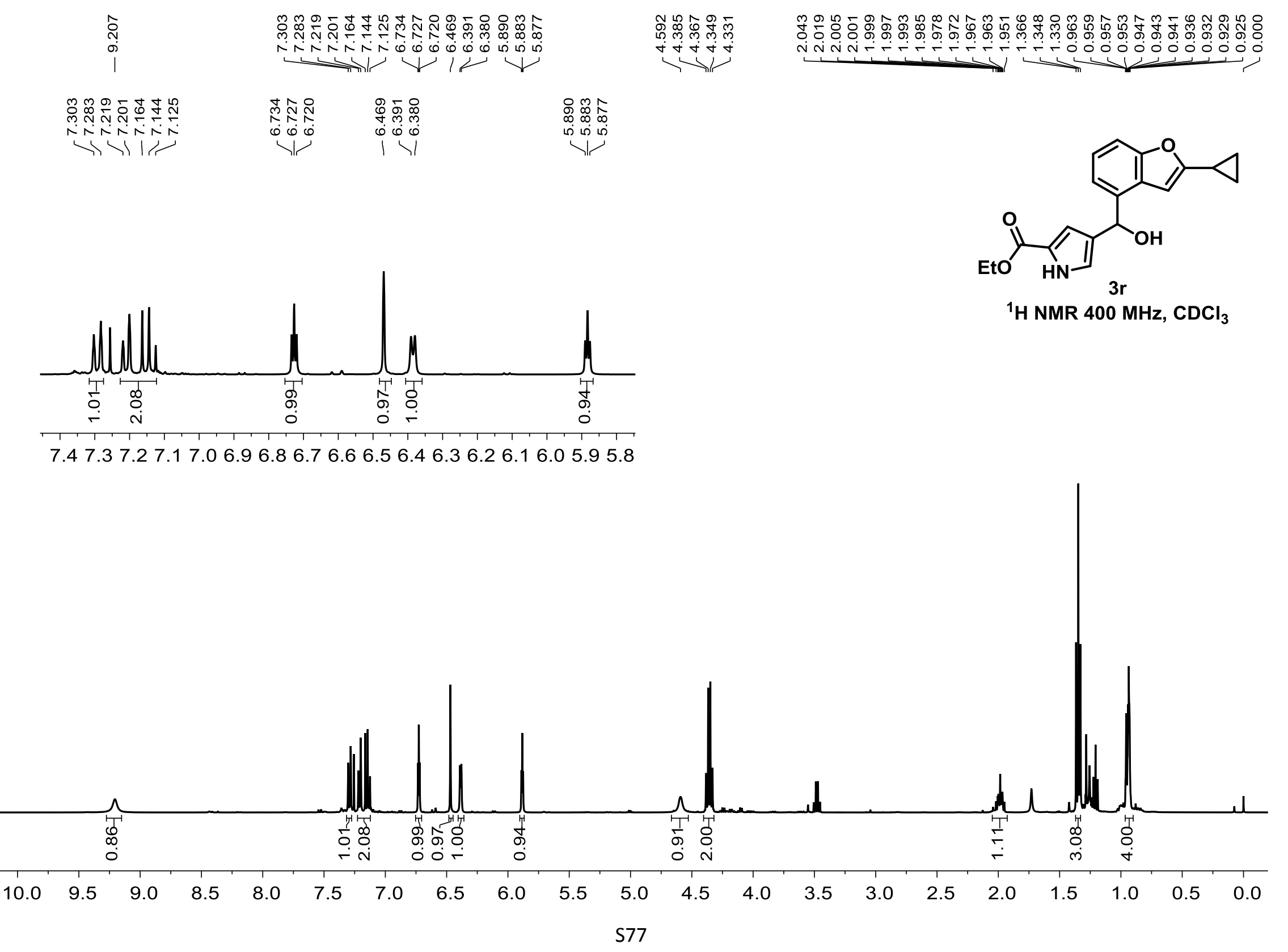




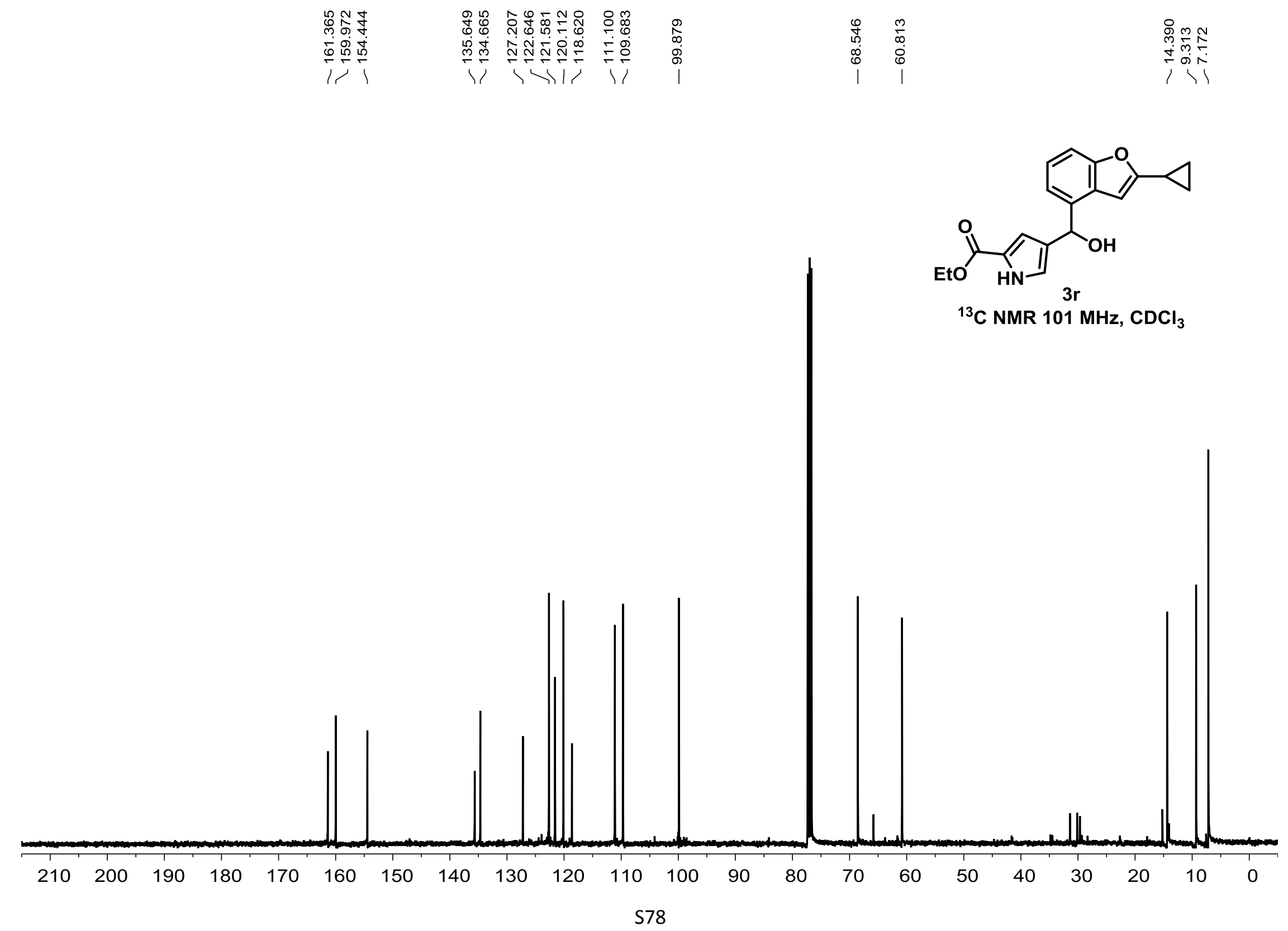




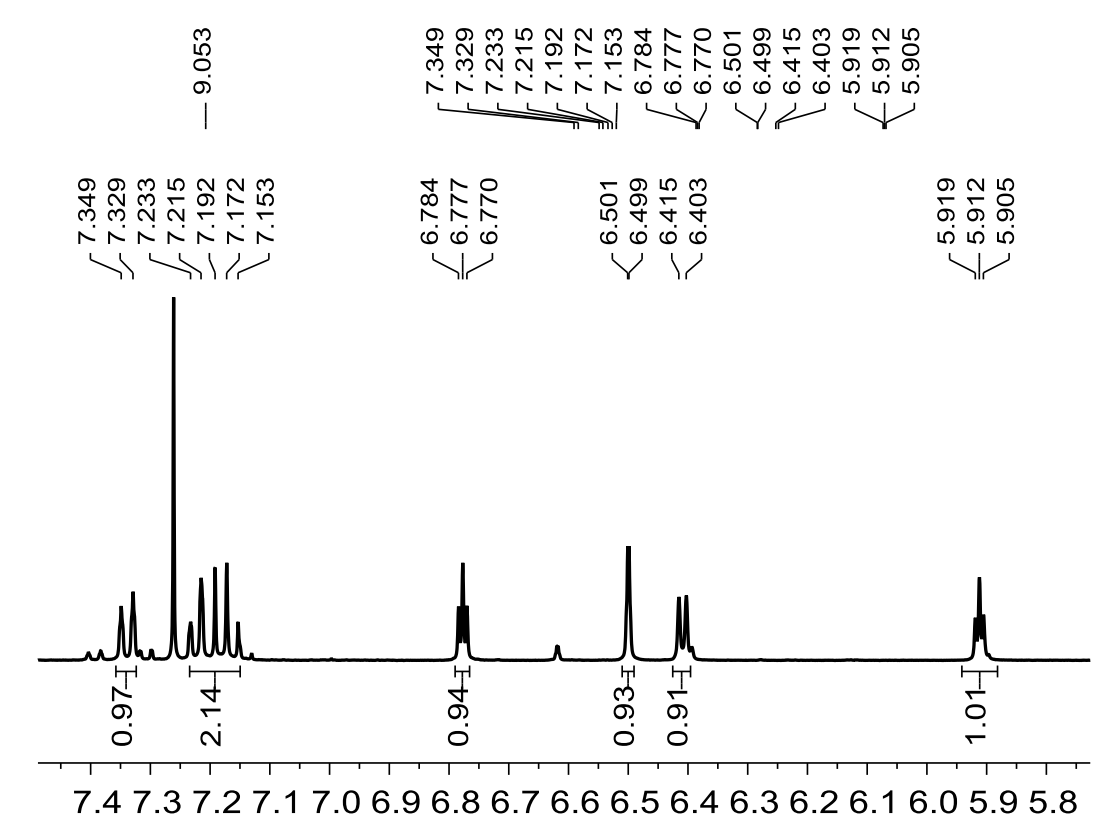

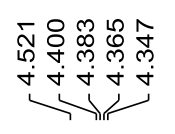

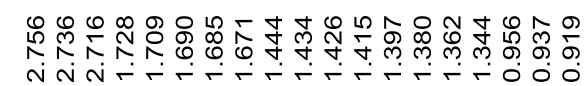

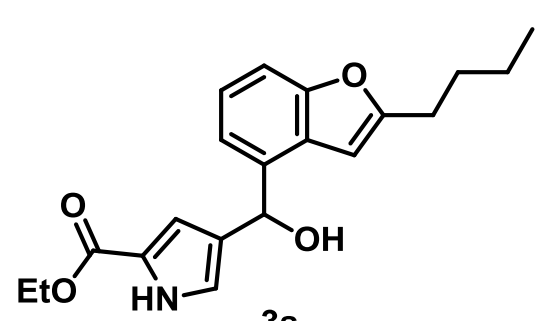

${ }^{1} \mathrm{H}$ NMR $400 \mathrm{MHz} \mathrm{CDCl}_{3}$

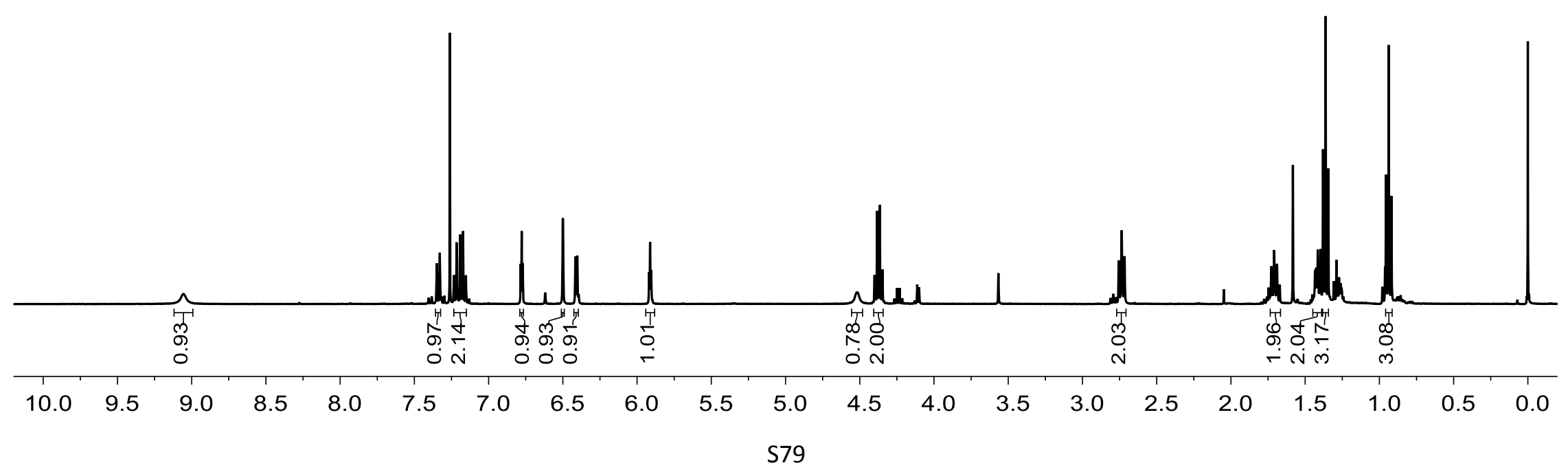




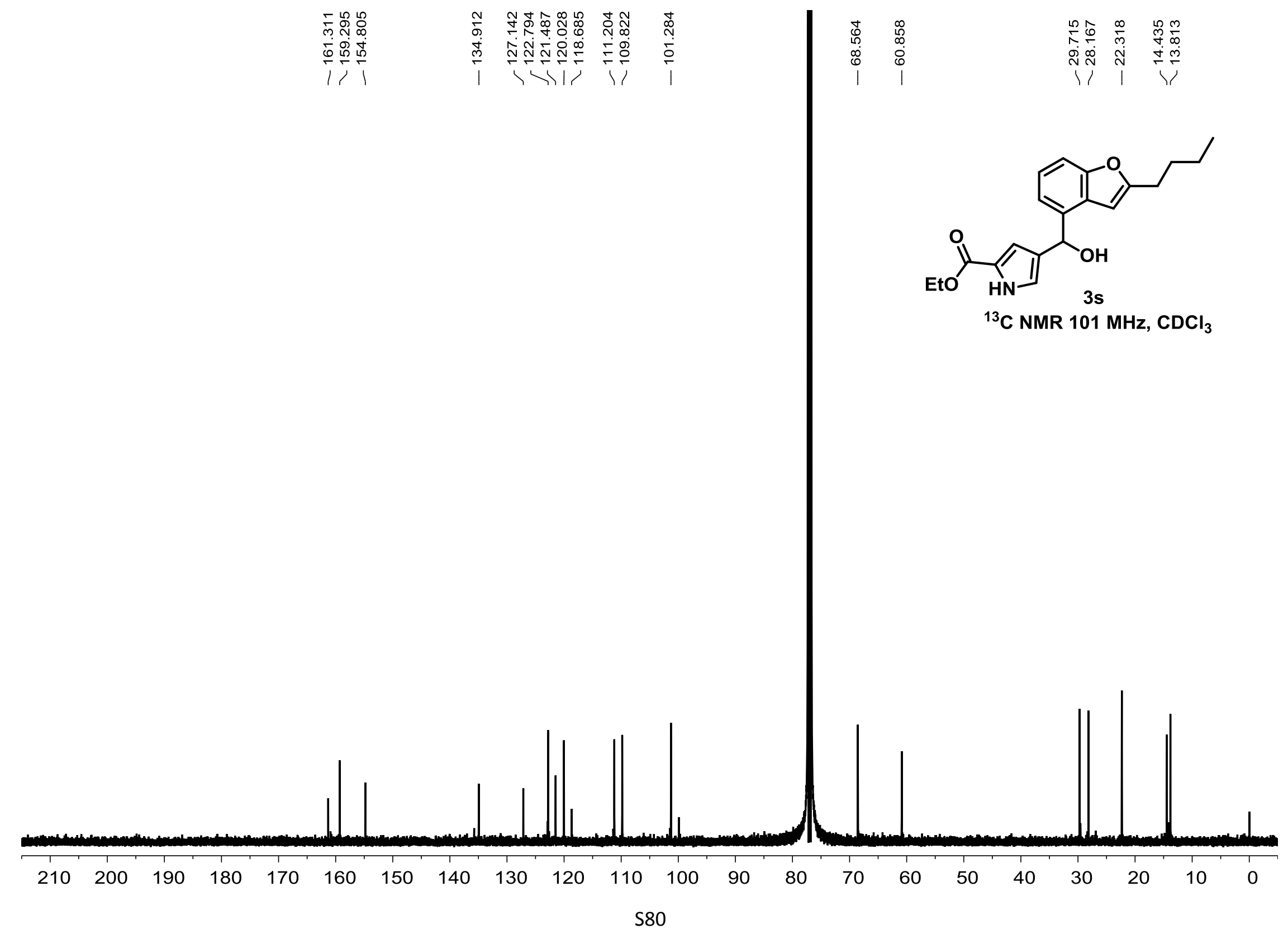




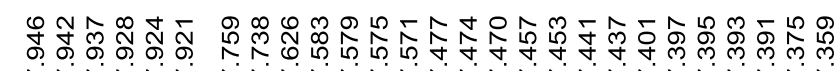

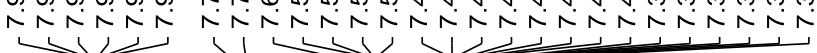

$\begin{array}{ccc}9 & \infty \\ 0 & 0 \\ 0 & 0 \\ 0 & 0 \\ 0 & 0 \\ 1 & 0\end{array}$
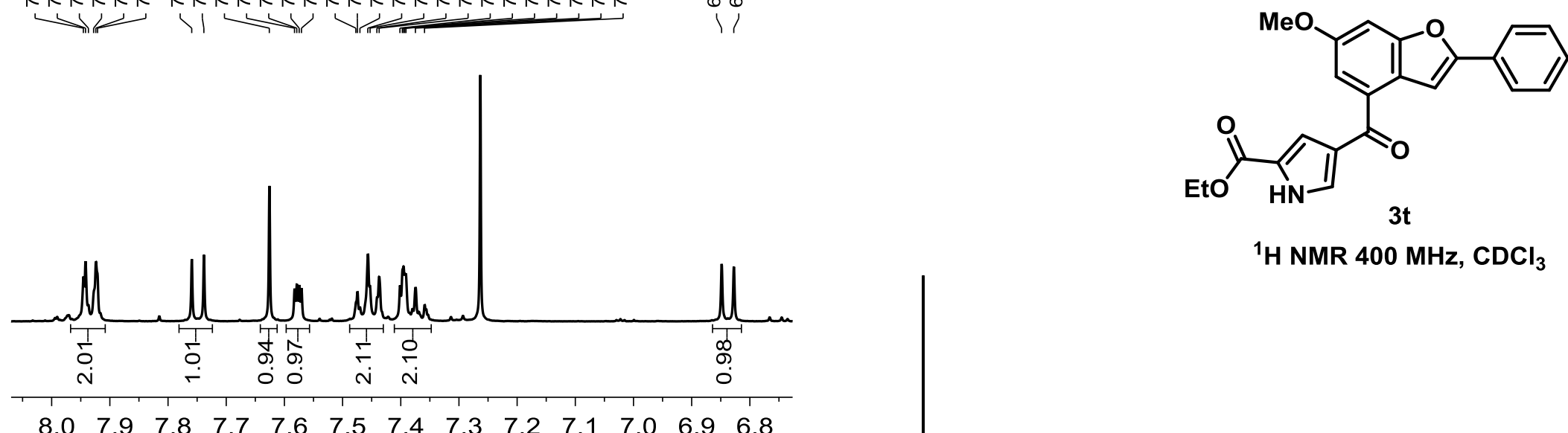

${ }^{1} \mathrm{H}$ NMR $400 \mathrm{MHz}, \mathrm{CDCl}_{3}$
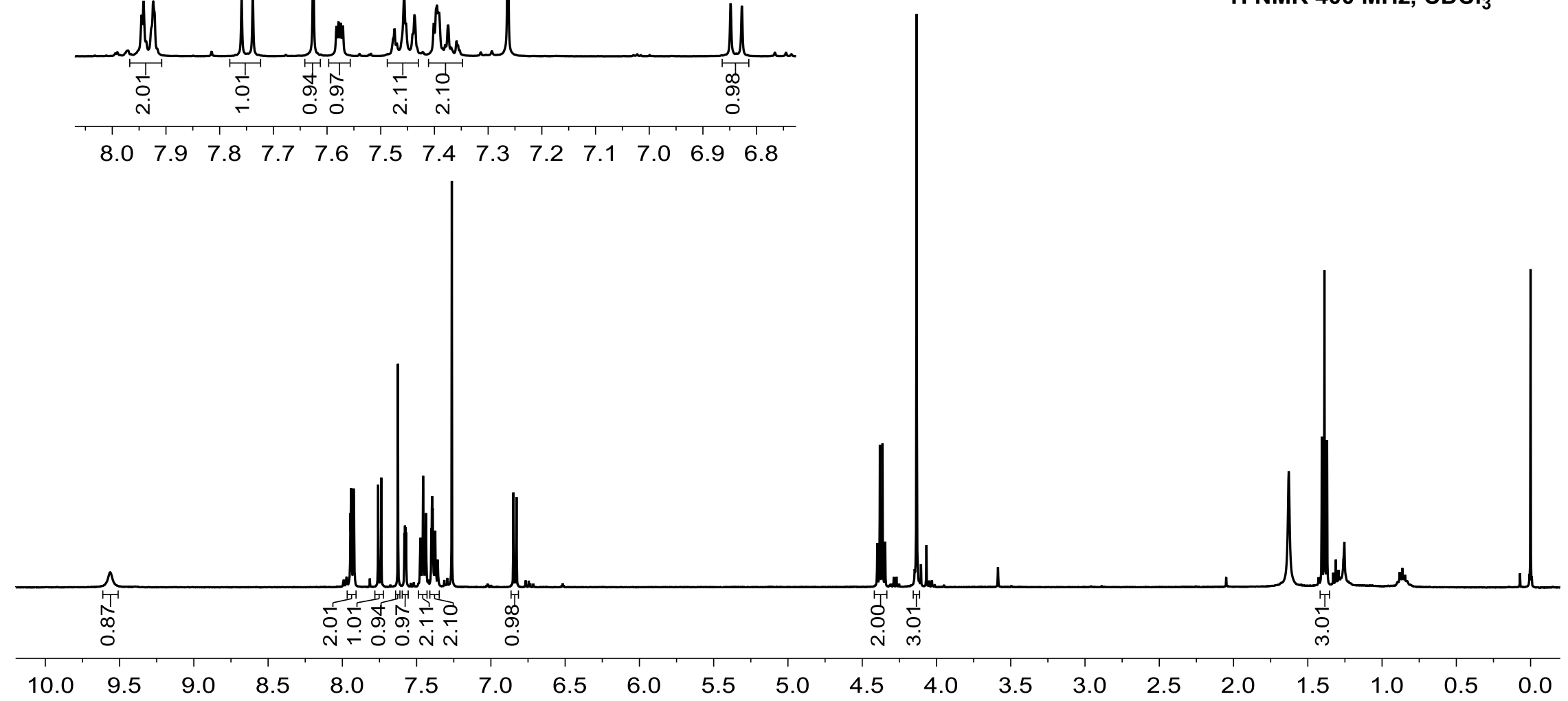


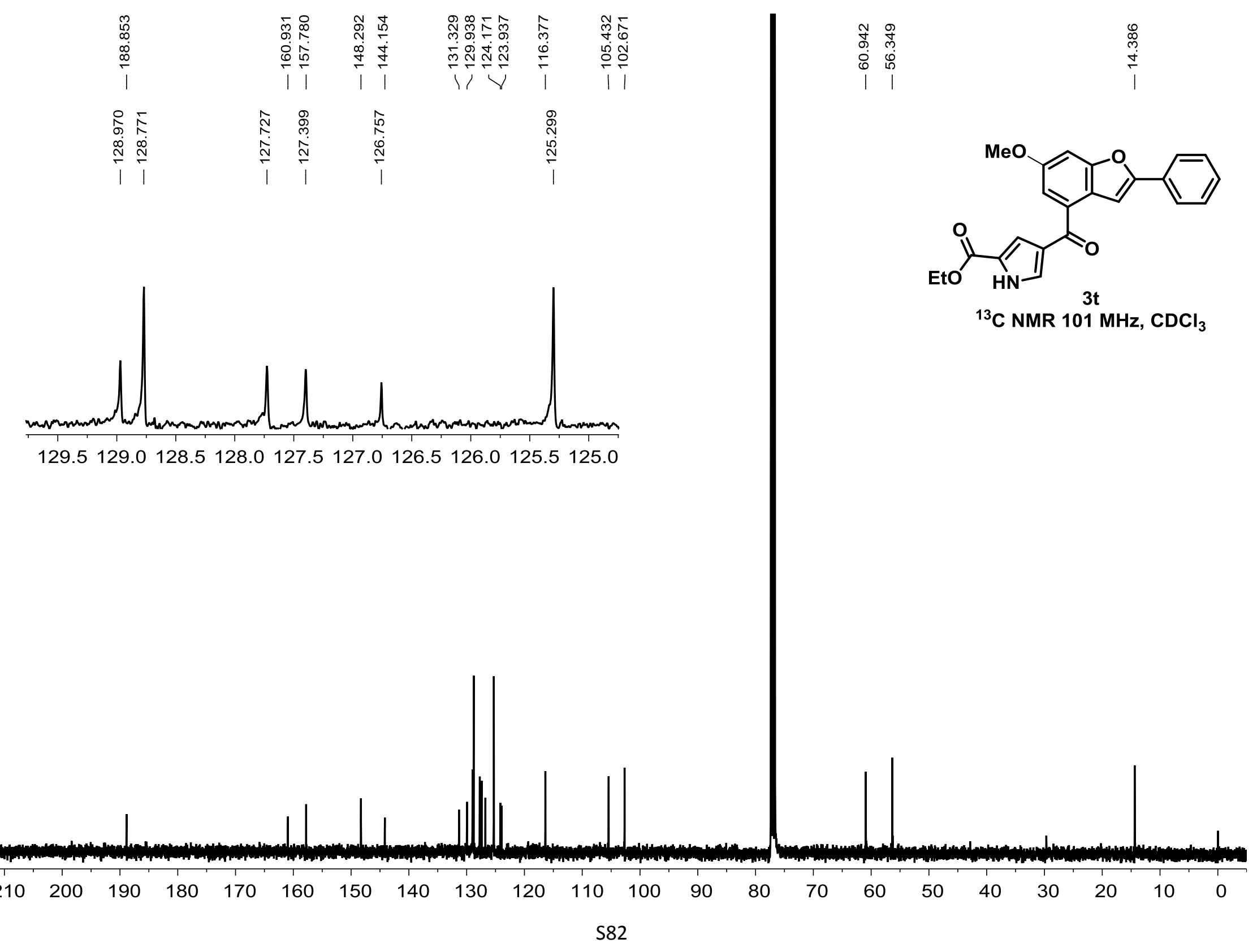




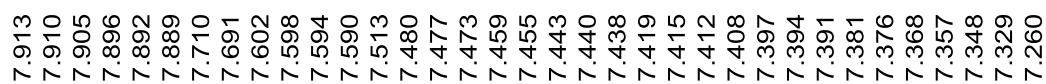

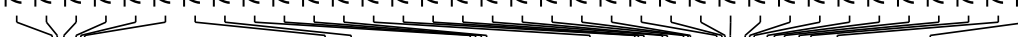

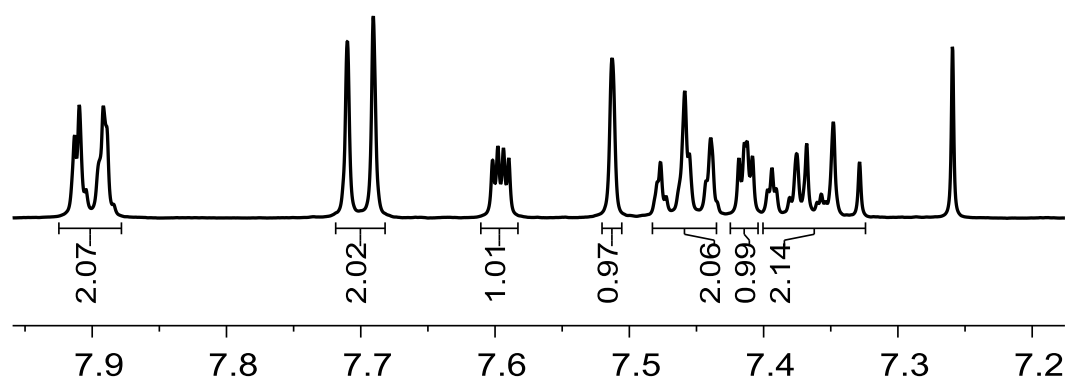

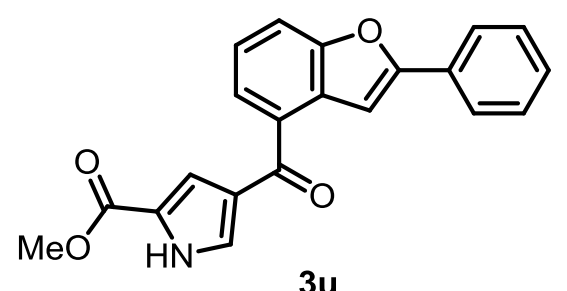

${ }^{1} \mathrm{H}$ NMR $400 \mathrm{MHz}^{\mathrm{CDCl}}{ }_{3}$

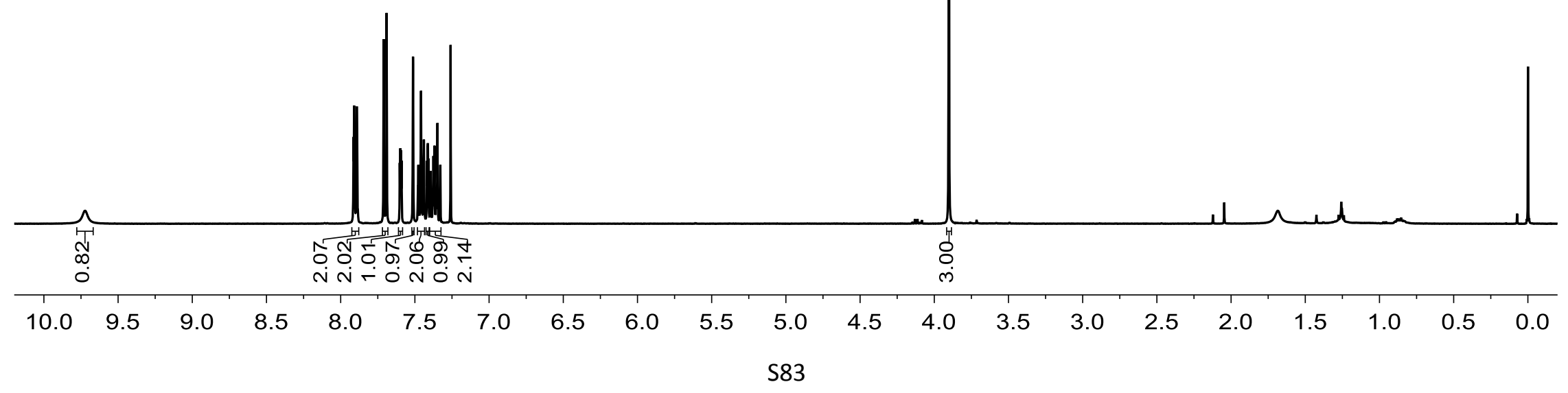




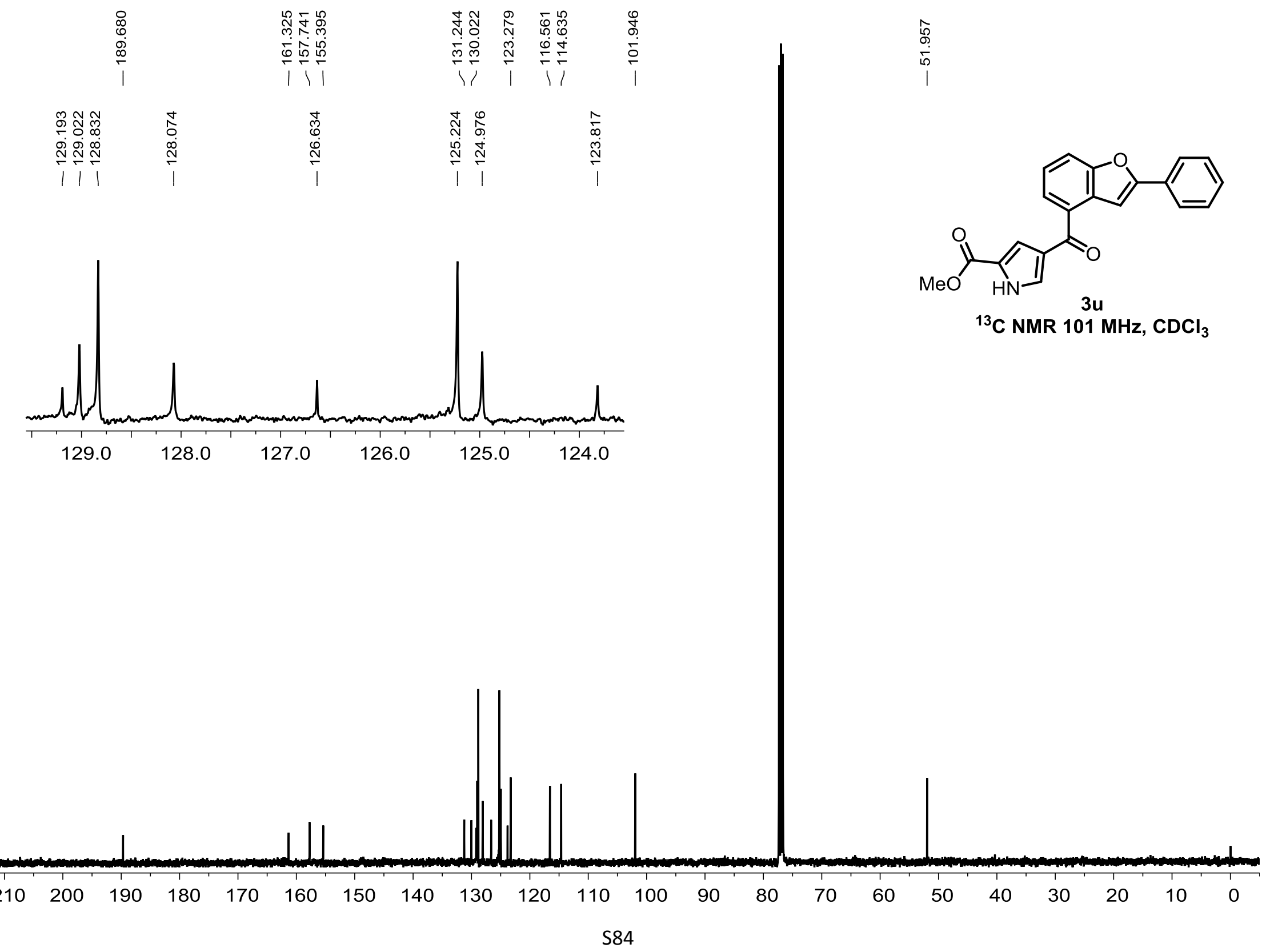




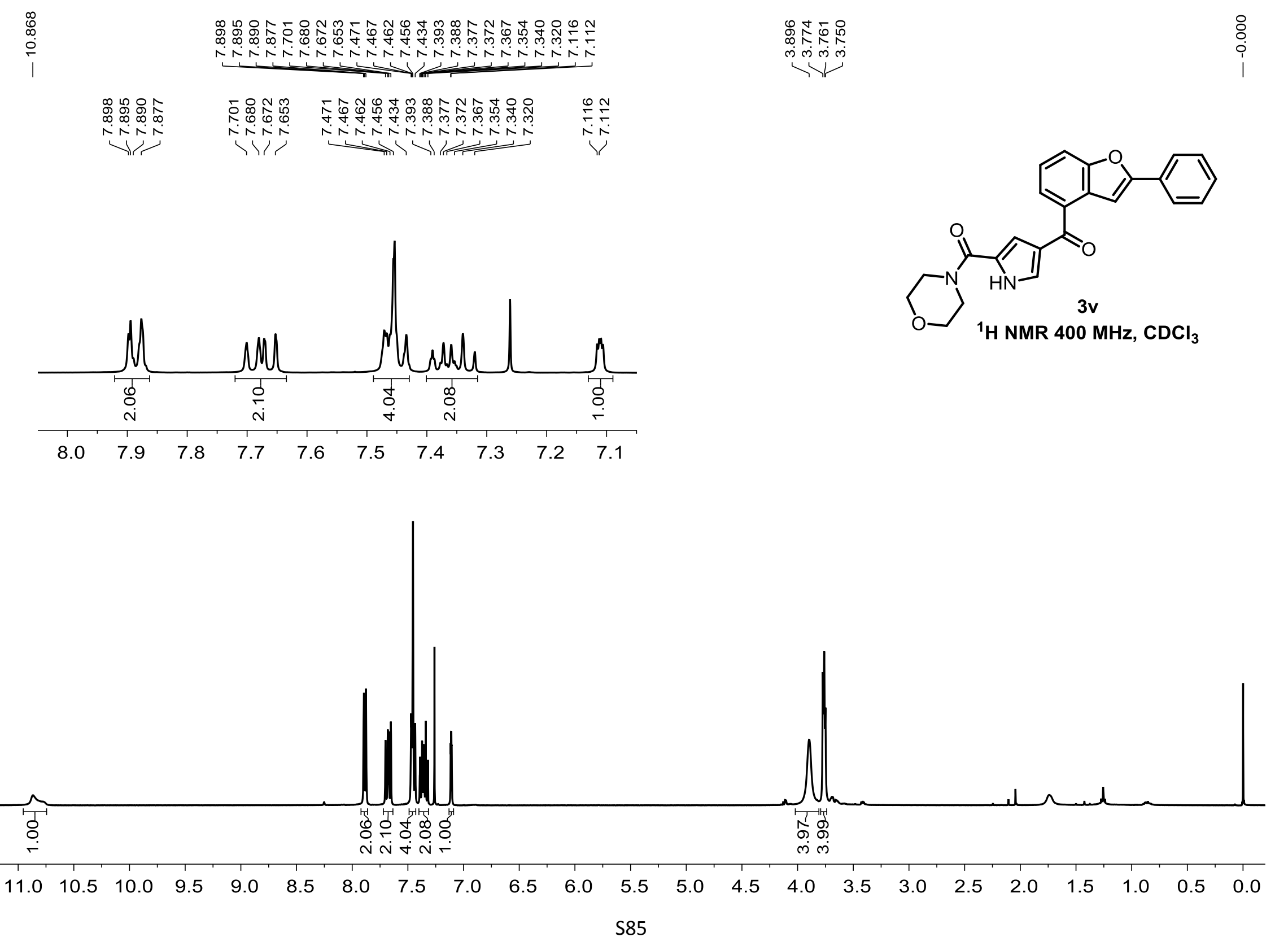




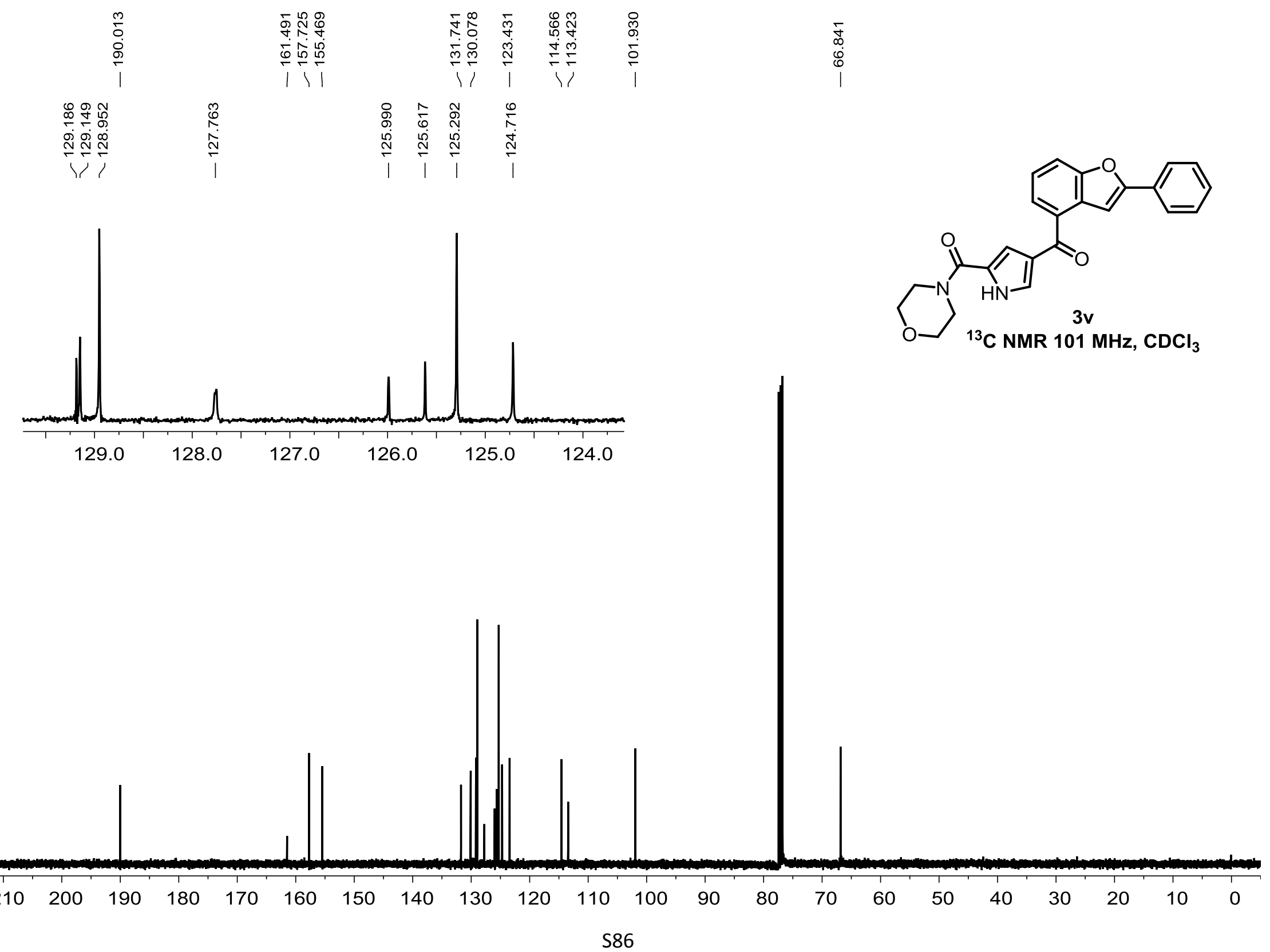




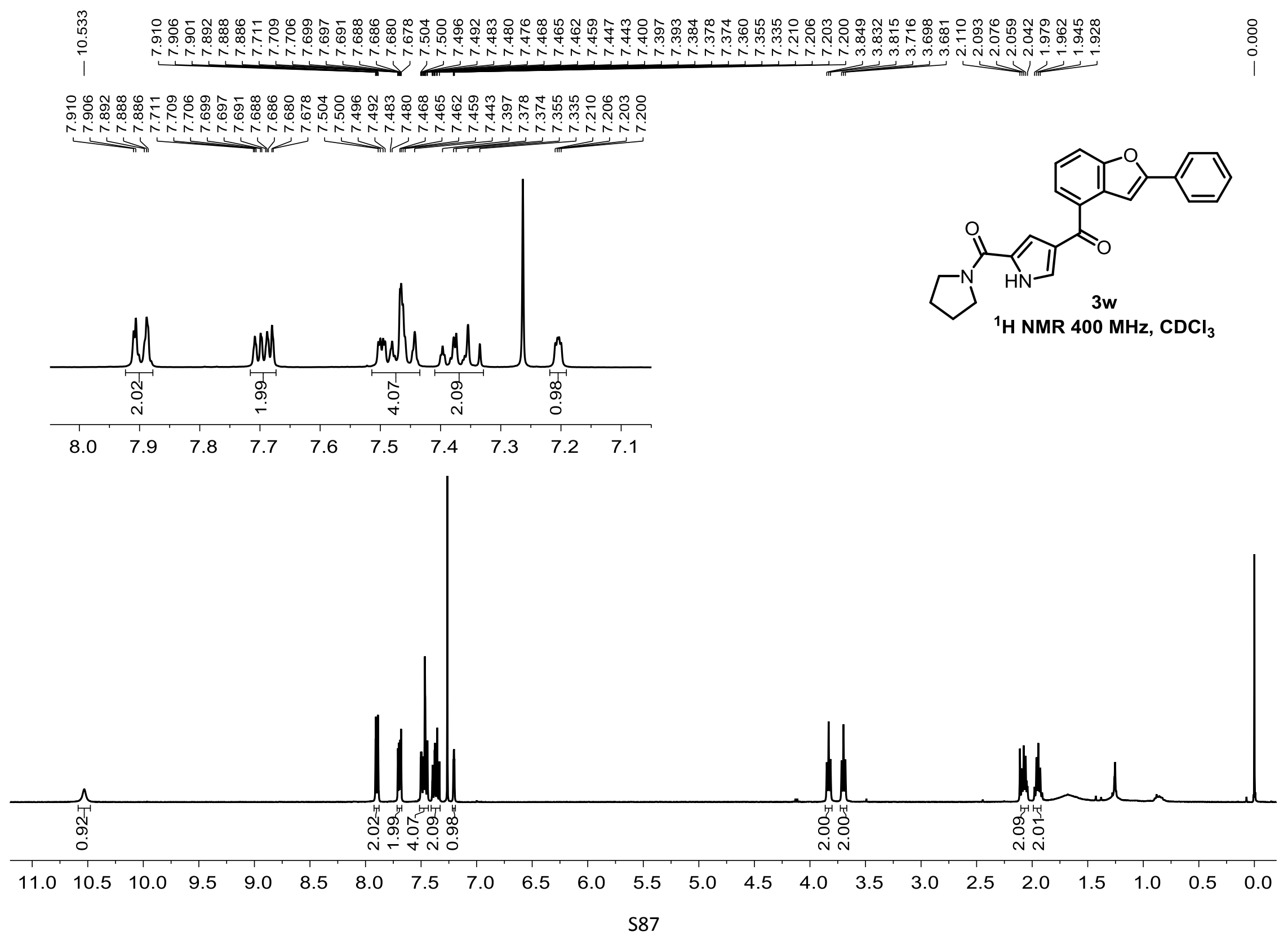




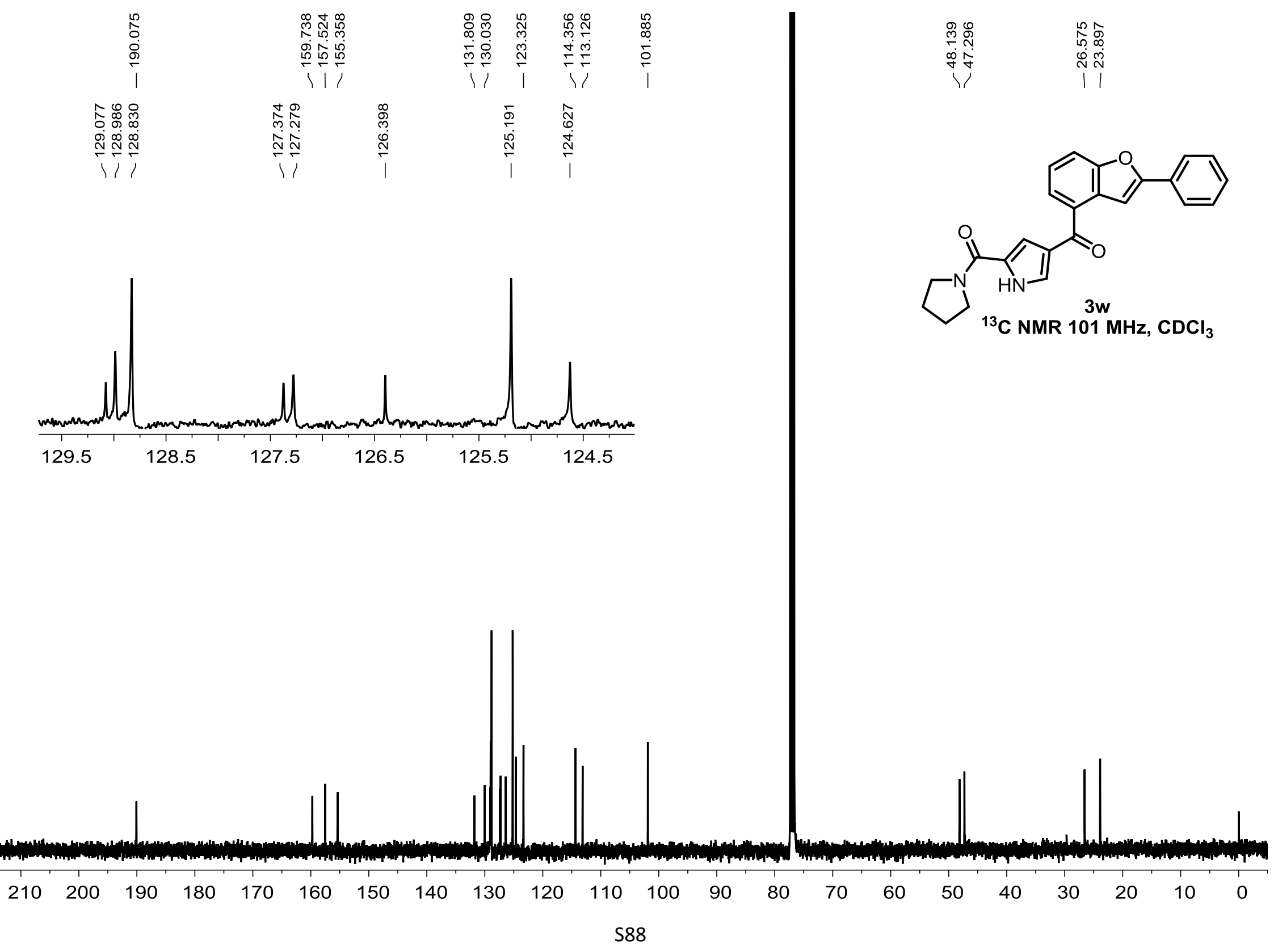




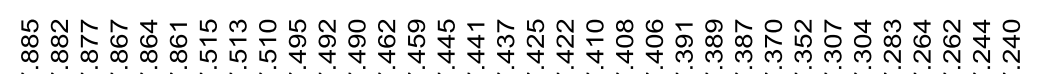

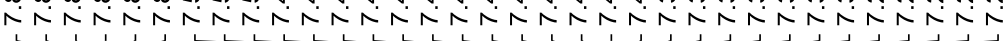
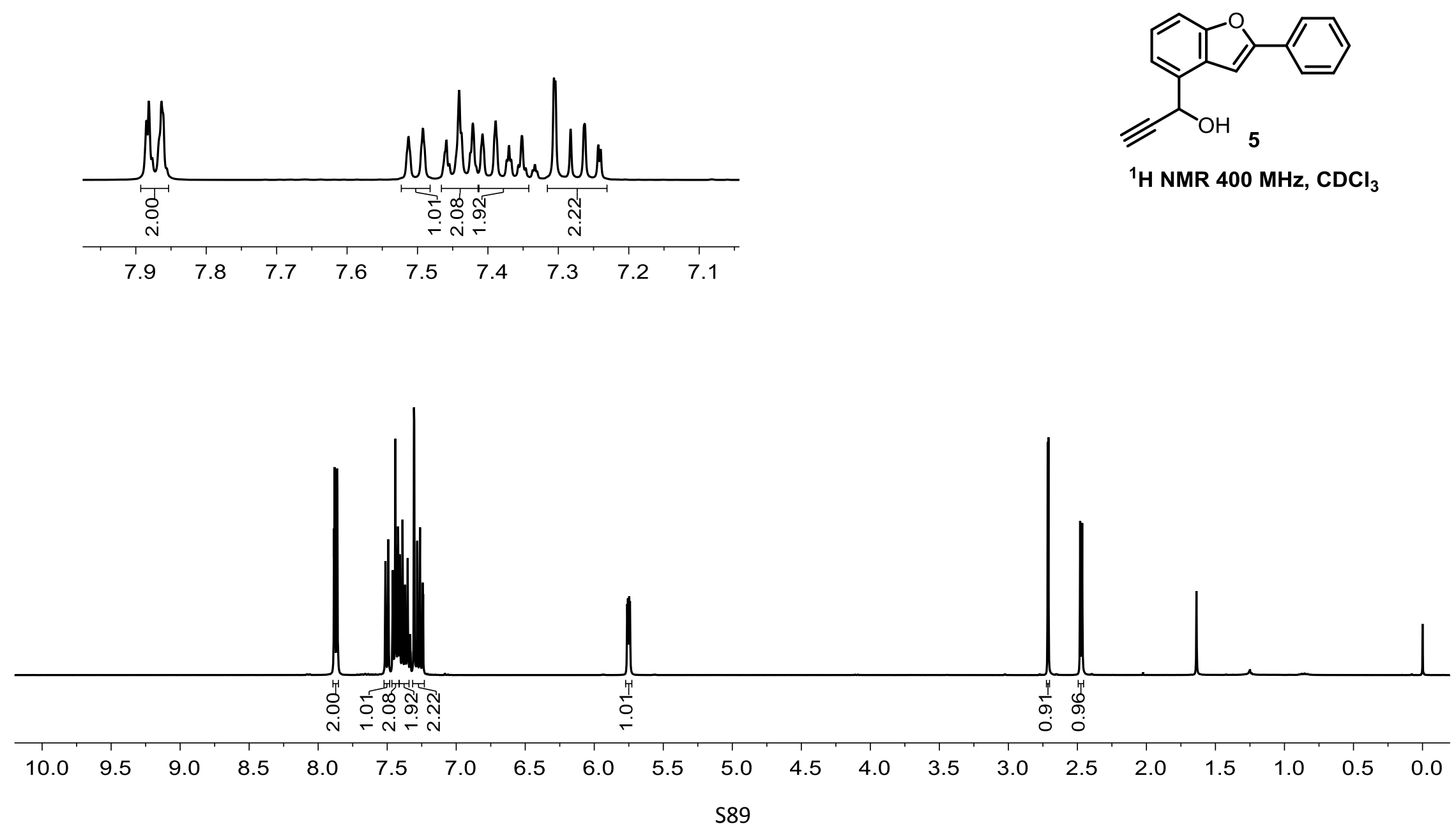


$$
\text { tent }
$$




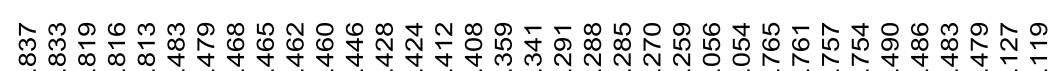

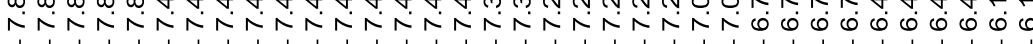

等
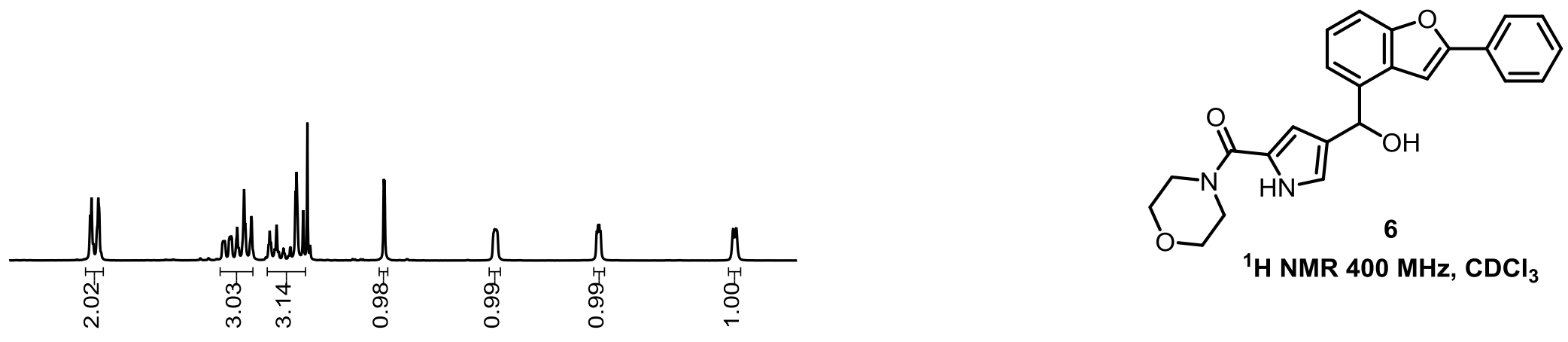

${ }^{1} \mathrm{H}$ NMR $400 \mathrm{MHz}, \mathrm{CDCl}_{3}$
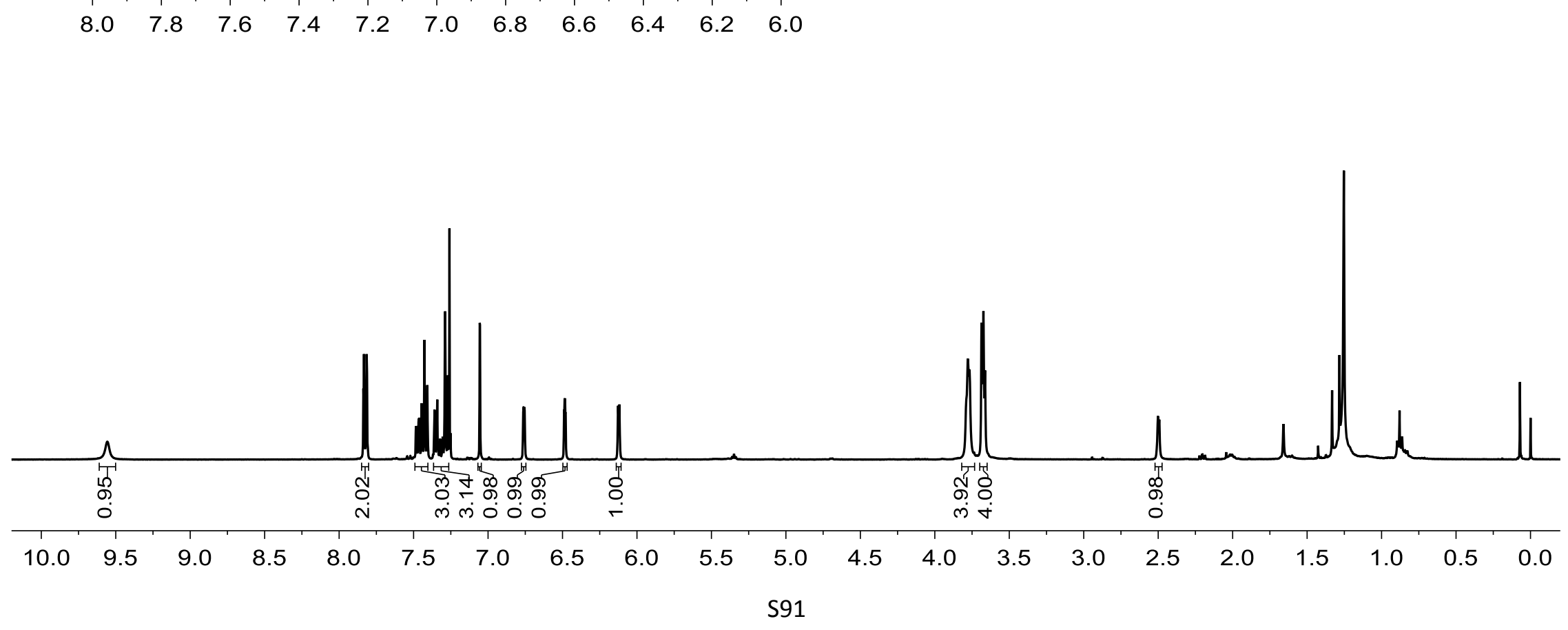


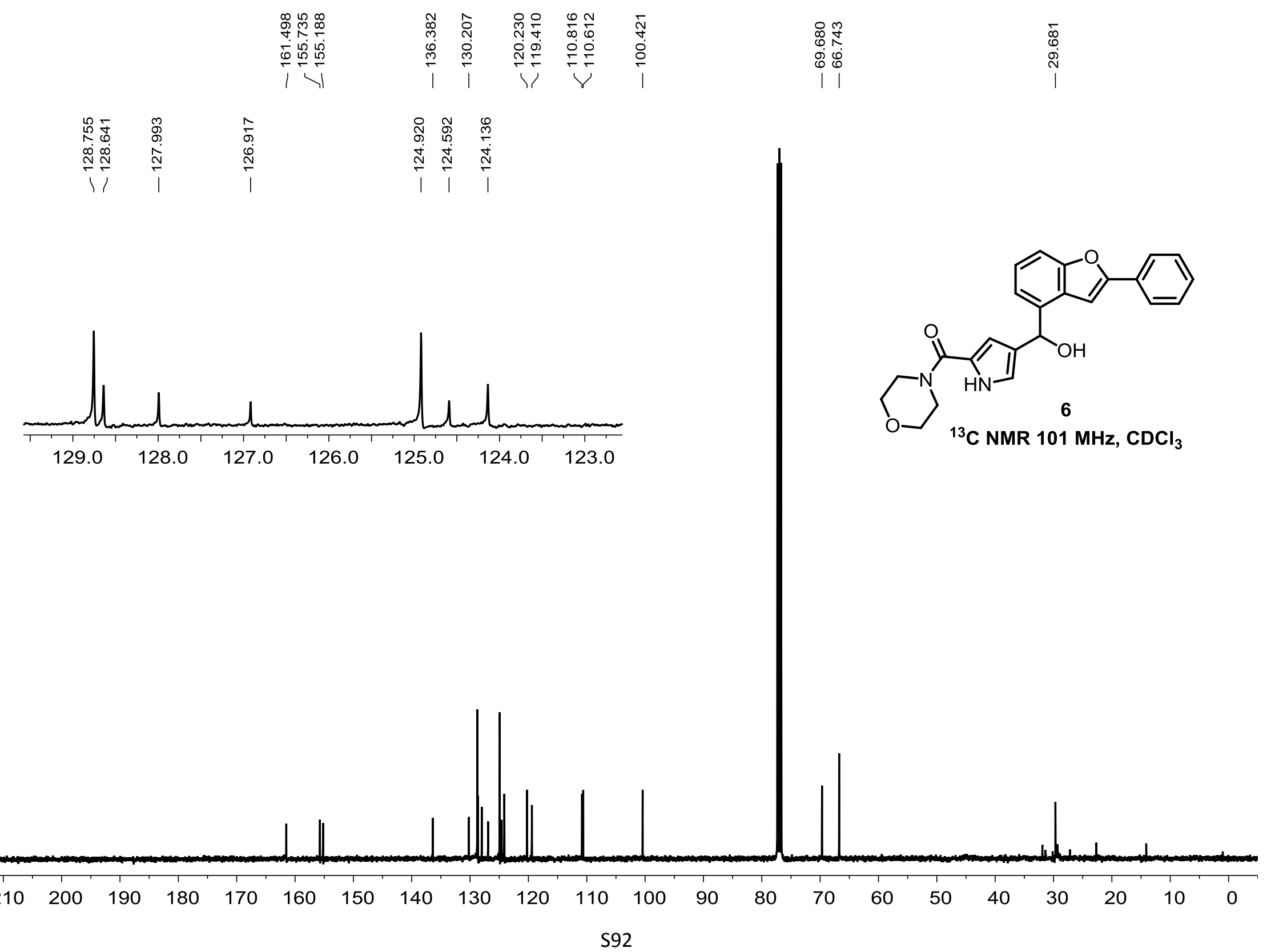




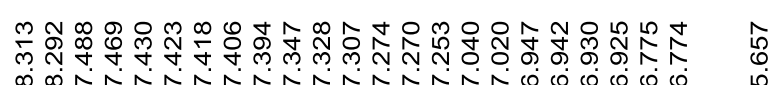

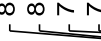

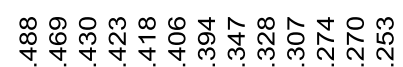

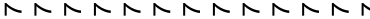

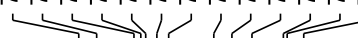

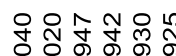

NNo000

114
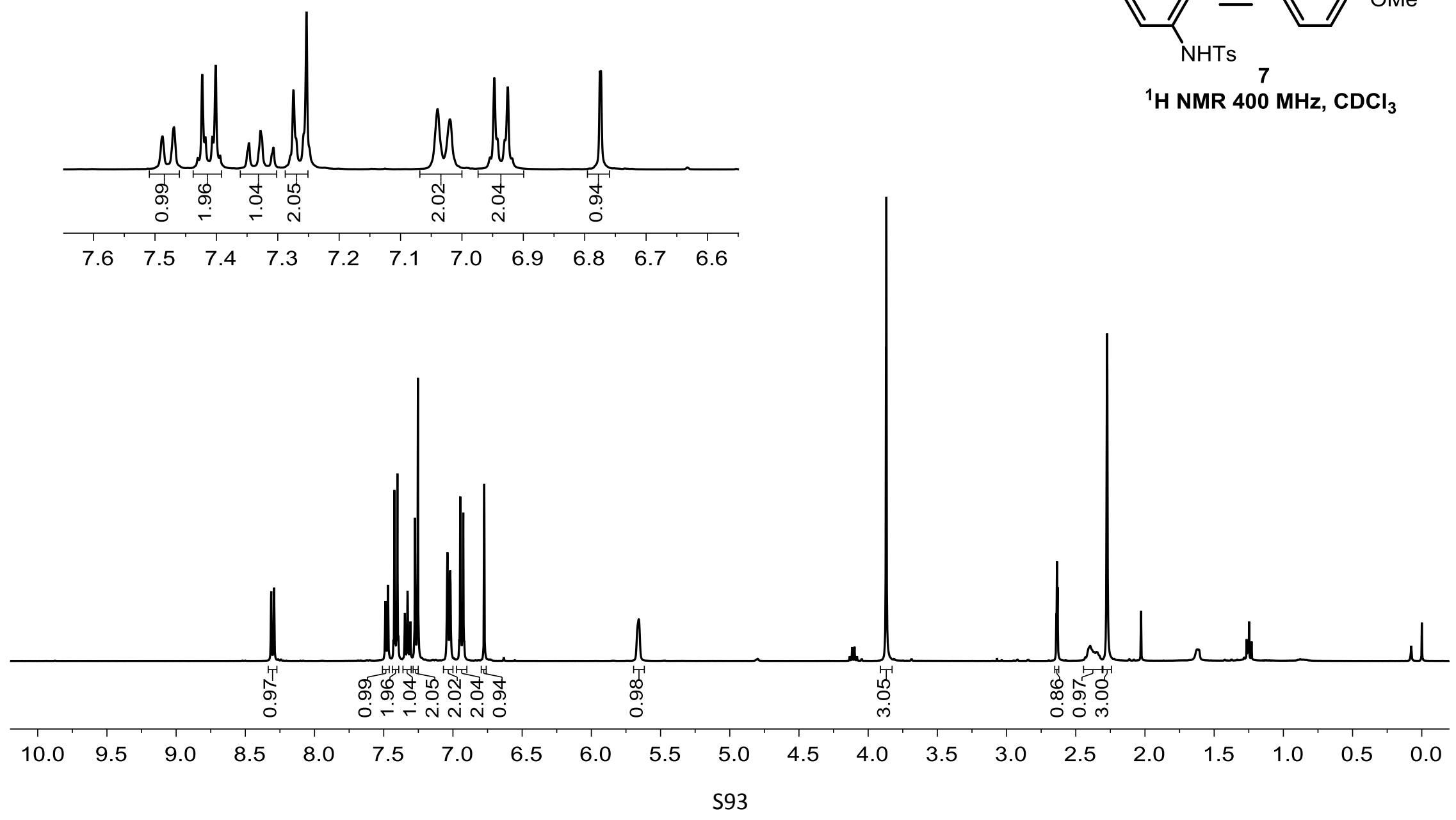

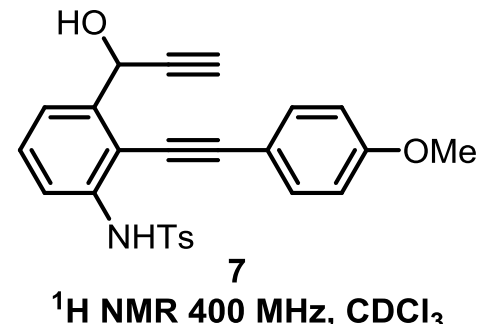

${ }^{1} \mathrm{H}$ NMR $400 \mathrm{MHz}, \mathrm{CDCl}_{3}$ 

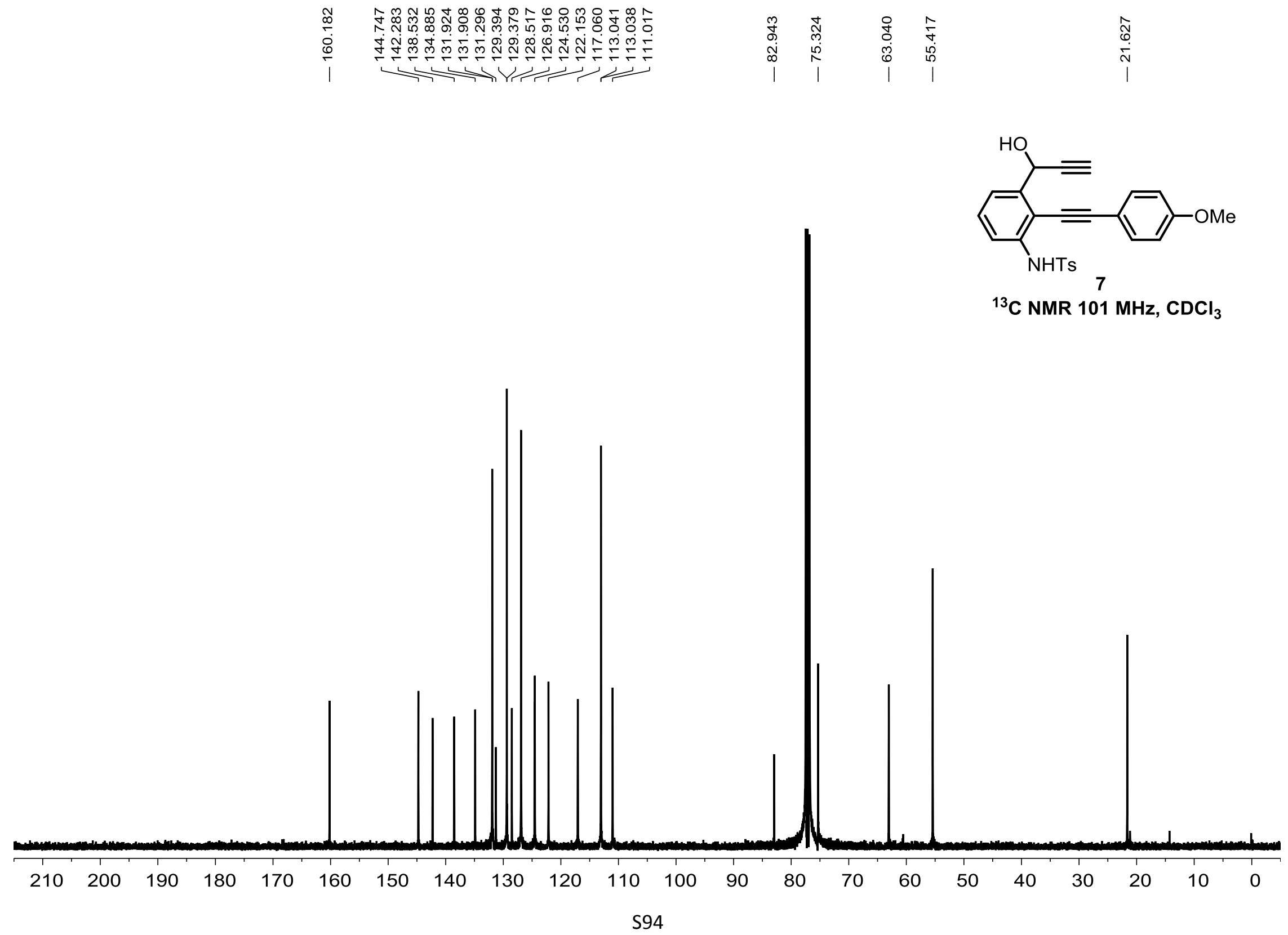


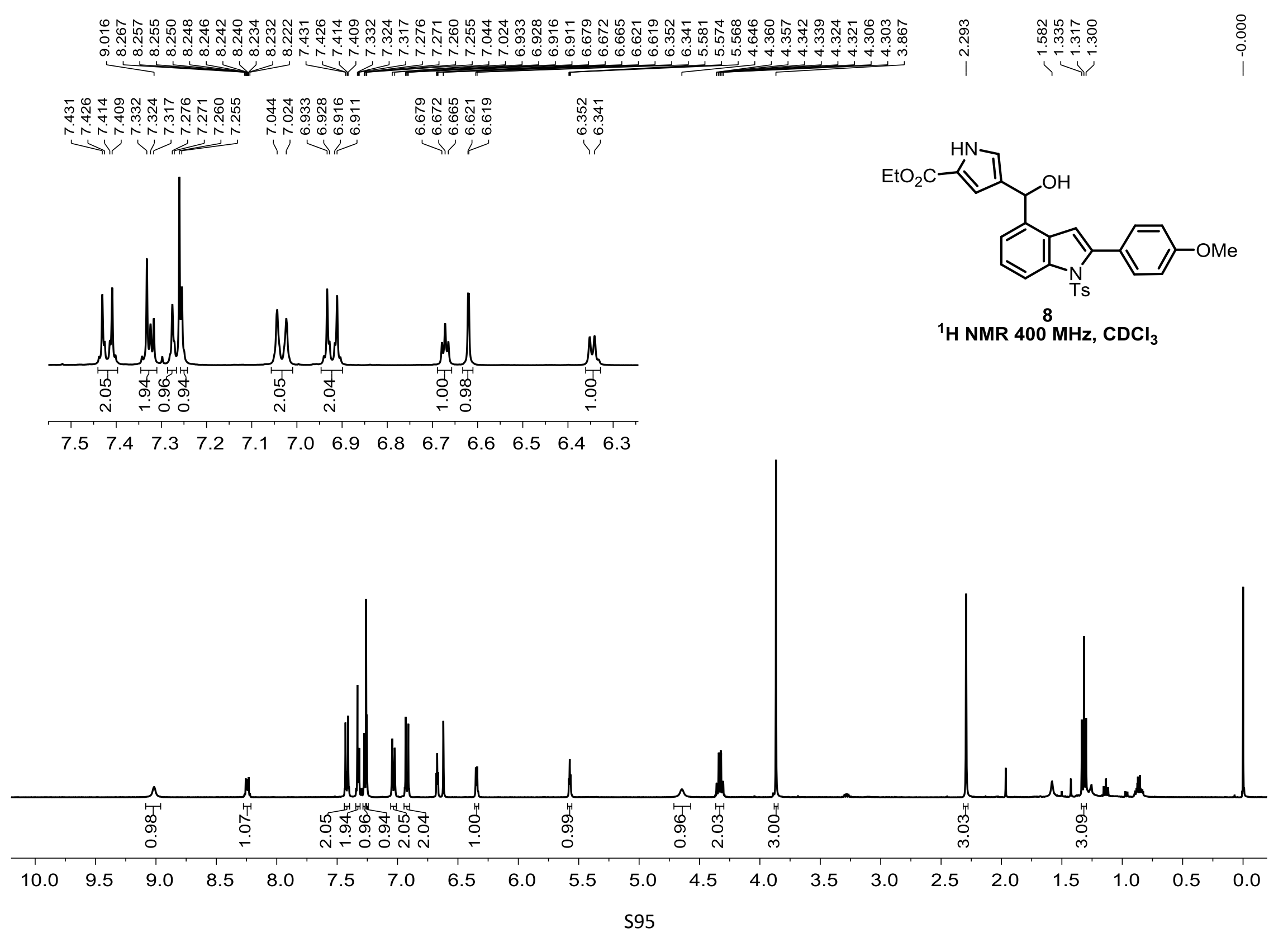




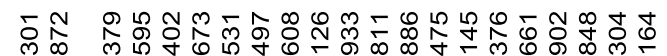

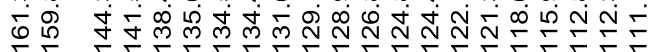

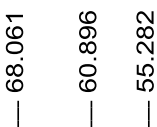

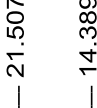

$\mathrm{EtO}_{2}$

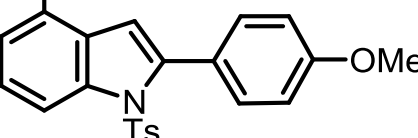

${ }^{13} \mathrm{C}$ NMR $101^{8} \mathrm{MHz}, \mathrm{CDCl}_{3}$

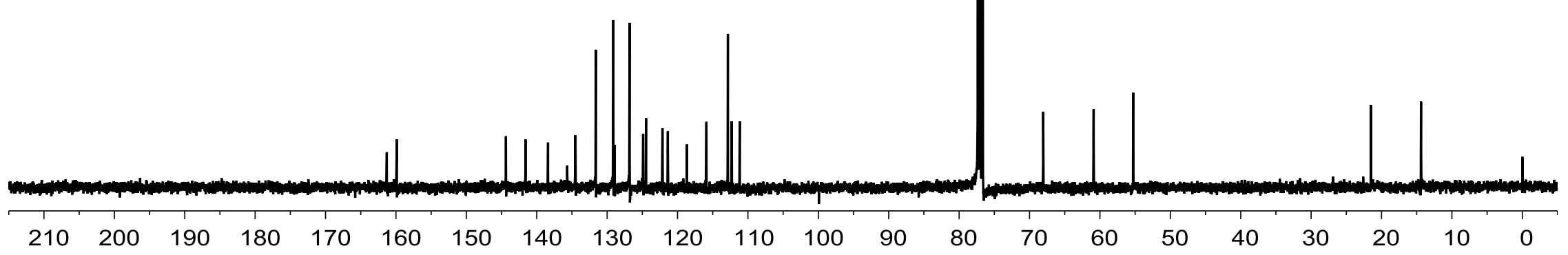

